\title{
CURVILINEAR VARIABLE STIFFNESS 3D PRINTING FOR IMPROVED MECHANICAL PERFORMANCE
}

\author{
By
}

Sadben Khan

Bachelor of Engineering, Ryerson University (2017)

\author{
A thesis \\ presented to Ryerson University \\ in partial fulfilment of the \\ requirements for the degree of \\ Master of Applied Science \\ in the program of \\ Aerospace Engineering
}

Toronto, Ontario, Canada, 2020

CSadben Khan, 2020 


\section{AUTHOR'S DECLARATION FOR ELECTRONIC SUBMISSION OF A THESIS}

I hereby declare that I am the sole author of this thesis. This is a true copy of the thesis, including any required final revisions, as accepted by my examiners.

I authorize Ryerson University to lend this thesis to other institutions or individuals for the purpose of scholarly research.

I further authorize Ryerson University to reproduce this thesis by photocopying or by other means, in total or in part, at the request of other institutions or individuals for the purpose of scholarly research.

I understand that my thesis may be made electronically available to the public. 


\title{
CURVILINEAR VARIABLE STIFFNESS 3D PRINTING FOR \\ IMPROVED MECHANICAL PERFORMANCE \\ Sadben Khan \\ Master of Applied Science, Aerospace Engineering, Ryerson University, Toronto (2020)
}

\begin{abstract}
Continuous Curvilinear Variable Stiffness (CCVS) is proposed as a novel design technique to generate Variable Stiffness design for improving the performance of composite panels featuring open-hole cut-outs. Compared to existing VS design techniques, CCVS steers the fibers around the cut-out without breaking at the holes using only a single design variable the geometry. The technique utilises a numerical method known as Source Panel method, which is typically utilised in the fluid dynamics world. Utilising this technique, the performance of an open hole ASTM D5766 coupon manufactured using Fused Filament Fabrication (FFF) was improved 16-38\% depending on the ratio of the hole to the width of the specimen. The technique was further improved on to allow for arbitrary geometries such as fuselage cut-outs. A fuselage cut-out case was examined, and it was shown that a CCVS design can improve the performance over a QuasiIsotropic design by $57 \%$. To validate CCVS, it is necessary to first manufacture and validate the part. This was done by developing a robotic 3D printing work-cell capable of 5 axis of material deposition of both thermoplastic and pre-impregnated carbon fiber. Finally, an in-process inspection technique was developed using a laser line scanner in the work-cell for the purposes of quality control.
\end{abstract}




\section{ACKNOWLEDGEMENTS}

I would like to firstly thank my supervisors Dr. Kazem Fayazbaksh and Dr. Zouheir Fawaz for supporting all the work presented in this thesis and provide mentorship throughout the way.

I would like to thank the members of the FRAMES lab at Ryerson for making the time spent in the lab enjoyable. I'd list you all, but there are too many of you...

I would like to thank Dr. Goetz Bramesfeld for offering his Applied Aerodynamics course and effectively teaching the material in a manner that was able to inspire the development of CCVS.

I would like to especially thank Dr. Hamid Ghaemi for going out of his way to support my work throughout my master's degree through his countless hours of technical consulting. 


\section{$\underline{\text { TABLE OF CONTENTS }}$}

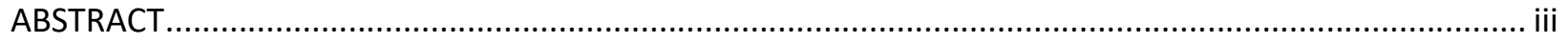

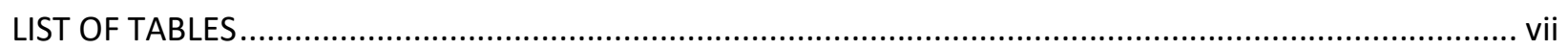

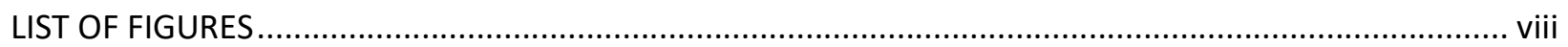

NOMENCLATURE

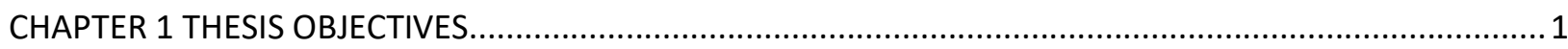

1.1. Continuous Curvilinear Variable Stiffness Design ............................................................... 1

1.2. Robot 3D printing of thermoplastics reinforced with Continuous Fiber composites................... 2

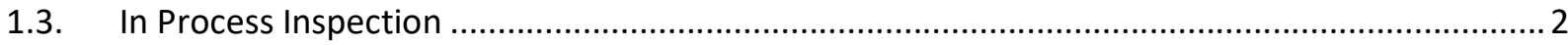

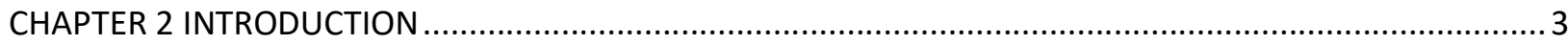

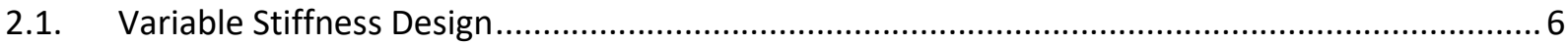

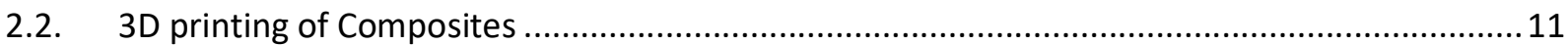

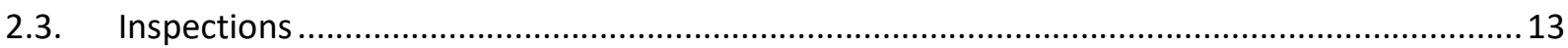

CHAPTER 3 CURVILINEAR CONTINUOUS VARIABLE STIFFNESS FOR IMPROVED OPEN HOLE TENSION.....18

3.1. Specimens geometry and stacking sequence ...................................................................... 19

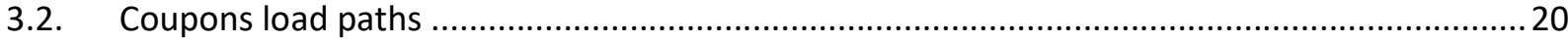

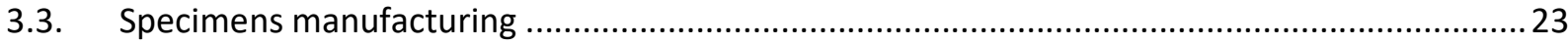

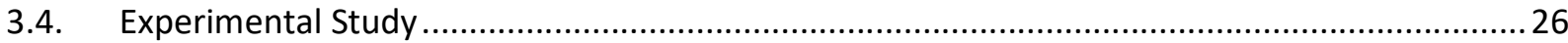

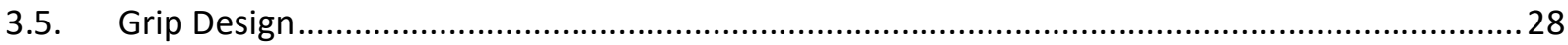

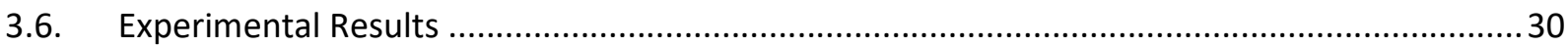

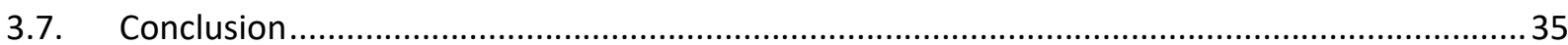

CHAPTER 4 CURVILINEAR VARIABLE STIFFNESS OF ARBITRARY CUT-OUT GEOMETRIES .........................37

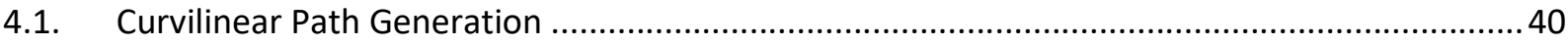

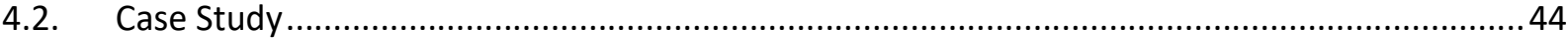

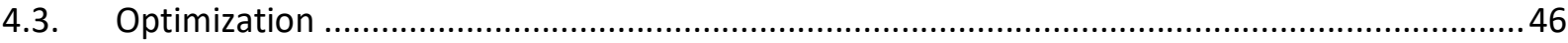

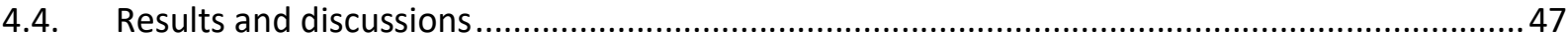

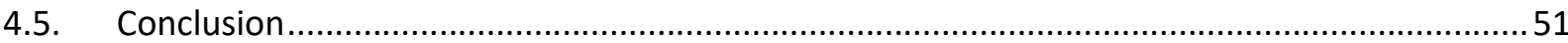

CHAPTER 5 INDUSTRIAL ROBOT 3D PRINTING USING FUSED FILAMENT FABRICATION (FFF) .................52

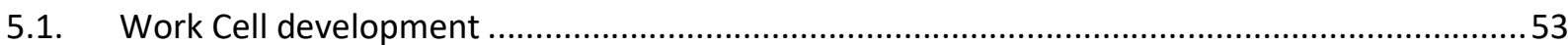

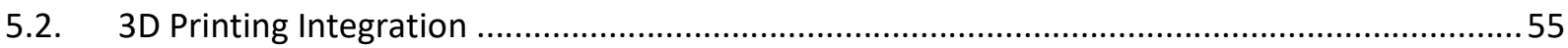

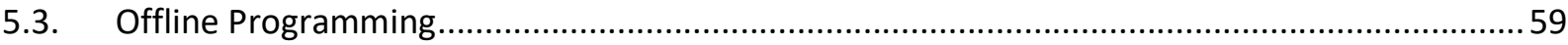

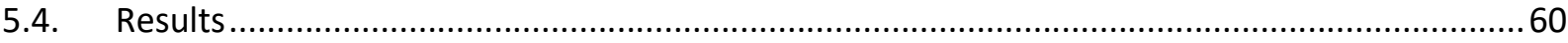

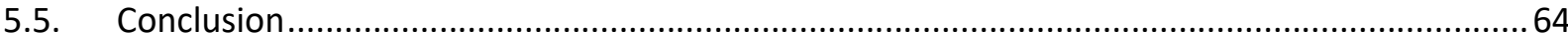




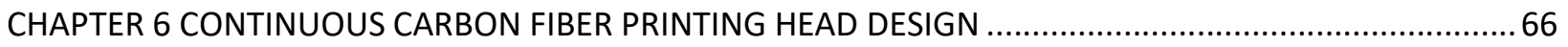

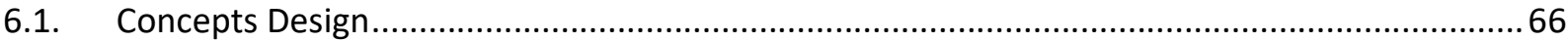

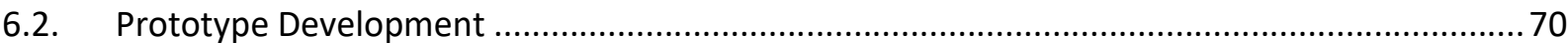

6.3. Continuous Carbon Fiber Head Design ................................................................................ 74

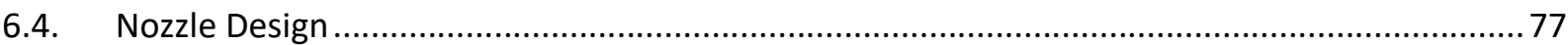

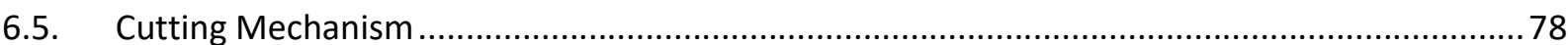

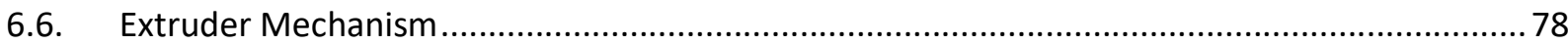

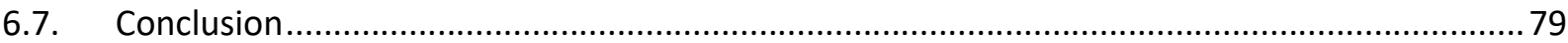

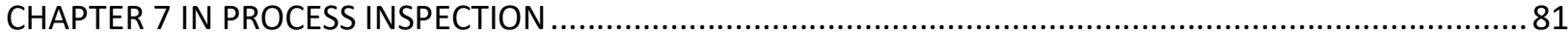

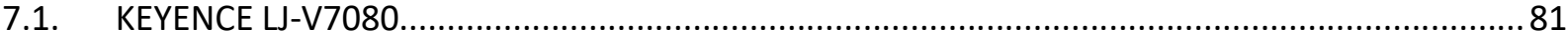

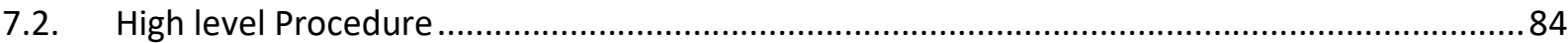

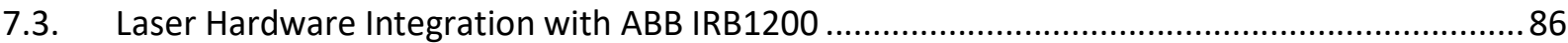

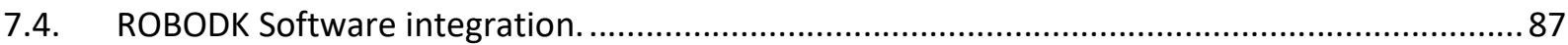

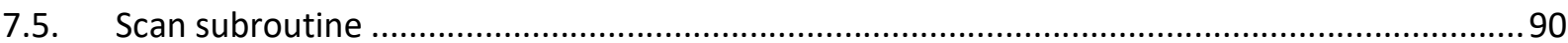

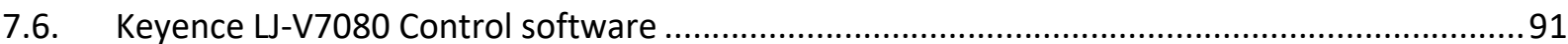

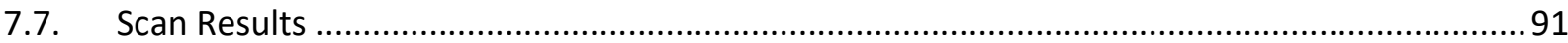

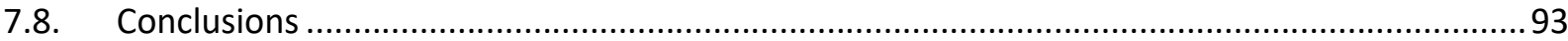

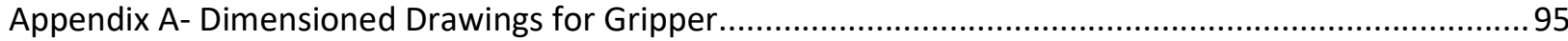

Appendix B- Dimensioned Drawings for 3D-Printing Head .............................................................. 100

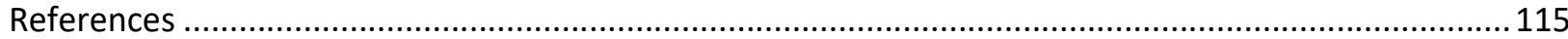




\section{LIST OF TABLES}

Table 3.1. Weight variation of manufactured VS and CS specimens..................................................27

Table 3.2. Ultimate strength and failure strain for open-hole tensile specimens ....................................34

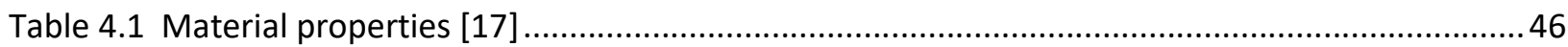




\section{LIST OF FIGURES}

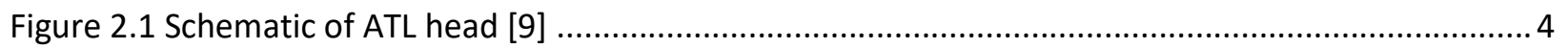

Figure 2.2 (A) MTORRES ATL machine , (B) Ingersoll ATL machine ....................................................... 5

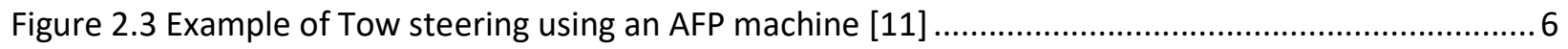

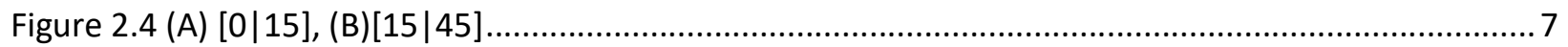

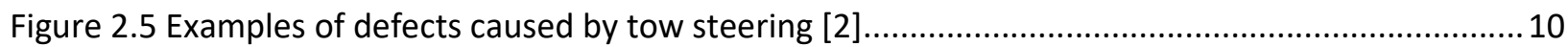

Figure 2.6 Steering capability demonstrated by $3 d$ printing of continuous carbon fibers [26] ................12

Figure 2.7 Out of plane 3D printing of continuous carbon fibers [32] .............................................. 13

Figure 2.8 Drone arm printed using continuous carbon fiber [31] .................................................... 13

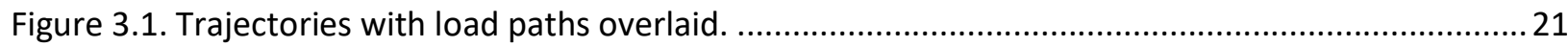

Figure 3.2 The load paths for open-hole tensile specimen with $w / D=4$ : (a) curvilinear variable stiffness,

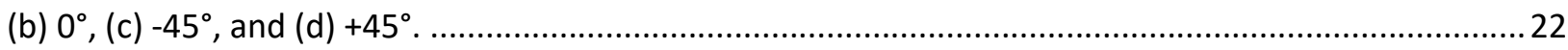

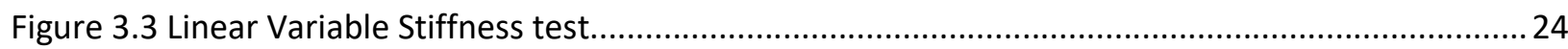

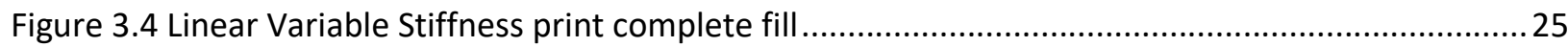

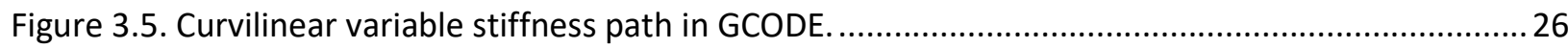

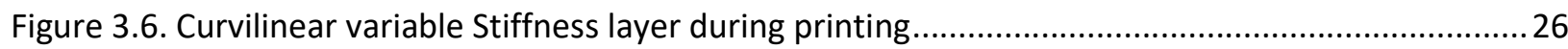

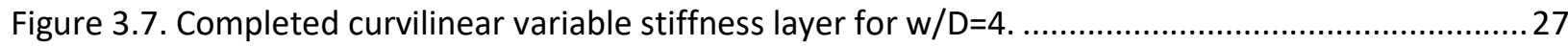

Figure 3.8. Open-hole tensile specimens with $w / D=4:$ (a) CS specimen and (b) VS specimen..................27

Figure 3.9 (A) Grips with Alignment Jig (B)grips with no jig............................................................2 29

Figure 3.10. The test set-up including a variable stiffness specimen $(w / D=4)$, the fixture, and the

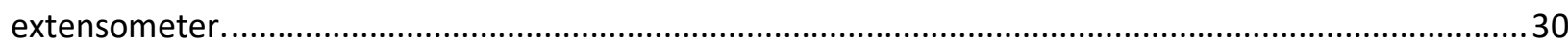

Figure 3.11. A variable stiffness specimen $(w / D=4)$ after failure (LGM mode) .................................... 31

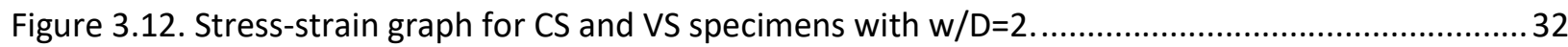

Figure 3.13. The cross sections of failed specimens ( $w / D=2)$ : (a) CS design; (b) VS design. ....................32

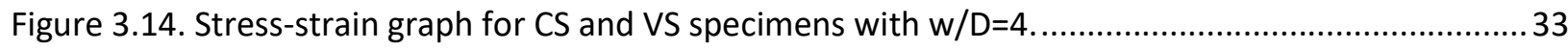

Figure 3.15. Mean strength for CS and VS designs for the two configurations ( $w / D=2$ and 4)...............35

Figure 4.1. Schematic of a single panel in the $X Q Y Q$ refrence frame ................................................37

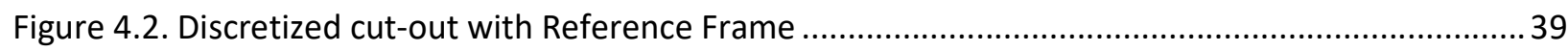

Figure 4.3. Discretized fuselage cut-out showing panels and collocation points................................... 41

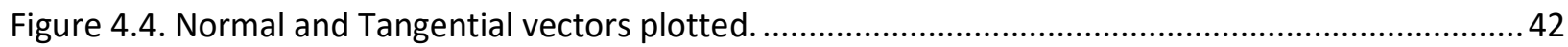

Figure 4.5. Examples of load path plotted at various angles: (a) $90^{\circ}$; (b) $45^{\circ}$; (c) $-45^{\circ}$; (d) $0^{\circ} \ldots \ldots \ldots \ldots \ldots . . . . . . .43$

Figure 4.6 Case study: (a) schematic of the plate with a central cut out; (b) applied loading (Pressure and external compressive force) where the exterior edges are simply supported and the internal opening

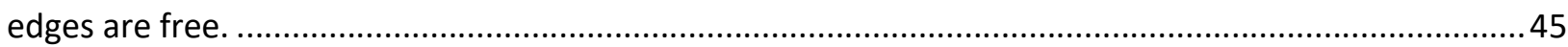

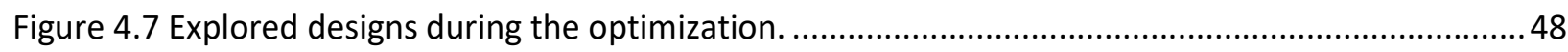

Figure 4.8 Envelope of stress distribution and failure index plots for the quasi-isotropic design. ............49

Figure 4.9 Envelope of stress distribution and failure index plots for the optimum CCVS design ...........49

Figure 4.10 Total displacement distribution plots (deformed shape with a factor of 100); (a) quasi-

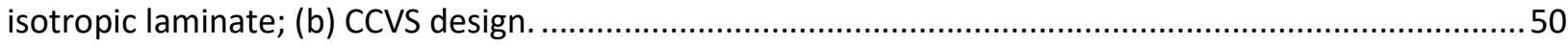

Figure 4.11 Layer number with the maximum failure index: (a) Quasi-isotropic laminate; and (b) CCVS

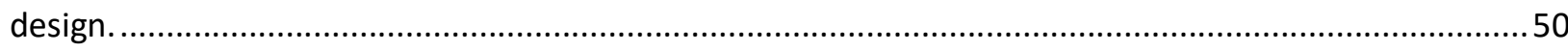


Figure 5.1 ABB IRB1200 Industrial Robot demonstrating degrees of freedom .................................... 53

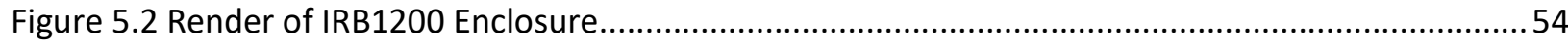

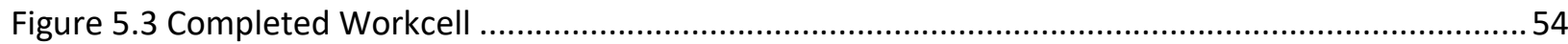

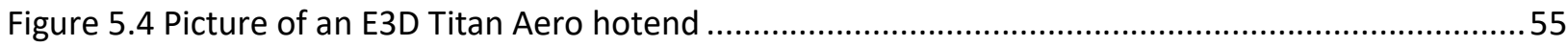

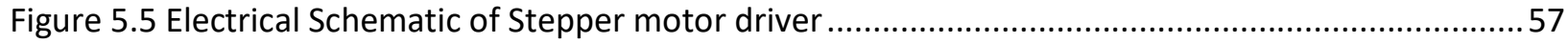

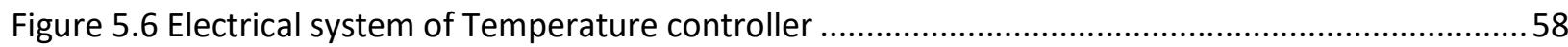

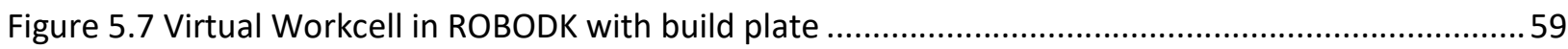

Figure 5.8 (A) TCP of Hot end visualised, (B) 3D model of Hotend assembly affixed to robot end effector

60

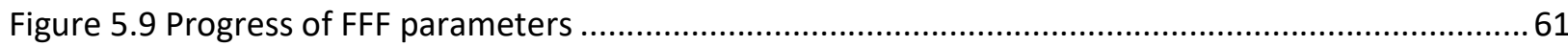

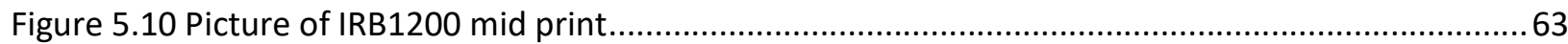

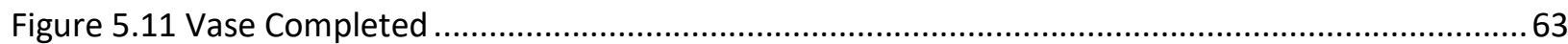

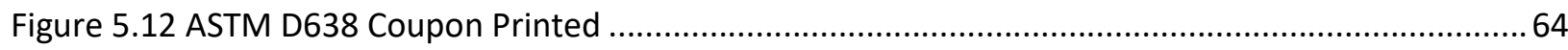

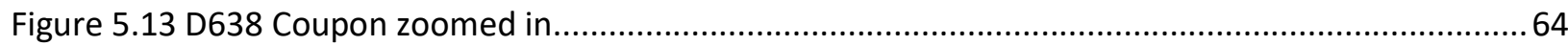

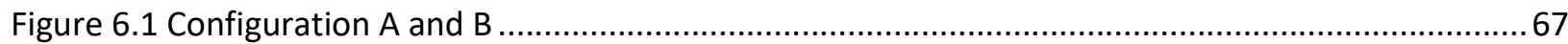

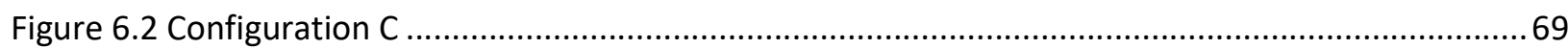

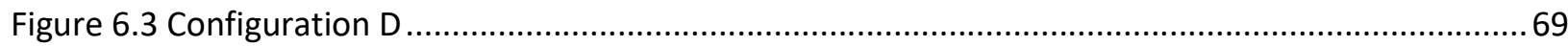

Figure 6.4 Micrograph of Markforged carbon fiber prepreg ............................................................. 70

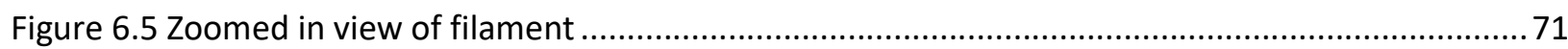

Figure 6.6 (A) CAD model of continuous carbon fiber hot end, (B) Integrated onto IRB1200 ..................72

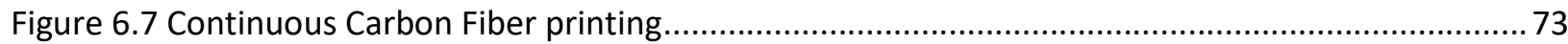

Figure 6.8 ASTM D3039 Tensile test coupons manufactured with Markforged Continuous Carbon Fiber73

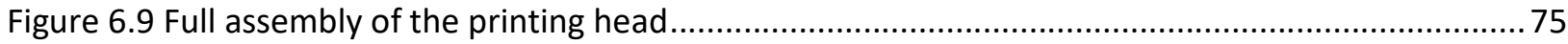

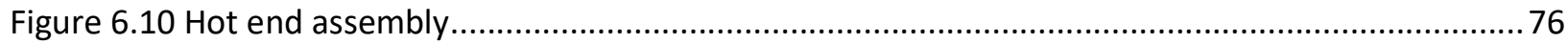

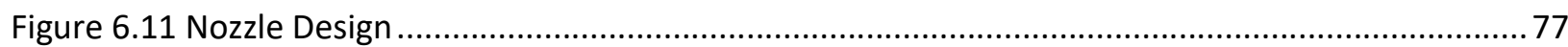

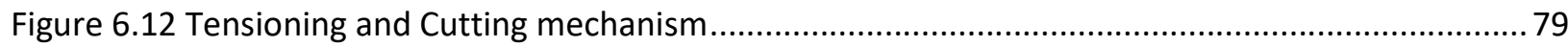

Figure 7.1 (A) Reference 3D printed Part, (B) Keyence L-V7080 ,(C) Micro Epsilon, (D) Creaform ........... 82

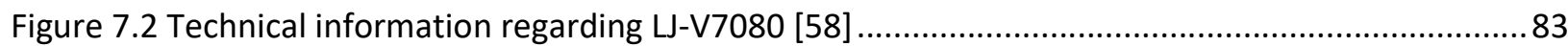

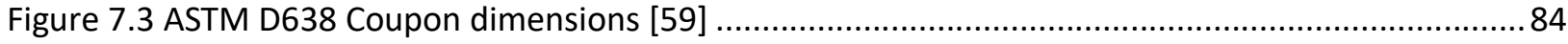

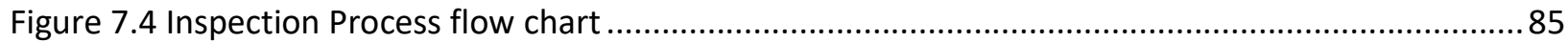

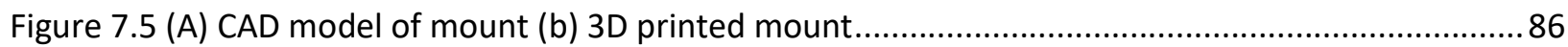

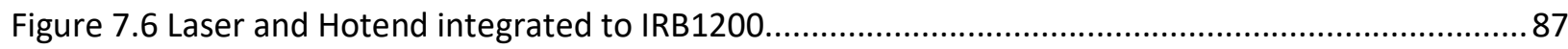

Figure 7.7 CAD Model of Scanner and Hotend integrated to IRB1200 Virtual Model .............................88

Figure 7.8(A) TCP for FFF nozzle, (B) TCP for LJ-V7080 Scanner ........................................................89

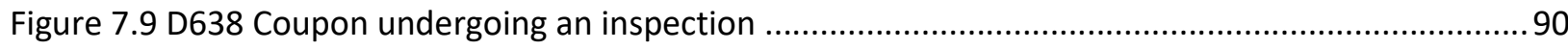

Figure 7.10 (A) Photograph of D638 Coupon (B) Coupon scanned with LJ-7080 ................................ 92

Figure 7.11 Zoomed in view of coupon scan highlighting defects with false colour ................................92

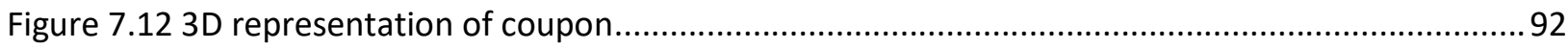

Figure 7.13 (A) 0 Degrees (B)90 Degrees (C) 45 Degrees (D) -45 degrees ..........................................93 


\section{NOMENCLATURE}

$\phi$-Velocity Potential

$r$-radius

$\theta$ - Angle

$R$ - Hole Radius

$r$ - Distance from hole centre

$V_{r}$ - Radial Velocity (Polar coordinate system)

$V_{\theta}$-Tangential Velocity (Polar coordinate system)

$P$-Abritary point in $(x, y)$ coordinate system

$X_{Q^{-}}$Local $x$-axis in transformed coordinate system

$Y_{Q}$ - Local $y$-axis in transformed coordinate system

$\Delta s$ - Length of source panel

$Q$-Source Strength

$\xi$-Arbitrary distance along source panel

$v_{Y_{Q}}-Y$ component of the velocity oriented in the panel reference frame

$v_{X_{Q}}-\mathrm{X}$ component of the velocity oriented in the panel reference frame

$\phi_{P Q}$ - Velocity in panel coordinates

$\overrightarrow{v_{x y}}$-Transformed velocities to global cartesian coordinates.

$\widehat{n_{l}}-$ Normal Vector

$\widehat{t}_{l}-$ Tangential Vector 


\section{CHAPTER 1 THESIS OBJECTIVES}

The objective of this thesis is to develop a methodology for improving the performance of structures with large structural discontinuities such as fuselage cut-outs as well as the preliminary method by which this design will be manufactured and inspected. Therefore, the thesis is broken down into three major sections: Development of continuous curvilinear variable stiffness design(CCVS), Design of a continuous carbon fiber robotic $3 \mathrm{~d}$ printing work-cell, and finally an In-Process inspection of 3D printed parts using a laser line scanner.

\subsection{Continuous Curvilinear Variable Stiffness Design}

Many of the works that are described in Chapter 2. investigated large cut-outs and discontinuities in composite structures and used Variable Stiffness (VS) design in an attempt to improve the load carrying capacity of the part. A common trend in the results of many of these studies were that the fiber paths around the cut-out tended to steer around the large discontinuity similarly to fluid flow. Despite the fibers tending to steer around the cut-out or discontinuity, the fibers were not continuous and would still break at the edge of the cut-out.

Continuous Curvilinear Variable Stiffness (CCVS) design is a novel technique that was developed for continuously steering fibers around cut-outs and discontinuities using a Potential Equation method. In this thesis preliminary investigations towards the performance of CCVS and initial tensile tests are described in Chapter 3with the performance improvement of a circular cut-out undergoing a tension load. Chapter 4 further improves on CCVS with applications of the design technique toward arbitrary geometry, in this case a fuselage cut-out, using an approximation technique known as Source Panel method. 


\subsection{Robot 3D printing of thermoplastics reinforced with Continuous Fiber composites}

For validating the CCVS design a robot work cell capable of 3D printing with continuous carbon fibers was developed. A design technique can improve the performance of a structure significantly, but it is meaningless if it is not feasible to manufacture. In Chapter 2, the works described showed the incredible versatility of continuous carbon fiber 3D printing but commonly lacked the function of cutting the fiber between each pass and the ability for compaction of each deposited layer. Therefore, a continuous carbon fiber 3D printing cell was developed with the capability of cutting the fiber at the end of the pass and compacting the fiber. The development

of this system is described in Chapter 5, with initial prototyping done through FFF using a thermoplastic and the final design and preliminary results described in the following section in Chapter 6.

\subsection{In Process Inspection}

Previous research studies used NDT techniques for quality inspection of 3D printed parts after fabrication or a qualitative method for determining defects. For high performance materials, e.g. PEEK reinforced with high modulus carbon fiber, an in-process inspection would be critical. A real-time inspection can detect defects during manufacturing and stop the print or give indications for rectifying them. An in-process inspection is proposed to accurately quantify each layer in an FFF process and is implemented using a Keyence L-V7080 laser line scanner. Compared to other processes described in Chapter2, the laser line scanner method generates a 3D point cloud of each layer that can then be inspected for defects or correlated onto a Finite Element model. This process is detailed in the final chapter, Chapter7. 


\section{CHAPTER 2 INTRODUCTION}

Composites feature very high strength to weight ratios and have been featured as the material of choice for primary structures in modern aircraft design. Composites in aircraft are typically a Polymer Matrix Composite (PMC) which consists of carbon fibers bonded together via a polymeric resin. Compared to similar structures constructed from traditional metal alloys, composite structures feature greater fatigue life and lesser complexity due to the reduced requirements for joints. An interesting property of composite laminates is the anisotropic properties that can be induced in a structure based on the ply angles in the layup. As a result composite structures can be tailored during the manufacturing to improve the strength to weight of the structure or add additional properties due to the directional stiffness of the laminate. For example, a wingbox can be tailored such that the primary direction of the fibers in the laminate is in the spanwise direction, thereby increasing stiffness when loads are applied in that direction [1].

Laminated composites have traditionally been manufactured by skilled technicians using a cumbersome hand lay-up and vacuum bagging process. This is less than ideal for large structures such as a wing skin and fuselages, which would require a large labour force to complete on schedule. Hand lay-up is also incredibly labour intensive and the defects induced by human error in the placement of the plies introduce process variabilities that may induce undesirable properties in the final part. For several decades, the difficulty in producing large structures reduced the adoption of composite materials to only a fraction of the structural mass of an aircraft. The introduction of Computer Numerical Control (CNC) allowed for a degree of automation through filament winding, but automated fabrication of composite structures was truly considered viable only after the introduction of Automated Tape Laying (ATL).

ATL is a process that deposits a strip of material, usually a prepreg carbon fiber unidirectional tape, along a predefined path [2]. ATL can be considered a form of Additive Manufacturing, as the material is built up by adding material, rather than subtracting from a stock. The ATL technology first emerged commercially in the early 1980's as the demand for more 
fuel-efficient aircraft grew due to the high price of oil in that era [3]. The earliest known development of ATL was by Chitwood and Howeth [4] in 1971, who had a patent assigned for a composite tape-laminating machine that was based on CNC. A few years later Golds-worthy applied for a patent for a system that would be capable of depositing tape onto complex curved surfaces. At this point in the mid 1970's, many ATL machines were designed and built in-house by Aerospace Corporations or Academic research institutions. Due to the commercial need for the technology, ATL became a generic process. Initial development for the commercial market was independently developed by Eaton [5] and Saveriano [6] in 1984. Both systems had limitations on geometric complexity and scalability in the commercial space. To address these concerns, Cincinatti-Milacron developed an ATL machine using an ultrasonic compaction system, but with limitations on speed and material deposition [7]. The modern ATL machine design from which most machines today are patterned after was introduced in 1986 by Meier, which featured a force controlled compaction system as opposed to the previous ultrasonic compaction systems [8] [2].

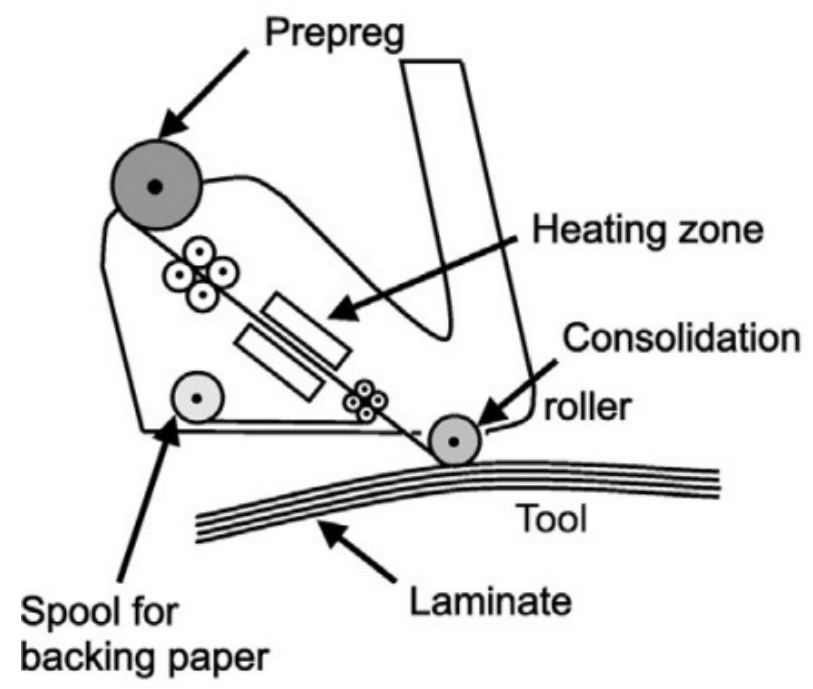

Figure 2.1 Schematic of ATL head [9] 


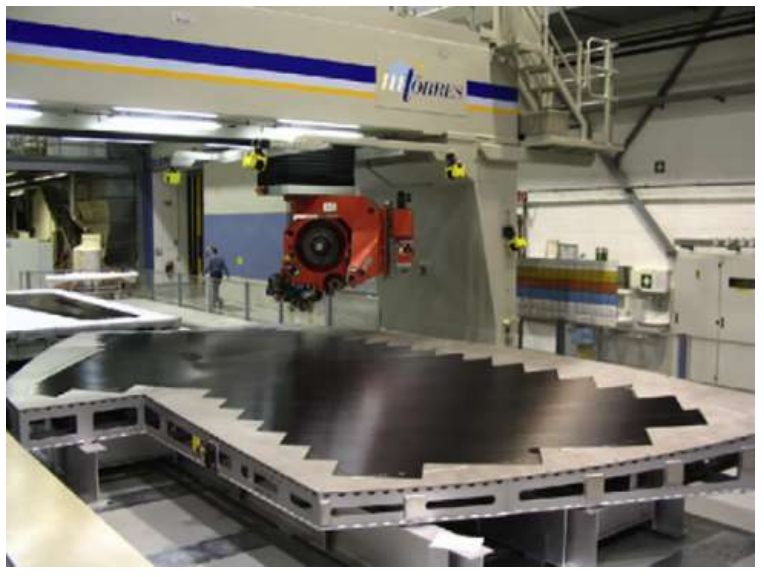

(A)

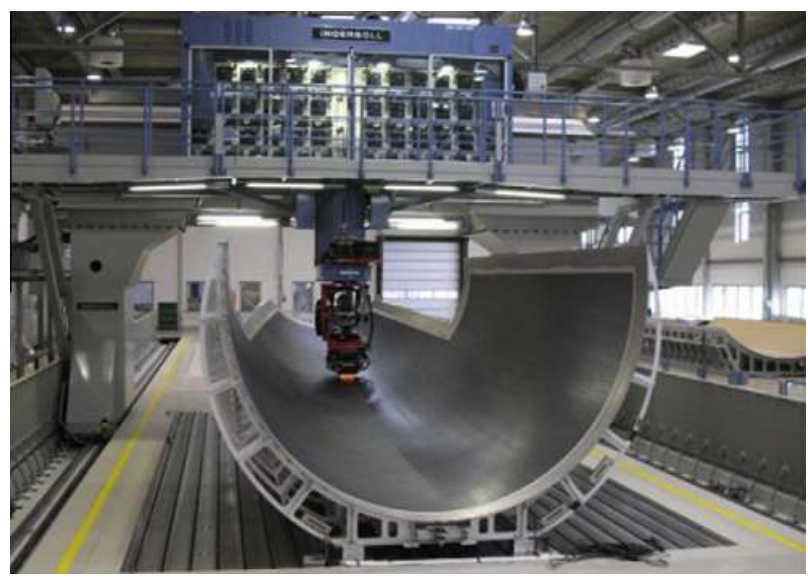

(B)

Figure 2.2 (A) MTORRES ATL machine, (B) Ingersoll ATL machine

ATL systems are typically gantry based with more advanced systems featuring end effectors capable of multi-axis deposition. The key benefit of an ATL systems is the rapid deposition of material with great precision and repeatability. The laydown rate compared to human technicians is immeasurably faster, thus allowing for the production of large composite structures. ATL machines have a few limitations, particularly in terms of size. Most ATL machines are very large gantries designed for manufacturing of large aircraft components such as the aforementioned fuselage sections and wing skins. Due to the large form factor and relatively large tow width, the possible curvatures on the part are limited. Furthermore, the gantry system limits the flexibility of the system, thereby limiting possible orientations the end effector is able to reach on the tooling. A derivative of the ATL process known as Automated Fiber Placement (AFP) addresses such issues.

Typical AFP systems are affixed to a 6 axis industrial robot. Using an industrial robot compared to a custom designed gantry reduces the engineering and manufacturing cost while offering a more flexible system that can be tailored according to the end users needs. The systems can be modified for a variety of structural sizes and complexity by adding OEM specific parts such as linear rails or additional robots. The use of a commercial off the shelf robot come at a cost however, as the 6-axis robot will not have the same accuracy and speed as a gantry system. This is a result of the motion being interpolated through the joints of the robot and its relatively light weight compared to an ATL machine. In an ATL the mass of the end effector is 
negligible to the overall mass of the gantry, so issues related to the flexibility of the machine and overcoming inertia are typically not a concern.

Due to the smaller size of the machine and narrower strips of tape, AFP machines allow for production of far more complex parts than what would be achievable with an ATL machine. One interesting capability of narrow tapes is the ability to steer the tow along a radius. By doing so, the direction of the fiber follows a non-linear path along the part surface. By manipulating the fiber path using this technique it is possible to vary the stiffness in a laminate, in a process called Variable stiffness (VS). In a VS design, fiber paths could follow a curvilinear path and therefore a changing orientation within each ply, thereby changing the stiffness locally [10]. The introduction of variable stiffness in a laminate design creates novel characteristics and the potential of significantly improving its load carrying capacity in different applications.

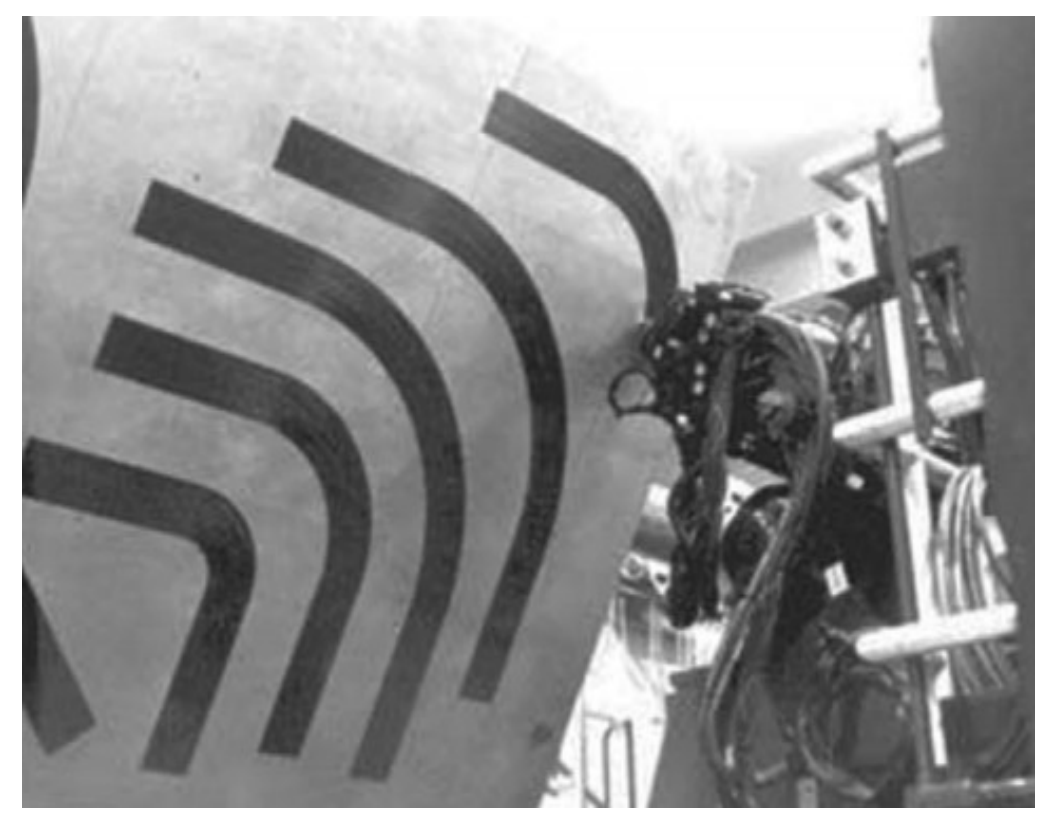

Figure 2.3 Example of Tow steering using an AFP machine [11]

\subsection{Variable Stiffness Design}

The concept of variable stiffness was first formally published by Gurdal and Olmedo [12], who outlined an analytical method for creating variable stiffness fiber orientations as well as a 
closed form solution derived from classic laminate theory. The angle varied linearly in the $x$ direction according to $\left(2.1\right.$, where $\theta_{1}$ is the initial angle at the center of the panel and $\theta_{0}$ is the angle and the end of a panel with length $a$.

$$
\theta(x)=\frac{2\left(\theta_{1}-\theta_{0}\right)}{a} x+T_{0}
$$

Using Eq(2.1), Figure 2.4 was generated using a MATLAB code written by the author of this thesis for visualising the VS panels.

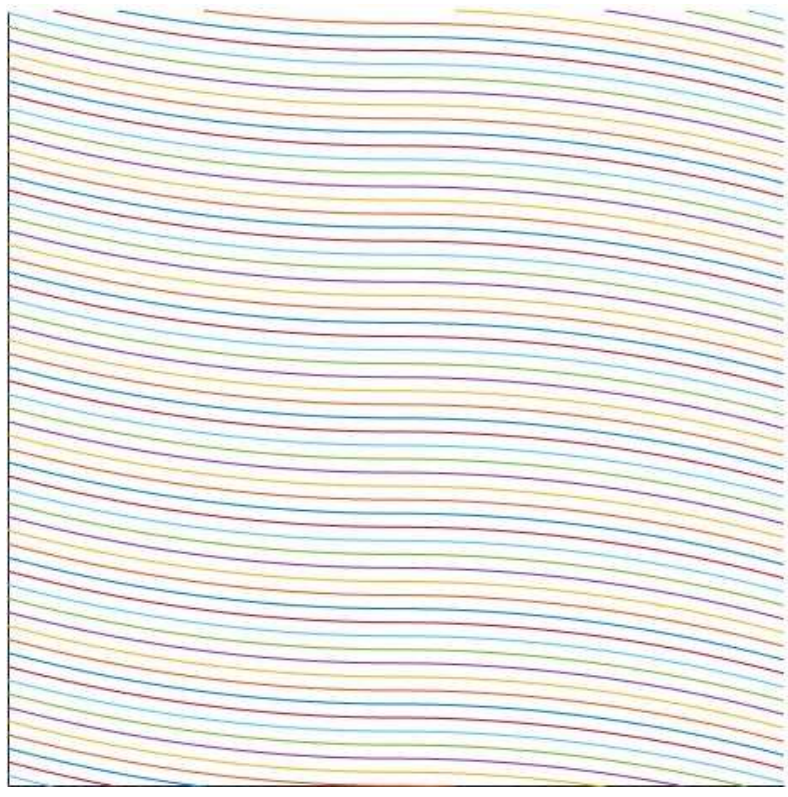

(A)

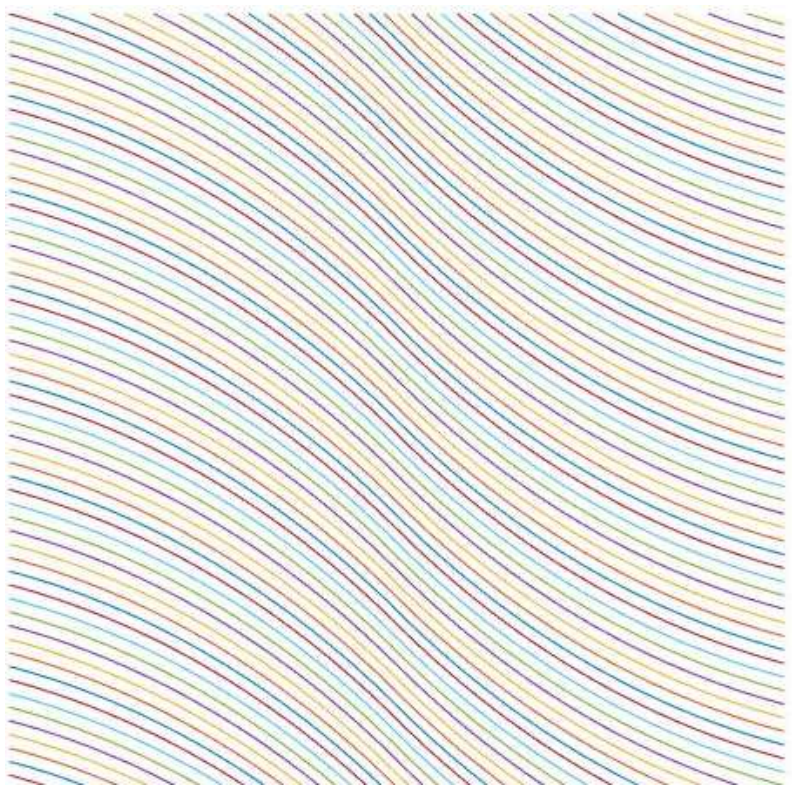

(B)

Figure $2.4(A)[0 / 15],(B)[15 / 45]$

The elastic behaviour of VS panels is unique compared to Constant Stiffness (CS) in that the mechanical properties will vary depending on the position across the panel. Such a property is useful in that it potentially distributes stresses around a structure as well as potentially generating highly optimised designs. In-plane uniaxial compression was investigated by Waldhard et al [13], where the VS panel was modeled using a Finite Element Method (FEM). Two methods for generating the paths were examined, the Shifted fiber method and the parallel fiber method. The shifted fiber method utilised a reference fiber path that is shifted along the $y$ axis of the panel. This results in fibers that become either bunched or spread out when the difference 
in fiber angles is large as seen in Figure 2.4. The parallel fiber angle method uses a optimisation technique to vary of each fiber according to the reference path such that they are parallel at each point along the panel. The laminates that were examined included a $[ \pm 45]_{9 s}$ straight fiber case, a $\left[ \pm 45_{2} \quad 90 \pm\langle 30 \mid 75\rangle_{7}\right]_{s}$ shifted fiber case and $\left[ \pm 45_{2} \quad 90 \pm\langle 45 \mid 60\rangle_{7}\right]_{s}$ parallel fiber case. The results indicated that the shifted fiber improved the buckling load compared to the straight fiber case by $36 \%$ while the parallel fiber improved the buckling load by $7 \%$. The improvement in the shifted design was thought to have arisen from the redistribution of the loading towards the edges of the plate. The parallel fibers were found not to have as much of an improvement due to restrictions in the angle variation for the reference path.

A major concern in aircraft fuselage design is the inclusion of windows and cut-outs, which introduce large discontinuities in the structure. As most of the cabin internal pressure load is carried by the fuselage skin, it is necessary to optimally design cut-out areas to reduce stress concentrations. Typically, these regions are reinforced with doublers or reinforcements, thereby increasing their stiffness locally. As such, the concept of tailoring fiber paths around a cut-out in a composite laminate can create the same effect without adding weight and is an interesting optimization problem. In composites design, these cut-outs and openings would introduce breaks in the continuity of fiber paths, thereby significantly increasing stress concentrations in their vicinities. A variable stiffness design could be used to improve the performance of these cut-outs.

Several research works investigated tailoring composite laminates around cut-outs using a variable stiffness approach under various loading conditions. Two studies completed by Jegley et al [14] [15] are especially interesting as the panel was tailored to reduce the stress concentration around the hole and improve the load carrying capacity [14] [15]. The results showed that the buckling load was $18 \%$ higher and the ultimate failure was $15 \%$ higher over the baseline quasi-isotropic design [14]. The variable stiffness concept was applied to an ovoid fuselage cut-out undergoing an axial compression by Wu et al [16]. The cylindrical shell with cutout maintained $91 \%$ of the axial stiffness when experimentally tested in compression and $85 \%$ of the buckling load when compared to the case with no cut-out. Large deflections were measured radially outward in the cut-out region and there were no comparisons to a quasi-Isotropic 
laminate [16]. Improving the load carrying capacity of a fuselage with cut-out was further investigated by Alhajamad et al [17]. The buckling and first ply failure due to shear were explored on a fuselage cut-out undergoing internal pressure and shear loading. The results showed that the VS design did not improve the load carrying capacity compared to a quasi-Isotropic laminate [17].

One reason could be that the fibers break at the cut-outs and are not continuous around the holes leading to stress concentrations and reduced stiffness. A technique to prevent the fibers breaking at the cut-out was first demonstrated by Yau and Chou, who created continuous fibers around holes by inserting metal pins into woven fabric prior to curing [18]. The resulting laminate was proven to have a marked improvement of $8 \%$ in tension over the one with drilled hole. A similar technique was utilized in a study conducted by Durante and Langella [19], where an improvement of $15 \%$ was found in bolted tension, when the fibers curved around the pin. Although there was an improvement, the manufacturing method was complex and there were significant variations in test results [19]. A major issue found with inserted pin technique is the resulting large gap around the hole that leads to complex laminate behaviour that cannot be accurately predicted. Therefore, preliminary design approaches were conducted by Hyer and Lee [20], in which they used a gradient search and sensitivity analysis techniques with a Finite Element Method (FEM) to improve buckling performance of a centrally located circular cut-out undergoing compression loading but the resulting design was not manufacturable. Huang and Haftka [21] repeated the study with a tension boundary condition and a similar optimization approach. The results showed that the fiber angles follow a concentric path around the hole, while tending to the far field fiber directions away from it [21]. Tailoring of the fiber paths around a hole was also examined by Lopes et al in a study to increase the buckling and first ply failure of composite plate with a centrally located circular cut-out; however, they considered discontinuous variable stiffness paths [22]. The results also indicated that the optimally placed tows would tend to steer around the cut-out. Montemurruo and Catapano used a B-Spline and genetic algorithm approach to optimize a panel with a centrally located circular cut-out undergoing a biaxial tension loading [23]. The results of the most optimal ply showed that the fiber directions tended to curve around the cut-out as well. Furthermore, a study on bolted 
composites by Gustafson using FEM showed aligning fibers along with the principal stress directions can improve the failure load by $36 \%$ to $64 \%$ over a quasi-Isotropic laminate. The resulting streamline plot of the fiber paths showed that the fiber paths tended to continuously curve around the bolted regions [24].

Many of the fiber paths that were found from the techniques described in the previous studies were not manufacturable due to restrictions in the steering radius in conventional AFP machines. The limitations arise from the material itself as the flat rectangular tow is unable to steer in large radii without the tow wrinkling or lifting up. AFP machines are also limited to placing tow against a planar surface and therefore freeform support less placement of fibers is not possible.
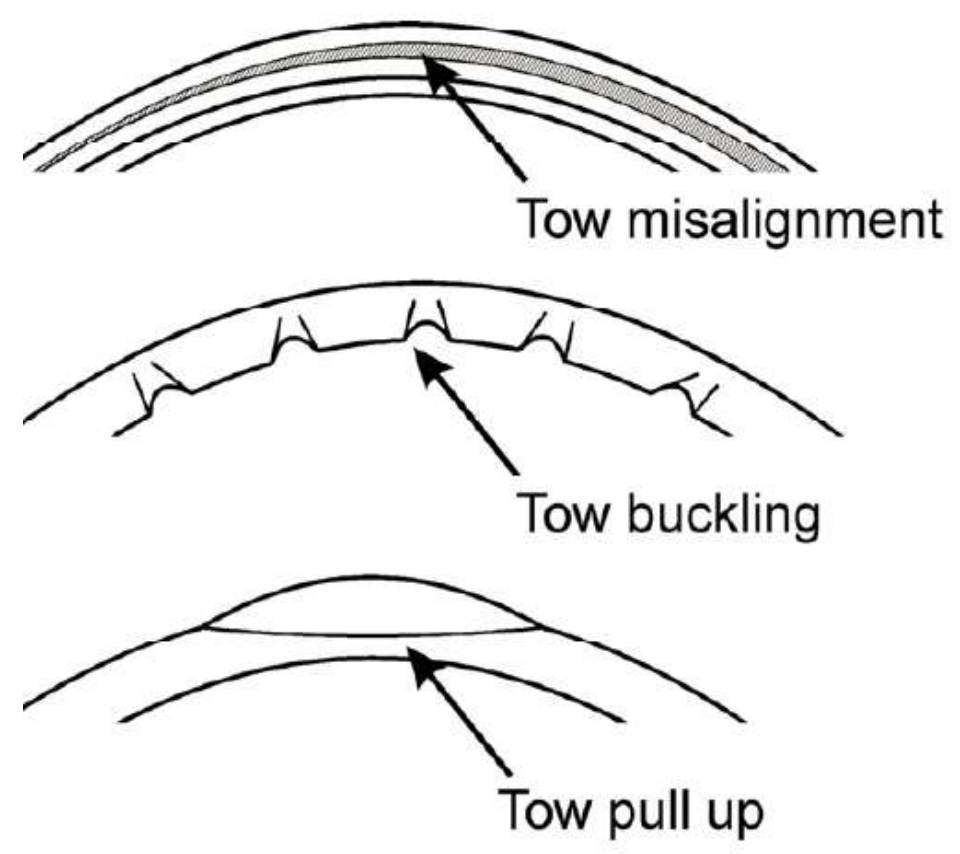

Figure 2.5 Examples of defects caused by tow steering [2] 


\subsection{D printing of Composites}

AFP technology is currently limited by size as smaller components are difficult to manufacture due to tow dimensions or the reachability of the machine. Therefore, small to medium sized components such as compressor blades are relatively difficult for machines to lay tape onto, and continue to be laminated by technicians.

A solution to the part size and steering radius issue is potentially Additive Manufacturing. Additive Manufacturing has introduced revolutionary methods towards manufacturing highly optimised geometry or near net parts. Conventional methodology includes Fused Filament Fabrication, Direct Metal Laser Sintering, or Selective Laser Annealing. Many of the methodologies are chiefly used for manufacturing thermoplastic components on the small scale, although there are methodologies in place for the manufacturing of metallic components. On the composite side, there have been materials that are infused by discontinuous synthetic fibers or particulates that increase the performance of the final part in comparison to the unreinforced matrix. While the use of additive manufacturing of composite materials has mainly been limited to discontinuous fibers composites, innovations by Markforged have enabled the deposit of continuous carbon fibers in a Nylon matrix in between layers of thermoplastic [ref?]. The use of continuous fibers enhances the performance of the final part by a factor of 10 according to Markforged, but the closed source nature and limited Computer Aided Manufacturing (CAM) ability of their Eiger software results in immense restrictions in what one is able to program in terms of the fiber directions in each layer. Dickson et al [25] were able to use the continuous carbon fiber filament from Markforged on a modified Prusa i3 for their own custom laminates. Further work by Blok et al [26] demonstrated the extreme steering angle when 3d printing continuous carbon fiber. The results of their work can be seen in Figure 2.6. 


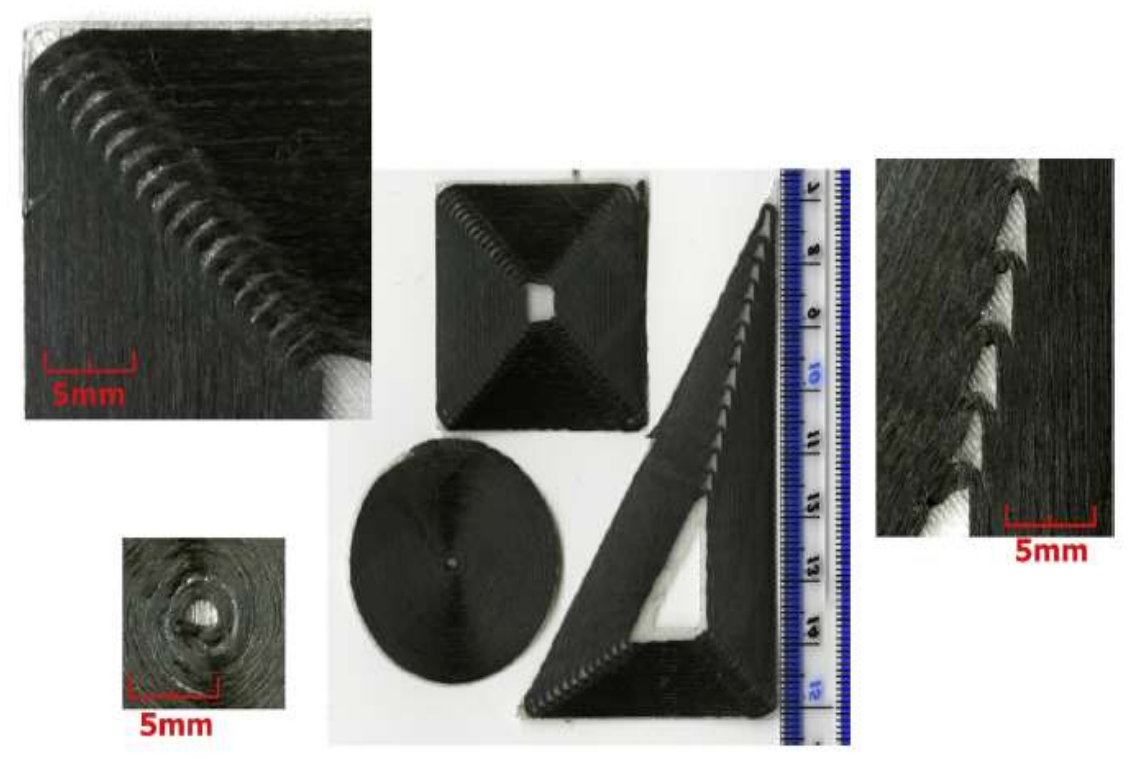

Figure 2.6 Steering capability demonstrated by 3d printing of continuous carbon fibers [26]

Several other continuous carbon filament methodologies have been implemented or proposed. Pruss \& Vietor [27] and Tian et al. [28] independently developed a FFF head that combined the dry fiber with the molten Polylactic Acid (PLA). The separate feed system introduces complexity to the manufacturing process and makes it difficult to cut the fiber in between passes. To solve the issue of impregnating the filament, $\mathrm{Hu}$ et al [29]developed a prepreg material from (PLA) and a carbon filament. The final products for all approaches had poor fiber ratios as well as poor impregnation. Alternatively, Invernizzi et al. [30] used a UV curing system for additively manufacturing carbon fiber and fiberglass, but the mechanical properties of the final part were poor.

Multiaxis CFF has been explored by Backer et al. [31] who attached a CFF head to a Kuka Kr10 robot and successfully manufactured continuous carbon fiber parts in 5 axis. Some commercial endeavours have investigated multiaxis CFF such as Arevo and Piulabs, which similarly use a CFF head attached to an industrial robot. Continuous carbon fibers can also be used to manufacture parts that are out of plane or 3d lattices as seen in Figure 2.7 [32]. 


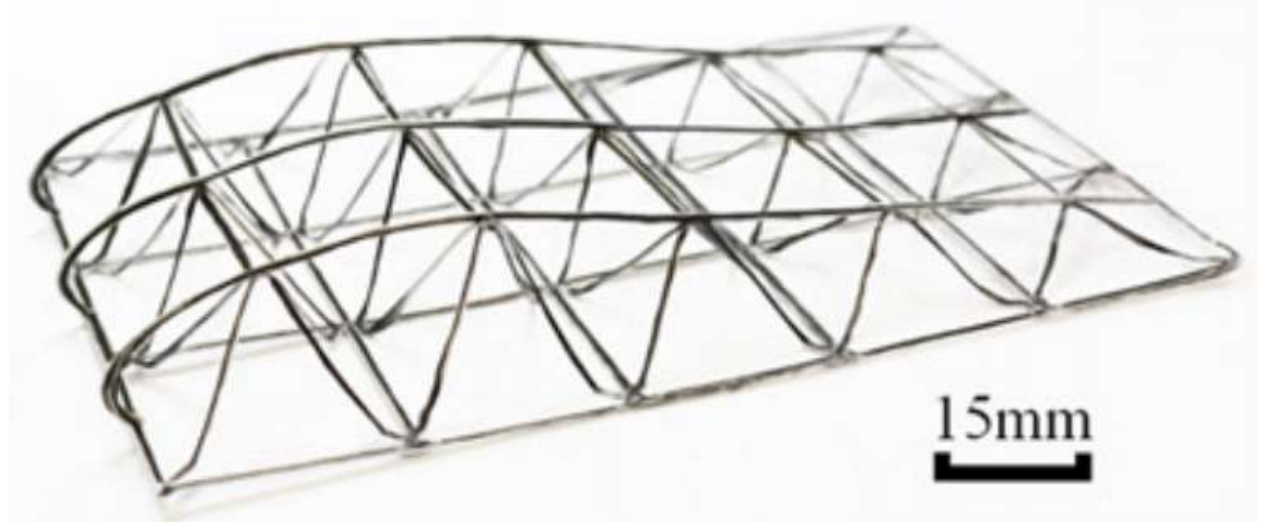

Figure 2.7 Out of plane 3D printing of continuous carbon fibers [32]

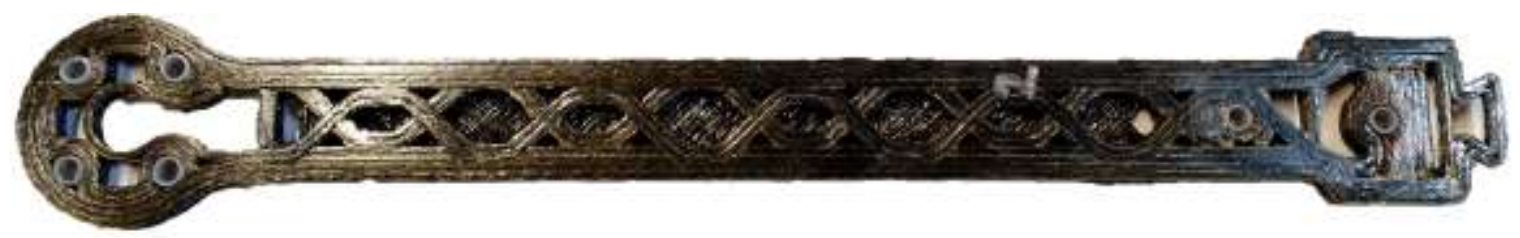

Figure 2.8 Drone arm printed using continuous carbon fiber [31]

\subsection{Inspections}

Additive Manufacturing is a key part of the Industry 4.0 revolution that allows for highly complex designs and a decentralised manufacturing network [33]. Parts have been difficult to manufacture using traditional techniques are more feasible with additive techniques [34] [35]. One such technique of interest is Fused Filament Fabrication (FFF), which can be used to produce thermoplastic parts without the need for an injection moulding system. Thermoplastics are increasingly becoming of interest in the Aerospace and Automotive industry due to materials such as PEEK (Polyether Ether Ketone) offering high performance at elevated temperatures [36]. Currently FFF has seen limited use in production due to a conservative mindset and the relative maturity of the process. For greater adoption by industry outside of the traditional use as a rapid prototyping tool, there are several issues that need to be addressed for full production to be feasible. The most pressing concern with respect to the Aerospace market would be quality. FFF 
can potentially replace many components in aircraft but the process is known to produce defects that create inconsistent quality and may not meet the standards of the Aerospace market [37].

As quality is a major concern for FFF manufacturing, many researchers have been investigating the process and quantifying how various parameters may affect the strength of the final part. FFF manufacturing consists of parameters and procedures that greatly vary from traditional approaches. There are a plethora of settings and configurations such the raster angles, part orientation during print, and infill patterns that can change the way a part can perform compared to one that is traditionally manufactured [38] [39]. Due to the numerous parameters, the manufacturing process itself often is the cause of defects, and they often appear due to part orientation causing overhangs [40]. Defects caused by overhangs can often be avoided by reorienting the part in a favourable orientation, but raster angle and the layer height can have an impact on the quality of the part even when favorably oriented [40]. On the impact of layer thickness, Rankouhi et al [41] examined the effects of layer thickness on the performance of the part. They found that the layer thickness has an impact on the strength of the part and that in cross ply areas, air gaps are formed which contribute to a reduction in the strength of the part. Torrado et al [39] also found similar air gaps when inspecting the cross section of failed coupons. Furthermore, a study by Fayazbaksh et al [42] investigated the impacts of defects on FFF manufactured ASTM D638 coupons of various stacking sequences. In their study, defects were intentionally introduced as missing extrudate in each layer, thereby forming a gap in that layer. The results confirmed that parts manufactured by FFF have anisotropic properties as a coupon with a $[90]_{24}$ stacking sequence had a mean strength that was $46.7 \%$ less than the $[0]_{24}$ stackup. Furthermore, a $20.5 \%$ reduction in the tensile strength was observed with the various defects, indicating that potential manufacturing defects can have a major impact on performance of the part. A noteworthy finding was that the $[90]_{24}$ with no defects had a high coefficient of variation of $12.6 \%$ compared to the other samples. Such a high variation can indicate that the FFF process may be inducing a defect when extruding material in this direction. Similarly, Koch et al [43] investigated the effects of raster angle and the observed anisotropic effects of FFF manufactured components compared to injection moulded ASTM D638 coupons. They observed that the coupon with only 0 degree layers had $98 \%$ of the performance of the injection moulded coupon. 
They also observed that the 90 degree raster angles would result in a reduction of the strength of $69.86 \%$ relative to the injection moulded coupon. Similarly, to Fayazbaksh et al [42], a high variation in the 90 degree coupons was observed. They attributed it to the geometry of each extrudate being a circular bead that would leave a gap when in contact with the adjacent extrudate.

FFF defects have proven to be a significant concern when the part may be used in primary structural applications. No manufacturing process is free from defects; therefore, it is best to mitigate the potential for unsatisfactory parts to exit production. For conventionally manufactured components, the inspection is done post-process through various means such as Coordinate measuring (CM) and 3D scanning for external features; Ultrasound and X-ray for internal features. Many of these techniques have been applied towards FFF manufactured parts [44]. 3D scanning was used by Pathak et al [45] as an alternative to CM for Geometric Dimension and Tolerancing (GD\&T) confirmation. Ultrasound was used by Na et al [46] to inspect a FFF part. They were able to image the defects and capture the geometry of internal features such as infill and delamination up to a resolution of $50 \mu \mathrm{m}$.

FFF is a time dependant process, where the greatest incurred cost is usually the operation of the machine. Therefore, it would not make sense to complete a part that would be considered defective. Goh et al [47] proposed an integrated approach to FFF manufacturing where an in-situ inspection technique would be used to enhance the post process inspection techniques to further ensure the quality and reliability of the produced part. This way a complete description of the parts performance can be estimated and compared against acceptable metrics, while at the same time saving manufacturing time in the event a defect is detected during the manufacturing process.

In process or in-situ inspection for FFF has been investigated by various means, such as thermography or acoustic emission, but the most prominent was through optical inspection using a digital imaging system [48]. A digital imaging system was used by Straub [49] [50] [51] to capture whether the part was beginning to warp based on an image processing algorithm that looked for dark areas in the image, which in this application would be the gap between the build 
plate and the part. The system is capable of detecting the warping, but the infill or the geometry of the part was not measured. An augmented realty approach was undertaken by Ceruti et al [52], who used a digital imaging system to supervise a print while superimposing the expected 3D model on top of the printed part. The resulting system was able to discern differences that are entirely qualitative, while internal features such as infill are not measurable. Wu et al [53] applied machine learning and image classification to identify defects during the printing process. The methodology used a digital camera to image each layer, which is then run through an image classification algorithm to look for pre-defined defects. The system is capable of finding the defects in the infill of a part, but there were no quantifiable results such as the percentage deviation of the part from the baseline.

An alternative approach was investigated by Cummings et al [54] in which they used ultrasonic sensors adhered to the bed of the printer to inspect the part as it was being printed. The system consisted of one transmitter for the ultrasound and three receivers that were situated around that part. The response of the system was compared to a Finite Element modal analysis of the part on the bed. The system is able to detect delamination in the part but is unable to quantify the defect and lacks resolution to capture the gaps in the extrudate.

The methods previously mentioned fail to quantifiably determine the voids and defects that were investigated by Fayazbaksh et al [42] and Koch et al [43]. An unexpected industry to take inspiration from would be electronics industry and their need for automated dispensation of solder. A Laser line scanner was developed in the late 90s for inspection of solder paste screening in Surface Mount Technology (SMT) bonding on Printed Circuit Boards (PCB) [55]. The paste is deposited similarly to FFF manufacturing, where a gantry system deposits the solder material on the board on the etched areas. As the surface area for the contact region for SMT's are in the micron range, it is necessary to ensure that all contact regions have the solder placed properly and are free of defects. In doing so it would be possible to determine if a region had too little or too much of the solder paste. The methodology used is known as Optical triangulation, where the object is illuminated by a narrow laser beam. The reflections scattered from the object can be read by a sensor to triangulate height values across the length of the laser beam. This method allows for fast scans without slowing down production. 
The laser scanning machine described previously [55] would not be feasible for integration in a commercial FFF 3D printer as the size was that of a refrigerator. Fortunately, triangulation technology has advanced enough in the many decades since publication by Horijon et al [55] and scanners are now available commercially at the size of a deck of cards with greater sensitivity.

Precise measurement of each layer can be used to build an accurate representation of the part. The means of correlating the effects of gaps and overlaps was investigated by Fayazbaksh et al [56], where the image correlation library in MATLAB was used to map the gaps and overlaps to a Finite Element Model using a technique known as Defect Layer Method (DLM). The DLM was able to accurately estimate the panel's performance, but limitations are in the method of imaging through thermography or predictions through the CAD model. If the panel is imaged through laser triangulation, a more detailed and accurate model can be generated. 


\section{CHAPTER 3 CURVILINEAR CONTINUOUS VARIABLE STIFFNESS FOR IMPROVED OPEN HOLE TENSION}

Open hole tension is an important area for design optimisation and stress reduction as many aerospace structures feature large discontinuities in their design. In the fuselage of an aircraft this is usually in the form of cut-outs for windows, whereas in other applications it can be cut-outs for access panels and similar design features.

Reducing stress concentrations around cur-outs has been a topic of investigation by many researchers as presented in the literature review. Many of these works result in fiber paths that are not feasible, or impractical to produce. Therefore, a novel strategy is proposed which utilises the analogy of fluid flow around a cylinder, which is commonly used to describe stress lines around cut-outs in many stress analysis textbooks [57]. Such an analogy can be helpful as fluids tend to flow over a surface such that where stress cannot exist, the fluid will not exist, which is in this case the space inside the hole. To improve stress concentrations, such a property of fluid flow would be useful in curving the fiber path around the hole with slow transitions and continuous fibers.

To validate this concept, ASTM D5766 coupons were manufactured using an FFF process. Although it would be simpler to use FEA to model the trajectory, such a result is meaningless if the design is ultimately not manufacturable. According to the literature that was presented, FFF does exhibit orthotropic properties, and therefore can serve as a good surrogate for future designs that utilise a continuous fiber. The specimen geometry, stacking sequence, and testing procedure are designed per ASTM D5766. The extrudate paths were generated using the solution to a rotating cylinder in a cross flow and printed on a commercial FFF 3D printed. 


\subsection{Specimens geometry and stacking sequence}

A specimen with a centrally located hole according to the configuration B per ASTM D5766 is selected in this research for open-hole tensile strength testing. The width, length, and thickness of the specimen are $36 \mathrm{~mm}, 150 \mathrm{~mm}$, and $2.24 \mathrm{~mm}$, respectively with a specimen grip length of $35 \mathrm{~mm}$. The notch consists of a hole centrally located by length and width of the specimen. Furthermore, experimental testing is extended to investigate the influence of the specimen width to hole diameter (w/D). Therefore, two cases with w/D of 2 and 4 are designed and manufactured. The standard laminate is balanced and symmetric, and has multi-directional fiber orientations. To demonstrate improvements in load carrying capacity that can be achieved using curvilinear variable stiffness design, an optimum constant stiffness design is considered as the baseline with filaments aligned along with the loading direction $\left(0^{\circ}\right)$. Previous study [17] suggested including $\pm 45^{\circ}$ layers can improve design robustness under off-design conditions. As a result, a $\left[ \pm 45 / 0_{6}\right]_{s}$ stacking sequence is considered as the baseline, which has 16 layers, each 0.14 $\mathrm{mm}$ in thickness. For curvilinear variable stiffness design, $0^{\circ}$ layers are replaced with optimum load paths resulting in a $\left[ \pm 45 /(\mathrm{VS})_{6}\right]_{s}$ stacking sequence, where VS denotes curvilinear Variable Stiffness (VS) layers. According to ASTM D5766, five specimens per test conditions are designed, manufactured, and tested,

Hole diameter (D) and specimen thickness are measured using a dial caliper and reported to the nearest $0.025 \mathrm{~mm}$ (0.001 in). A micrometer is used to measure the specimen width, which is also reported to the nearest $0.025 \mathrm{~mm}(0.001 \mathrm{in})$. Since strain response local to the hole is of interest, one extensometer to the specimen edge is attached and it is ensured that the hole is located within the extensometer gage section. A testing speed of $2 \mathrm{~mm} / \mathrm{min}$ is chosen to give rupture within 0.5 to 5 -min testing time and force versus strain is recorded with a target minimum of 100 data points. 


\subsection{Coupons load paths}

An analogy to stress trajectories in the case of open hole tension can be taken from fluid flow around a cylinder. Such an analogy can be helpful, as fluids tend to flow over a surface smoothly in one continuous streamline to take the path of least resistance. To improve stress concentrations, such a property of fluid flow would be useful in curving the fiber path around the hole with slow transitions and continuous fibers [28].

To model these load paths to be used in 3D printing, the field equations for potential flow were considered as the trajectories of a distance $r$ from the reference point. The analytical solution for the potential flow around a cylinder allows determining the load paths at specific distances from center of the hole that were proportional to the width of the bead.

The velocity potential can be obtained by the analytical solution to the unit flow around a cylinder:

$$
\phi(r, \theta)=r\left(1+\frac{R^{2}}{r^{2}}\right) \cos \theta
$$

Where, $r$ is the distance from the hole center, $\theta$ is the angle from the $x$-axis, and $R$ represents the hole radius [29].

The partial derivatives of the potential function (Eq. 1) can be written as:

$$
\begin{gathered}
V_{r}=\frac{\partial \phi}{\partial r}=\left(1-\frac{R^{2}}{r^{2}}\right) \cos \theta \\
V_{\theta}=\frac{1}{r} \frac{\partial \phi}{\partial \theta}=-\left(1+\frac{R^{2}}{r^{2}}\right) \sin \theta
\end{gathered}
$$

Where $V_{r}$ is the radial component and $V_{\theta}$ denotes the tangential component in the polar reference frame.

The superposition of three potentials were taken with respect to the origin, with the spacing between them being the width of the specimen. The superposition principle allowed for the creation of a boundary at the edge of the specimen, by creating essentially what are "virtual" holes outside the coupon boundary. The corresponding mirrored vortexes would eliminate each other's influence, thereby generating only a $u$ component at the boundary. Adding the super 
position is necessary, as without the cancellation, the load paths would exit the coupon boundary.

The velocity components were then calculated across a mesh that represented the boundaries of the test specimen described in Section 2.1. The velocity components in the polar reference frame were then translated to the Cartesian reference frame to calculate the trajectories. Figure 3.1 shows the trajectories with the load paths overlaid to illustrate how the velocity components calculated at each point in the mesh are interpolated into the load paths.

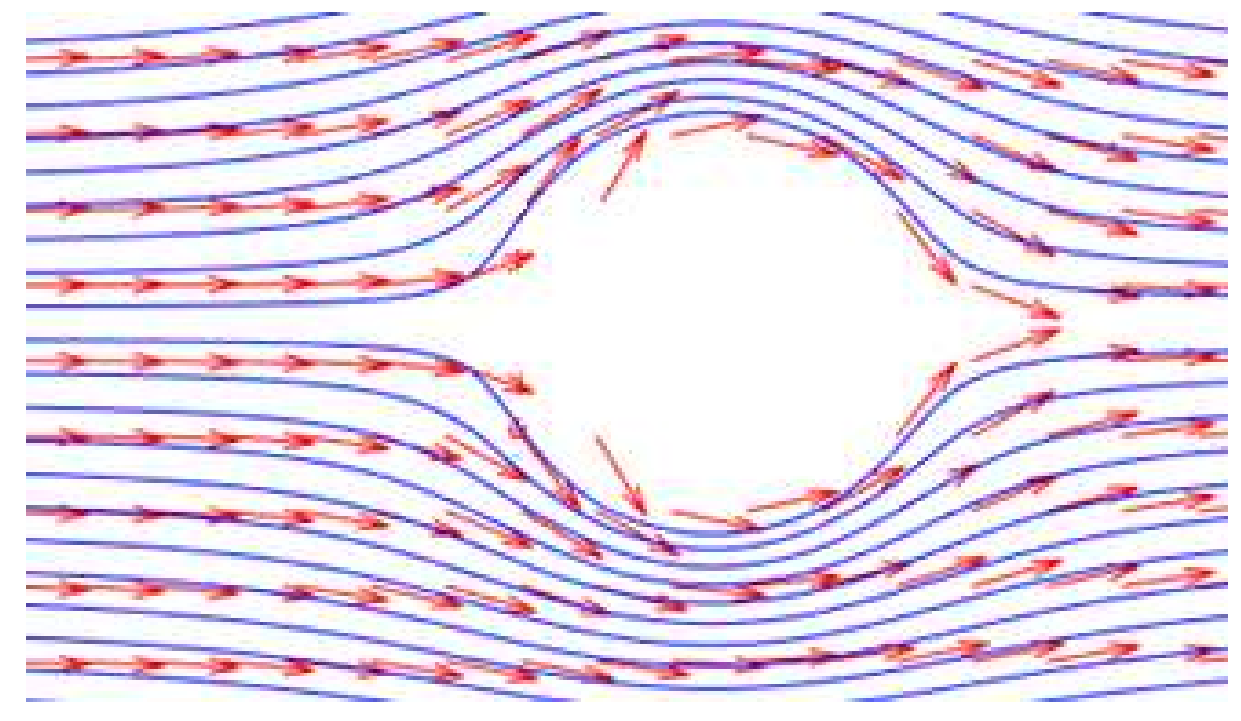

Figure 3.1. Trajectories with load paths overlaid.

Each streamline was considered as the centerline of a bead, with the spacing set as the bead width. Besides generating the material deposition trajectories, various coefficients are determined to account for the manufacturing parameters like the amount of filament deposited per distance traveled, bead thickness, and height. Many of these coefficients were determined with respect to the default settings of the Prusa i3 M2 printer.

As shown in Figure 1, imposing the same spacing between the load trajectories caused them to converge around the hole indicating the stress concentration induced by the hole. In manufacturing of the curvilinear variable stiffness layer, a no-overlap strategy is used to avoid a thickness buildup. As a result, the beads around the hole just touch, whereas there are gaps between the beads in regions far from the hole. For $\pm 45^{\circ}$ raster layers, there is no gap and/or 
overlap between subsequent beads and they break when reaching the hole as opposed to curvilinear paths. The generated streamlines in MATLAB for the specimen with $w / D=4$ can be seen in Figure 3.2.

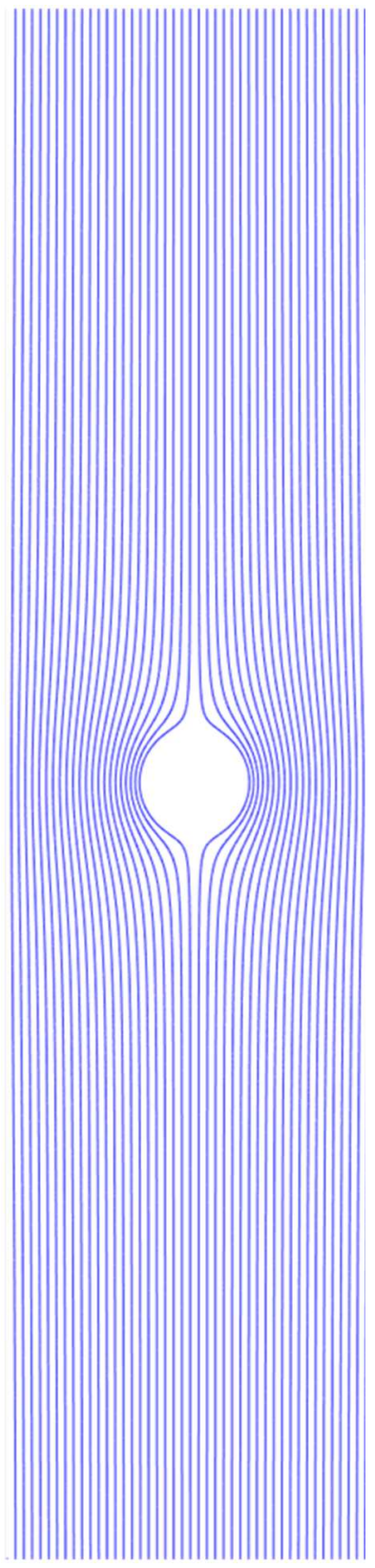

(a)

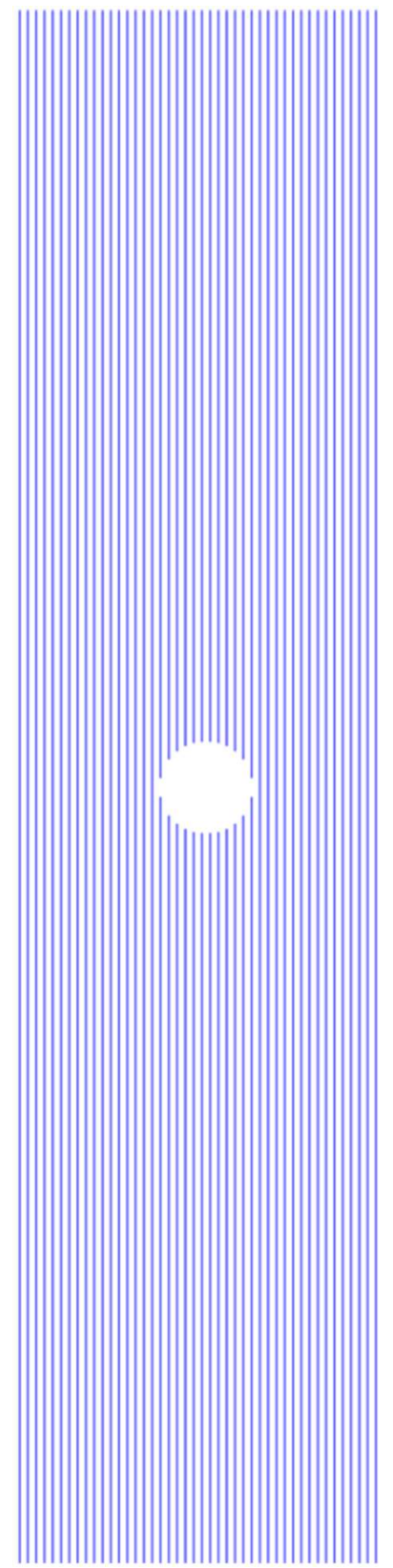

(b)

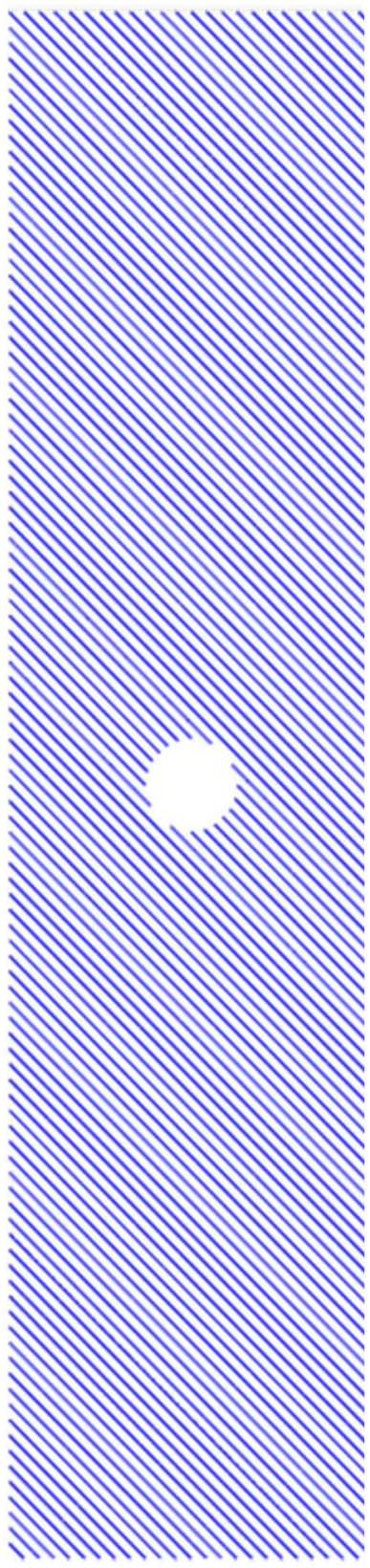

(c)

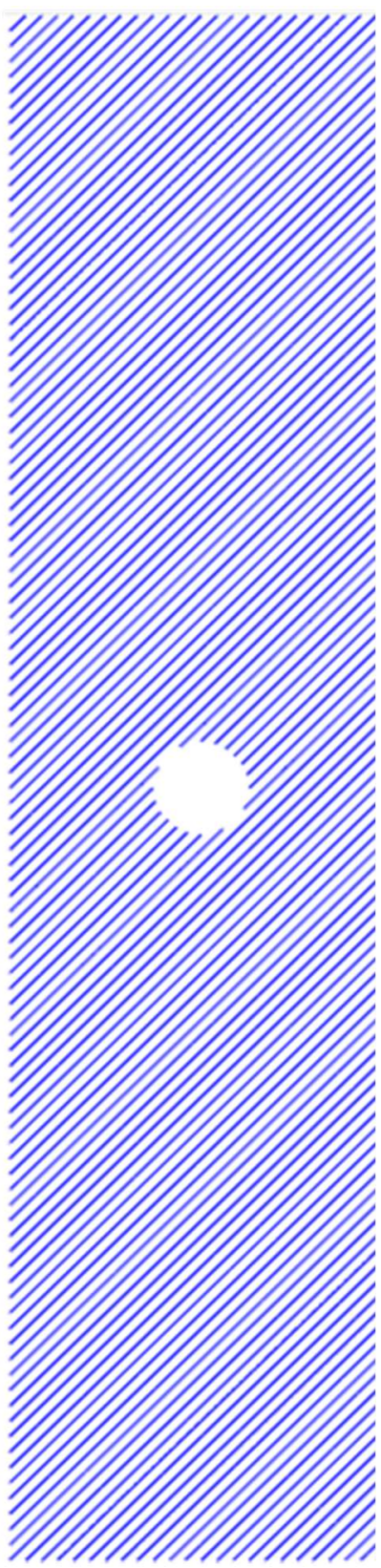

(d)

Figure 3.2 The load paths for open-hole tensile specimen with $w / D=4$ : (a) curvilinear variable stiffness, (b) $0^{\circ}$, (c) $-45^{\circ}$, and (d) $+45^{\circ}$. 


\subsection{Specimens manufacturing}

Currently, there is no commercially available tool for converting custom trajectories to GCODE to be used by a 3D printer. Therefore, a MATLAB script was developed for the purposes of turning mathematical expressions into GCODE. The process for generating GCODE for curvilinear extrudate paths is quite straightforward when the path is linearly interpolated through. By setting the step size between linear interpolations, the total curvilinear path can be interpolated with very short linear movements. Initial testing was done on a Creality CR-10 to determine the parameters needed for proper deposition of the extrudate. The major parameter was determining the step size, as having the step size can influence the amount of material deposited due to round off errors and truncation on the hardware side. In Figure 3.3, areas of over and under extrusion can be seen, which is a direct result of the linear interpolation spacing. Filament retraction was also necessary to determine, as not retracting enough at the end of path is likely to drag residual material across the part. Through empirical investigation, this can be controlled and a consistent material deposition can be attained as seen in Figure 3.4. 


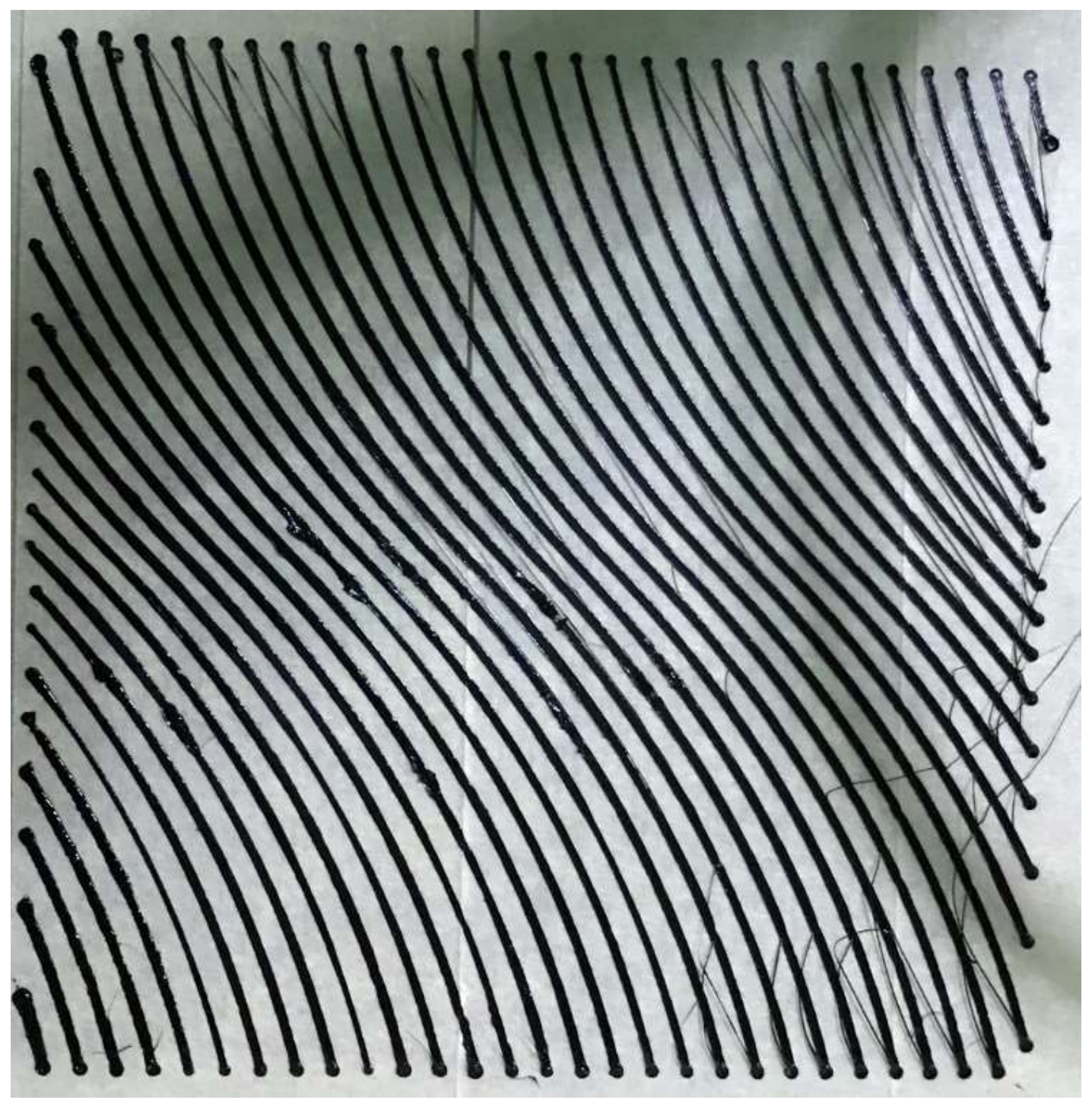

Figure 3.3 Linear Variable Stiffness test 


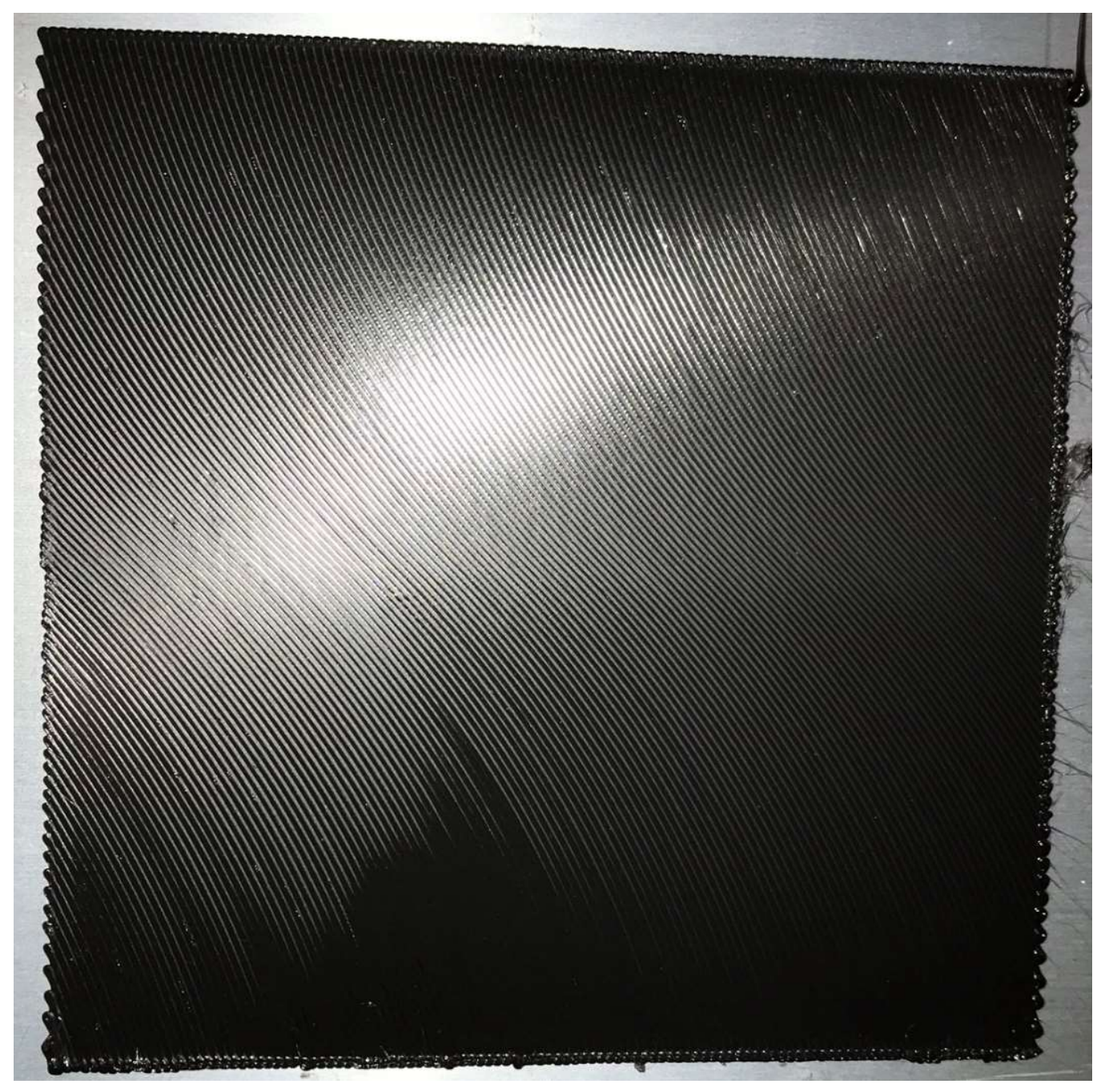

Figure 3.4 Linear Variable Stiffness print complete fill

For generation of the open hole tension coupons, the streamline solutions are used as the path for the deposition of the extrudate. For generating the $x, y$ coordinates and the amount of extrusion required, a linear spacing of points are distributed across each streamline. The points are then linearly interpolated and added to a text file as GCODE commands. Furthermore, the layer height and the start and end points are also automatically generated using the subroutine. 
Figure 0.5. Curvilinear variable stiffness path in GCODE.

\subsection{Experimental Study}

Five set of specimens are manufactured using Prusa i3 MK2S for each configuration described in Section 2.1. The manufacturing process and design parameters are as follows: $\mathrm{XYZ}$ print direction, PLA material, layer height of $0.14 \mathrm{~mm}$, nozzle and bed temperature of $215^{\circ}$ and $60^{\circ}$, respectively, and no fan cooling. Figure 3.6 shows placing of the beads along curvilinear paths during the manufacturing process. and the completed layer is shown in Figure 3.7.

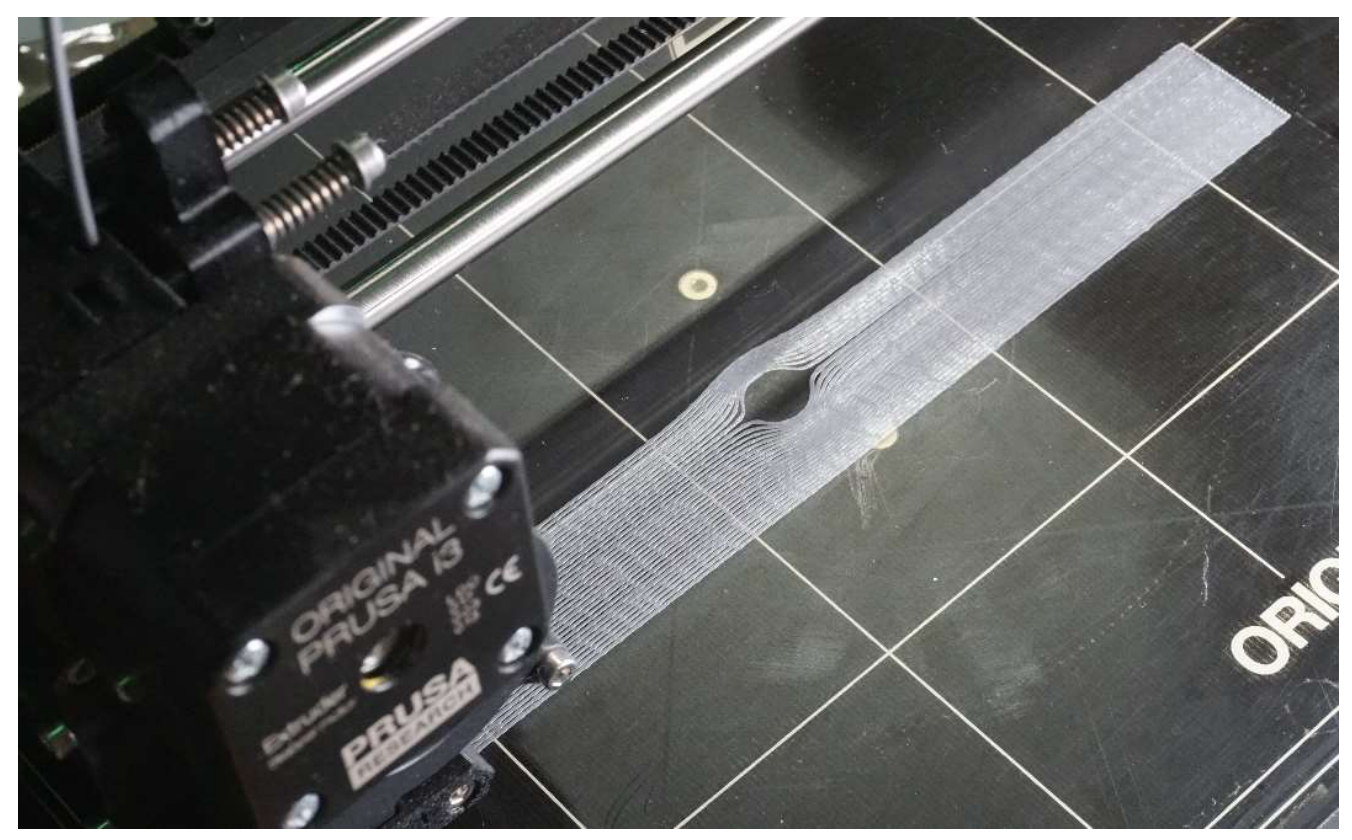

Figure 3.6. Curvilinear variable Stiffness layer during printing 


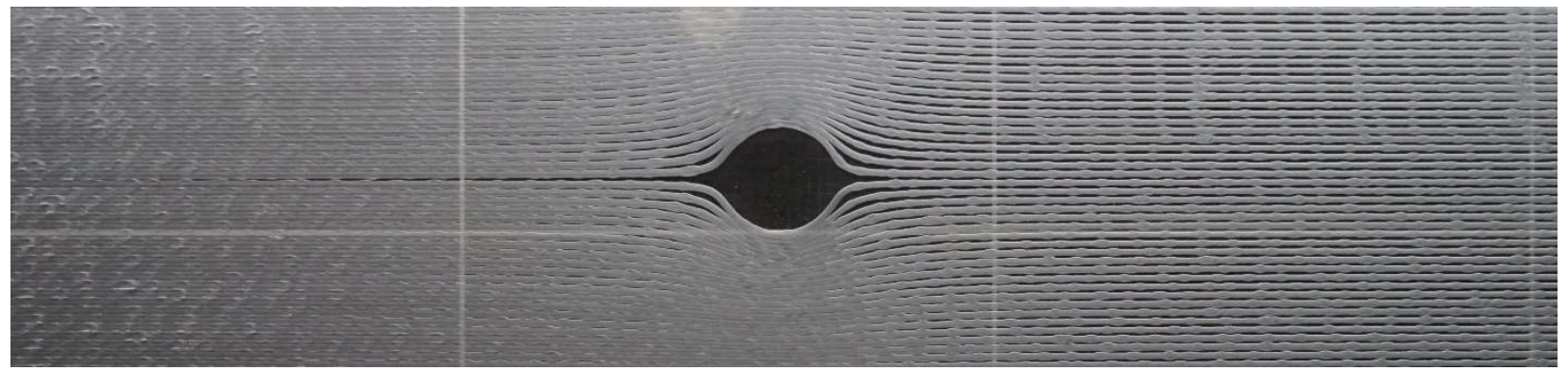

Figure 3.7. Completed curvilinear variable stiffness layer for $w / D=4$.

In addition, five sets of specimens with only straight rasters are manufactured using the same manufacturing process and design parameters explained above. Figure 0.8 shows a Variable Stiffness (VS) and Constant Stiffness (CS) specimen after 3D printing.

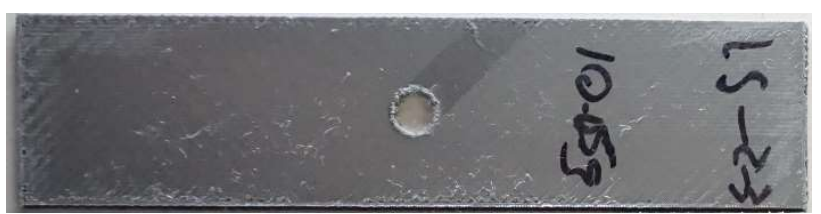

(a)

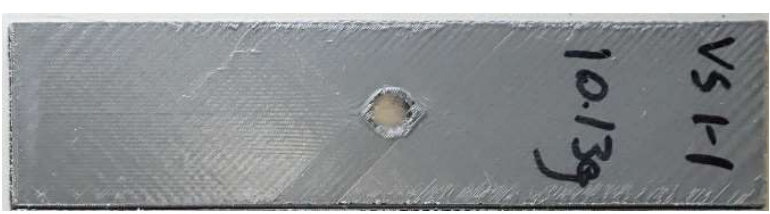

(b)

Figure 0.8. Open-hole tensile specimens with $w / D=4$ : (a) CS specimen and (b) VS specimen.

The completed specimens were close in weight variation with the Variable Stiffness (VS) specimens weighing slightly less than the Constant Stiffness (CS) specimens do. The same process is followed and five sets of VS and CS specimens are manufactured for the other configuration, $w / D=2$. Table 0.1 shows the average weight and standard deviation for the manufactured specimens.

Table 0.1. Weight variation of manufactured VS and CS specimens

\begin{tabular}{|c|c|c|l|l|}
\hline \multirow{2}{*}{ W/D ratio } & Design & Stacking sequence & $\begin{array}{c}\text { Average Weight } \\
(\mathrm{g})\end{array}$ & Standard Deviation (g) \\
\hline \multirow{2}{*}{2} & CS & {$\left[ \pm 45 / 0_{6}\right]_{s}$} & 9.57 & 0.11 \\
\cline { 2 - 5 } & VS & {$\left[ \pm 45 /(\mathrm{VS})_{6}\right]_{s}$} & 9.24 & 0.14 \\
\hline \multirow{2}{*}{4} & CS & {$\left[ \pm 45 / 0_{6}\right]_{s}$} & 10.06 & 0.03 \\
\cline { 2 - 5 } & VS & {$\left[ \pm 45 /(\mathrm{VS})_{6}\right]_{s}$} & 10.14 & 0.05 \\
\hline
\end{tabular}




\subsection{Grip Design}

All manufactured specimens are tested using a United testing machine with a $10 \mathrm{kN}$ load cell and the results are presented in Section 3. The existing grips on the machine were insufficient for the sample size due to the height and width of the test coupons. Therefore, custom grips were designed for the purposes of gripping the coupon. The grips were affixed directly to the testing head using adapters provided by United for the purposes of affixing custom grips. Additionally, an alignment jig was designed to prevent misalignment of the grips. In Figure 3.9, a CAD model of the grips is represented with $(A)$ demonstrating the use of the alignment jig.

The grips were manufactured from 4140 steel that was hardened to HRC45.Figure 3.10 shows the test set-up including a variable stiffness specimen for $w / D=4$ inside the fixture with the extensometer. 


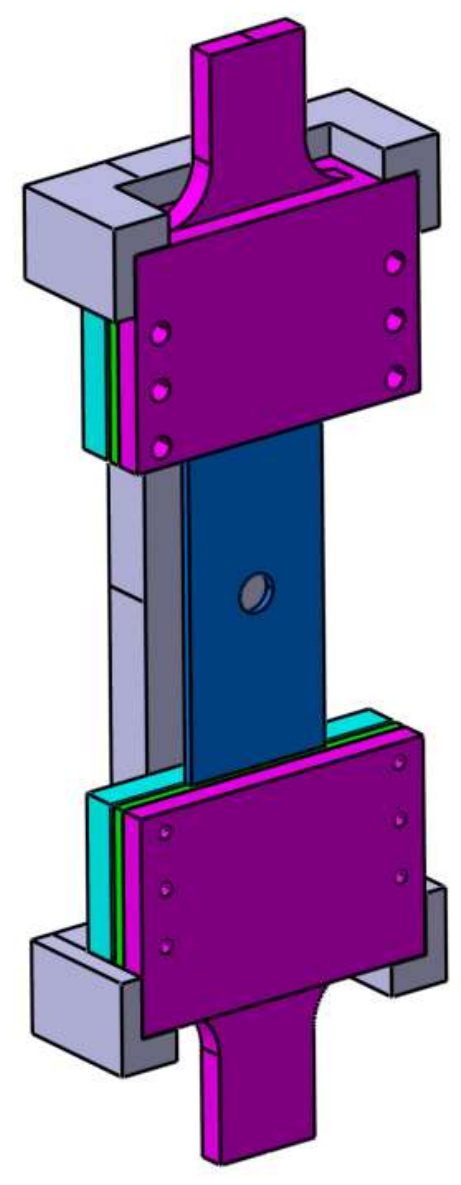

(A)

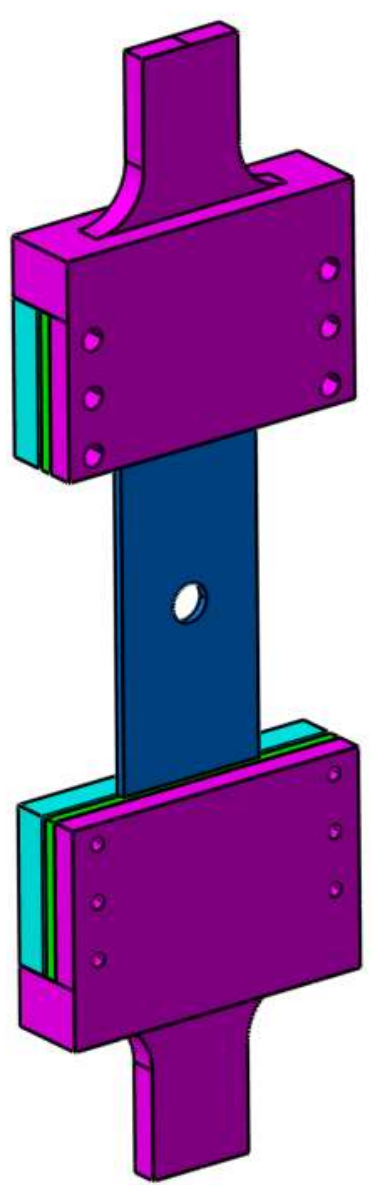

(B)

Figure 3.9 (A) Grips with Alignment Jig (B)grips with no jig 


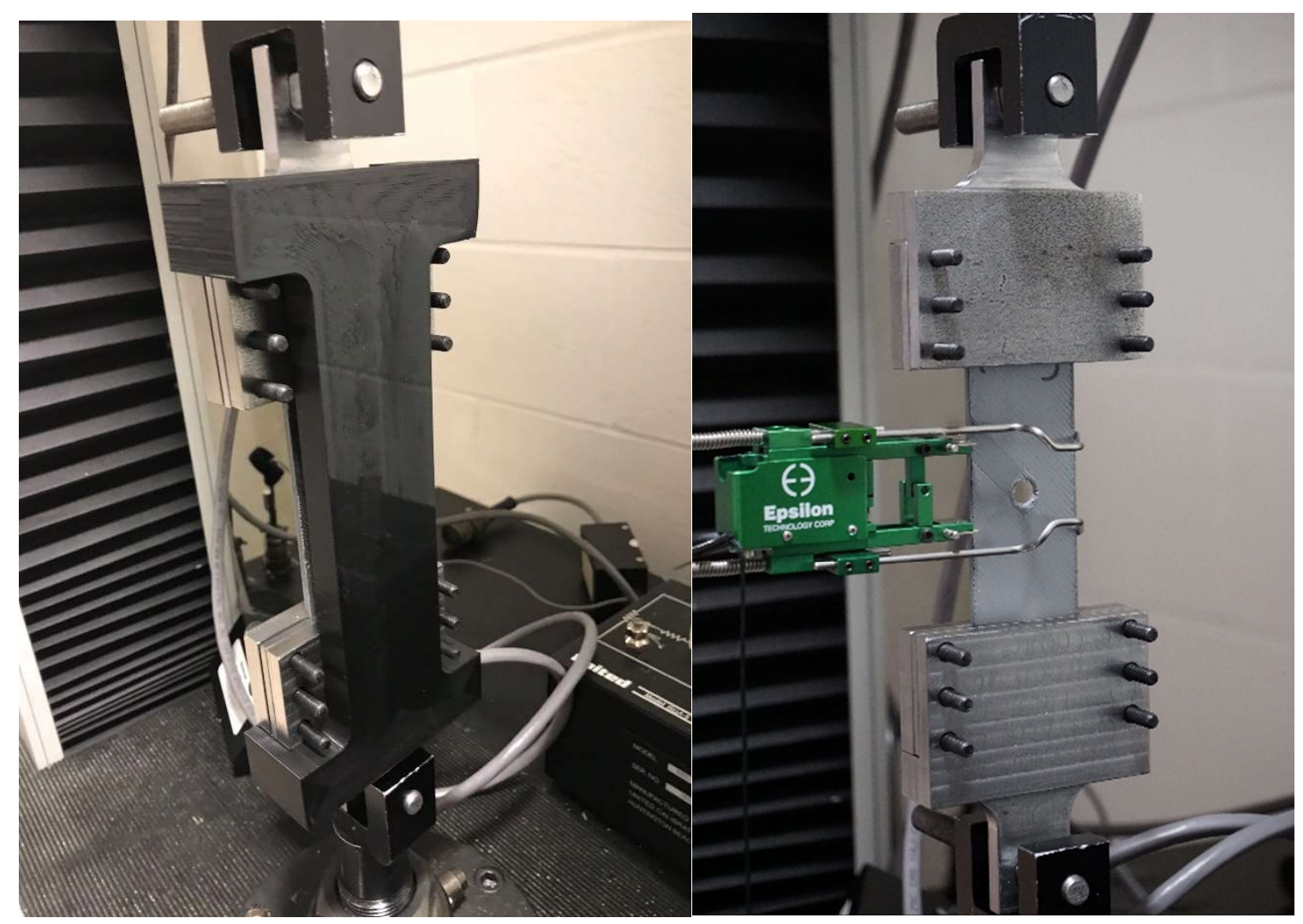

Figure 3.10. The test set-up including a variable stiffness specimen ( $w / D=4)$, the fixture, and the extensometer.

\subsection{Experimental Results}

A constant crosshead speed of $2 \mathrm{~mm} / \mathrm{min}$ is applied to the specimens until failure, while force versus strain is recorded. According to ASTM D5766, failures that occur at the hole are acceptable failure modes and are limited to Gage Middle (*GM), where they can be either Lateral $(L)$, Angled (A), or Multi-mode (M), resulting in failure mode codes of LGM, AGM, and MGM. Ultimate open-hole tensile strength is calculated based on the gross cross-sectional area, ignoring the presence of the hole. All specimens for the two configurations ( $w / D=2$ and 4 ) showed acceptable failure modes per the standard ranging from LGM and AGM to MGM. Figure 3.11 shows a variable stiffness specimen for $w / D=4$, where failure passes through the hole in the test specimen (LGM mode). 


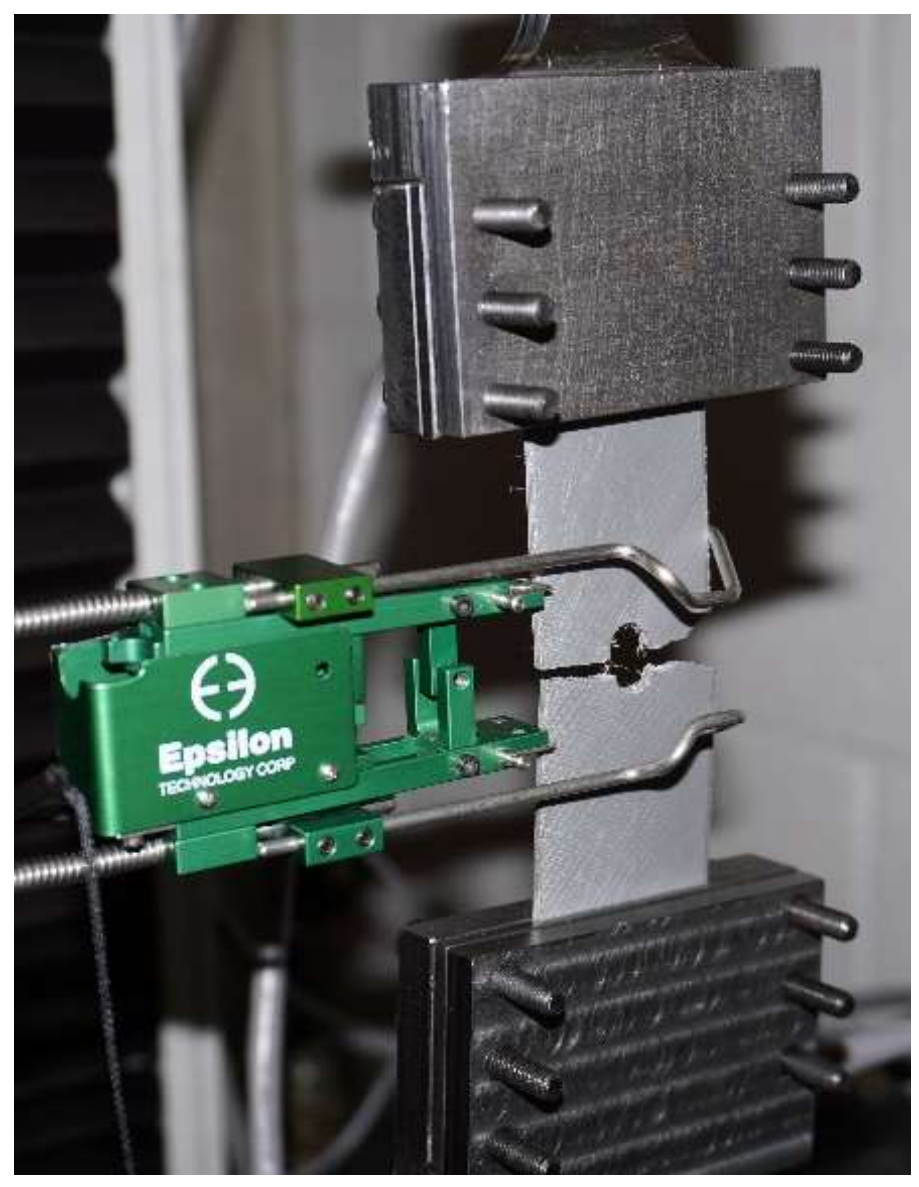

Figure 3.11. A variable stiffness specimen ( $w / D=4)$ after failure (LGM mode).

The stress-strain graph for a Constant Stiffness (CS) and a Variable Stiffness (VS) specimen with $w / D=2$ can be seen in Figure 3.12. It is evident that the use of curvilinear variable stiffness design significantly improves the failure strength for $w / D=2$ configuration, while the improvement in failure strain is limited. 


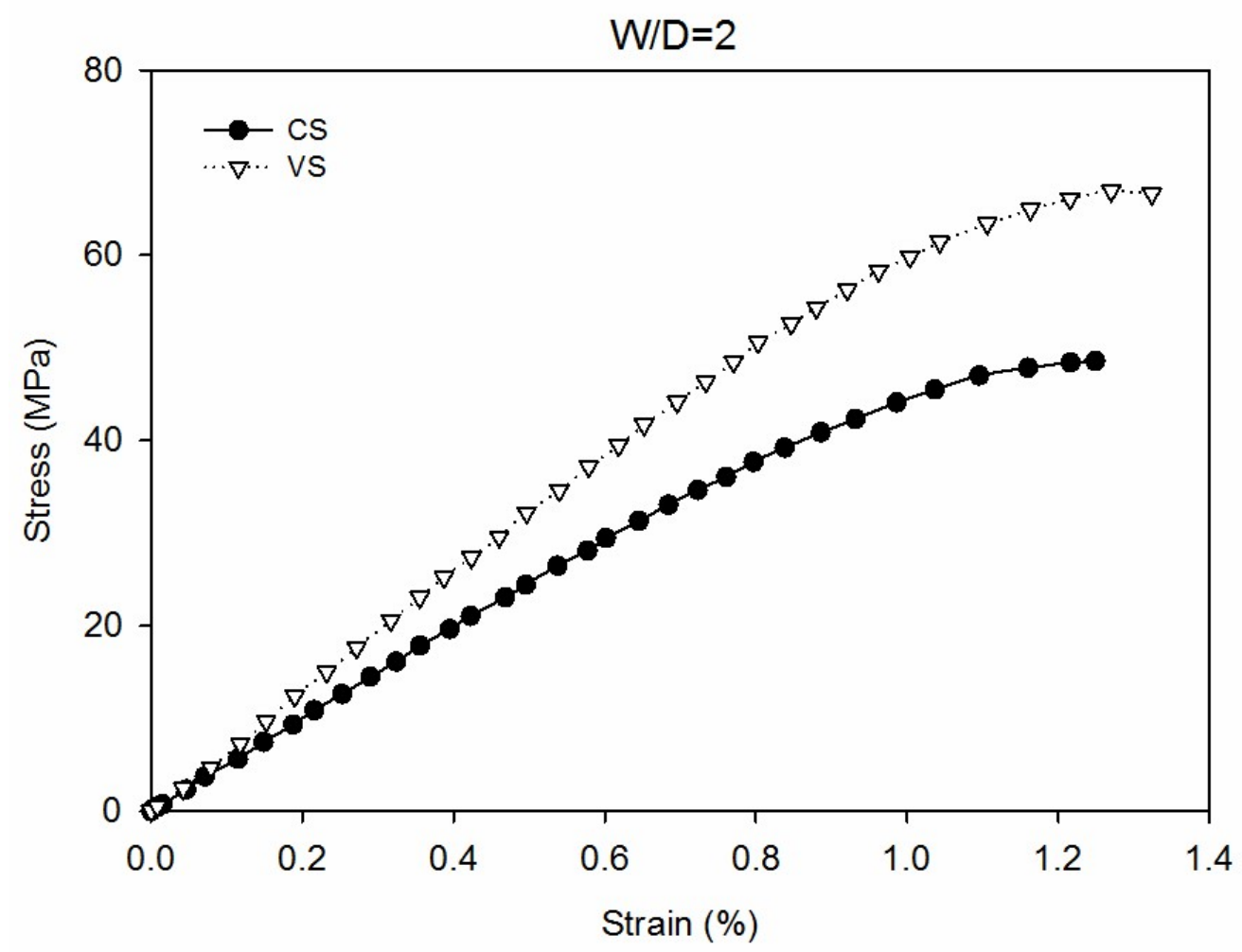

Figure 3.12. Stress-strain graph for CS and VS specimens with $w / D=2$.

It should be noted that beads are cut at the hole for the Constant Stiffness (CS) design, keeping only limited number of them around the hole. On the other hand, for the Variable Stiffness (VS) design, no bead is cut at the hole and they are all steered around the hole, filling the area around it. The difference in the materials distribution around the hole in the two cases can be clearly seen in the failed cross section imaging for CS and VS designs (Figure 3.13).

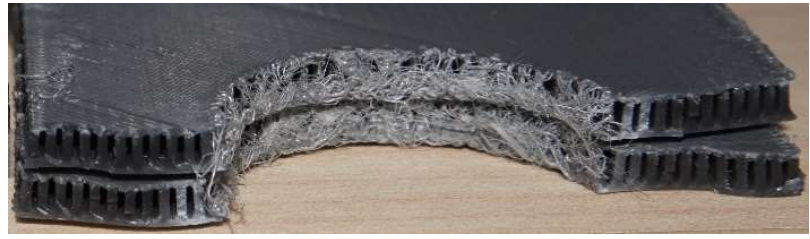

(a)

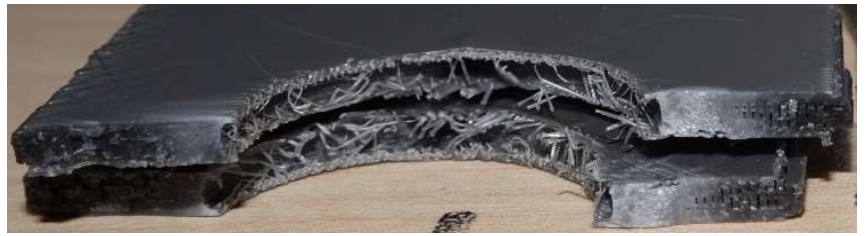

(b)

Figure 3.13. The cross sections of failed specimens (w/D=2): (a) CS design; (b) VS design.

The stress-strain graph for a Constant Stiffness (CS) and a Variable Stiffness (VS) specimen with $w / D=4$ can be seen in Figure 3.14. Unlike the previous case $(w / D=2)$, the improvement in 
failure strain is substantial with the use of curvilinear variable stiffness design, while improvement in failure stress is less pronounced.

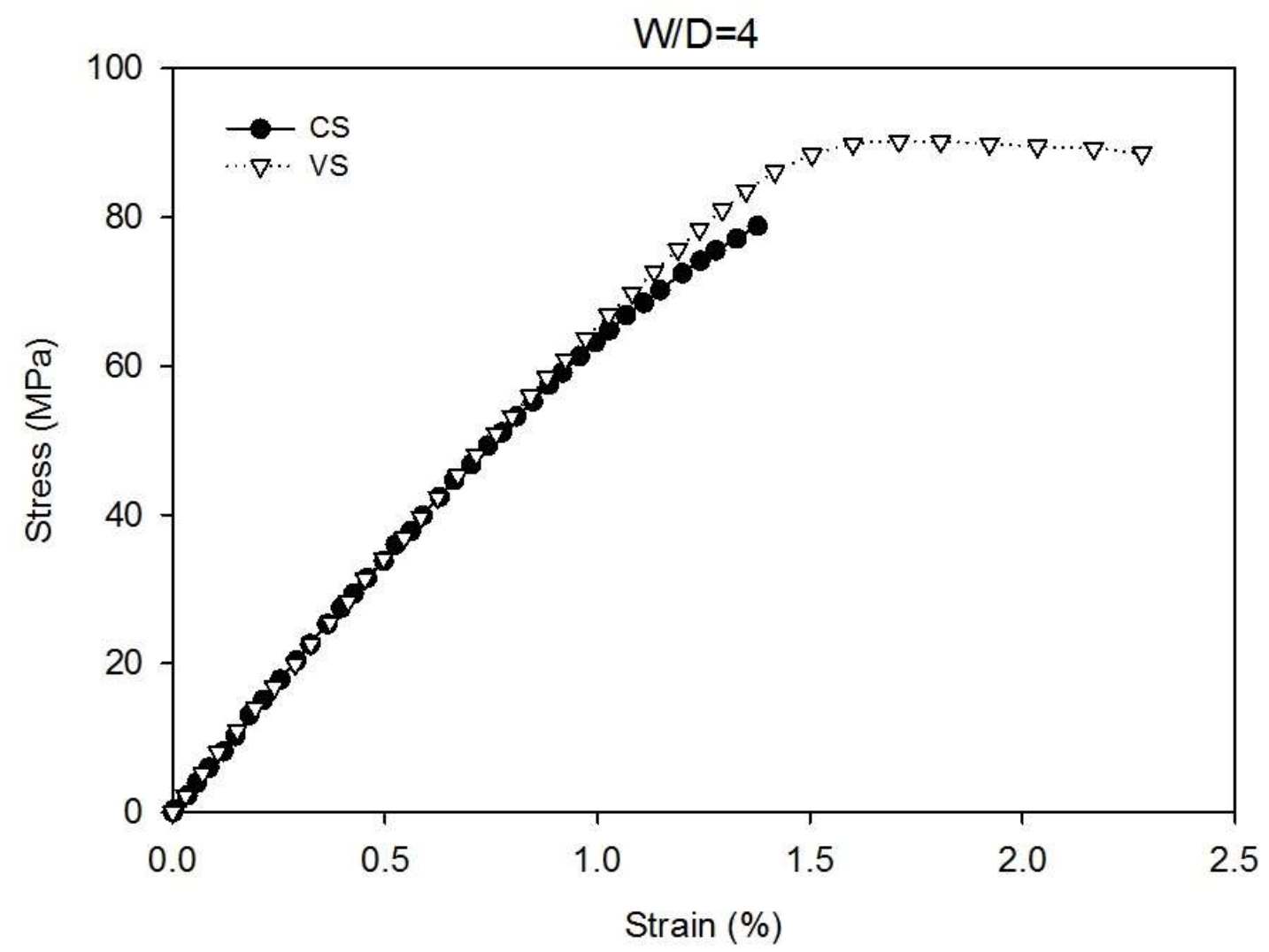

Figure 3.14. Stress-strain graph for CS and VS specimens with $w / D=4$.

As opposed to the straight rasters being cut at the hole, the curvilinear ones are continuous and transfer the load smoothly around the hole. For $w / D=2$, the net section is small, thereby the ratio of the curvilinear rasters to the straight ones is a large value. As a result, the equivalent stiffness of the VS specimens is larger compared to that of the CS specimens. Conversely, for $w / D=4$, the ratio of the curvilinear rasters to the straight ones is much smaller and thus the equivalent stiffness of the VS and CS samples are close. For $w / D=2$, since the maximum stress at the hole is high, when the straight raster layers fail, the curvilinear Vs layers also fail, resulting in a small improvement in the failure strain. On the other hand, for w/D=4, since the maximum stress at the hole is small, when the straight rasters fail, the curvilinear ones still can carry the load before reaching their yield limit; thus, increasing the failure strain of the CVS specimens compared to the CS ones. 
Table 0.2 summarizes ultimate strength and failure strain values obtained for open-hole tensile specimens with two configurations, $w / D=2$ and 4 . For a large hole $(w / D=2)$ configuration, $38.0 \%$ improvement in the mean ultimate strength is obtained using VS design, while improvement in the mean failure strain is only $4.0 \%$. For a small hole configuration $(w / D=4)$, improvement in tensile strength is $16.7 \%$, while failure strain is increased by $52.5 \%$ compared with CS design. For both cases, Coefficient of Variation (CV) is below 5\% for ultimate strength and it is below $10 \%$ for failure strain, which shows the reliability of the obtained results.

Table 0.2. Ultimate strength and failure strain for open-hole tensile specimens

\begin{tabular}{|c|c|cc|cc|}
\hline \multirow{2}{*}{ Sample } & \multirow{2}{*}{ Design } & \multicolumn{2}{|c|}{$\begin{array}{c}\text { Ultimate } \\
\text { Strength }\end{array}$} & \multicolumn{2}{c|}{ Failure Strain } \\
\cline { 3 - 6 } & & $\begin{array}{l}\text { Mean } \\
\text { (MPa) }\end{array}$ & CV & $\begin{array}{c}\text { Mean } \\
(\mathrm{mm} / \mathrm{mm})\end{array}$ & CV \\
\hline \multirow{2}{*}{ w/D=2 } & CS & 47.95 & $1.84 \%$ & 0.0125 & $8.97 \%$ \\
& VS & 66.19 & $3.96 \%$ & 0.0130 & $9.86 \%$ \\
\hline \multirow{2}{*}{ w/D=4 } & CS & 75.94 & $3.51 \%$ & 0.0141 & $6.83 \%$ \\
& VS & 88.63 & $4.53 \%$ & 0.0215 & $6.53 \%$ \\
\hline
\end{tabular}

Open-hole tensile strength of CS and VS design for the two configurations ( $w / D=2$ and 4) can be compared in Figure 3.15. Ultimate strength is calculated based on the gross cross-sectional area and the presence of the hole is disregarded. As a result, it is expected that the mean strength value increases by a reduction in the hole diameter (an increase in w/D parameter). It is also evident that for a larger hole diameter $(w / D=2)$, higher improvement in mean strength is achieved using VS design compared with CS design. This signifies the importance of Curvilinear Variable Stiffness (CVS) 3D printing, especially for high stress concentration design cases, that enables manufacturing lighter and cheaper products. 


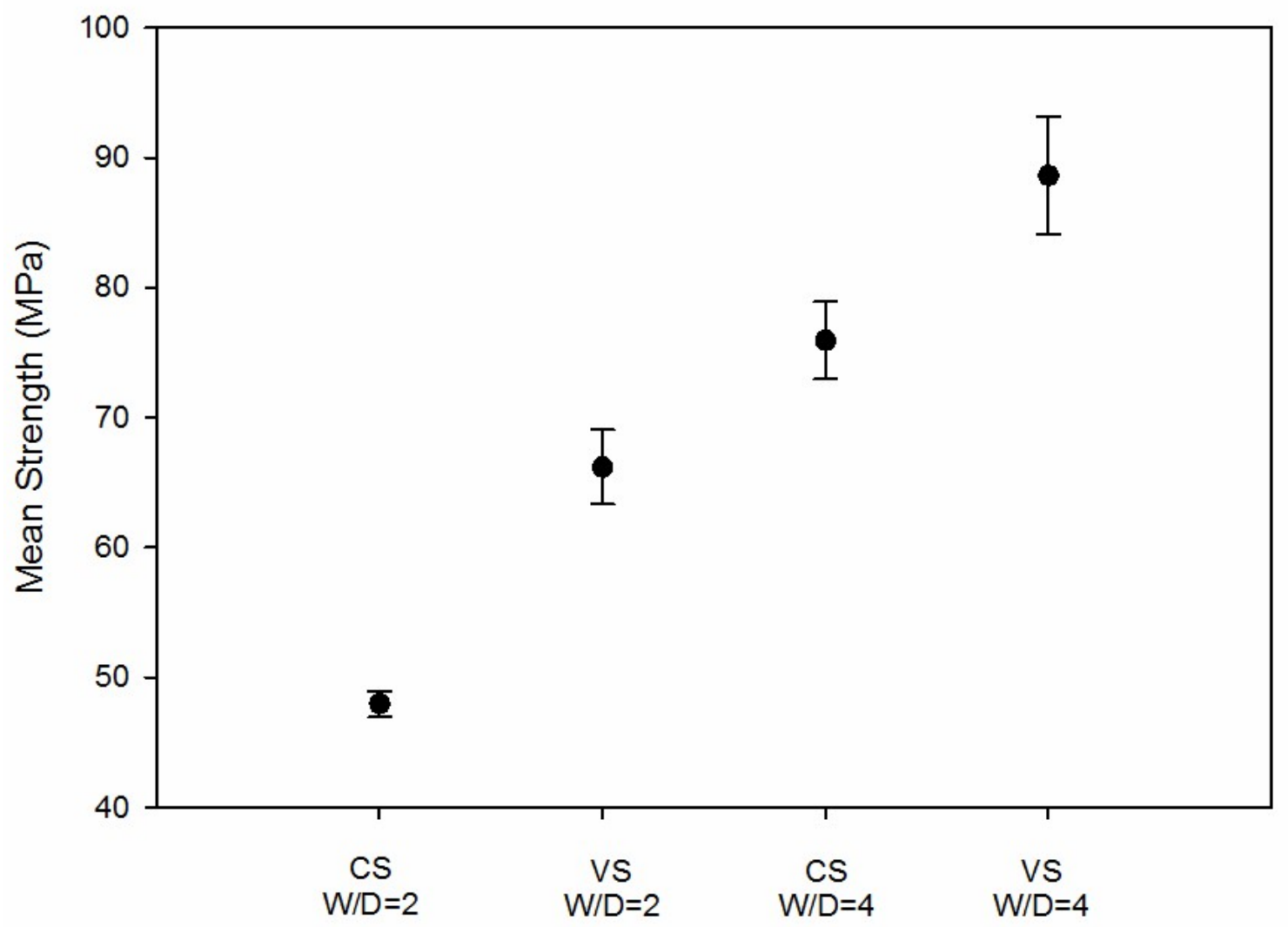

Figure 3.15. Mean strength for CS and VS designs for the two configurations ( $w / D=2$ and 4$)$.

\subsection{Conclusion}

Curvilinear Variable Stiffness (CVS) 3D printing has been explored to investigate potential improvements in open-hole tensile strength and failure strain of the specimens per ASTM D5766. An analytical approach has been described to find optimum curvilinear load paths using the analogy of field equations around a cylinder. For two hole diameters, Variable Stiffness (VS) and Constant Stiffness (CS) specimens have been designed and later manufactured using a commercial 3D printer. The Stress-strain graphs until failure have been created for the specimens, and ultimate strength and failure strain values have been calculated. We have observed that for the specimens with a larger hole diameter significant improvement in the ultimate strength is achieved, while the improvement in the failure strain is limited. On the other 
hand, for specimens with a smaller hole diameter, substantial improvement in failure strain is achieved, while the ultimate tensile strength improvement is less pronounced.

The results presented in this paper showed that 3D printing flexibility in manufacturing and anisotropic properties introduced by FDM can be utilized to improve structural performance of final parts. The approach of this study can be repeated with the use of highly directional materials as filaments in 3D printing, e.g. continuous carbon fibers. As a result, improvement in mechanical properties can be further increased and structural products for various applications across industries can be manufactured. 


\section{CHAPTER 4 CURVILINEAR VARIABLE STIFFNESS OF ARBITRARY CUT-OUT GEOMETRIES}

As previously shown with the open hole tension, potential functions are a capable means of effectively determining load paths around circular cut-outs. For these cut-outs, the load paths can be simply computed using the analytical solution to a cylinder in a uniform flow [13]. For more complex geometries, it is challenging to determine an analytical solution, as it may not exist. Therefore, for arbitrary geometries a numerical method, known as Source Panel Method, can be applied to determine the flow trajectories [18].

Fundamentally, the Source Panel Method is a finite element formulation in which the geometry is discretized by a finite number of flat panels. The superposition of all these elements would then allow for the generation of the load paths by interpolating over a mesh. Each element is known as a source panel, which emits a potential $Q[18]$.

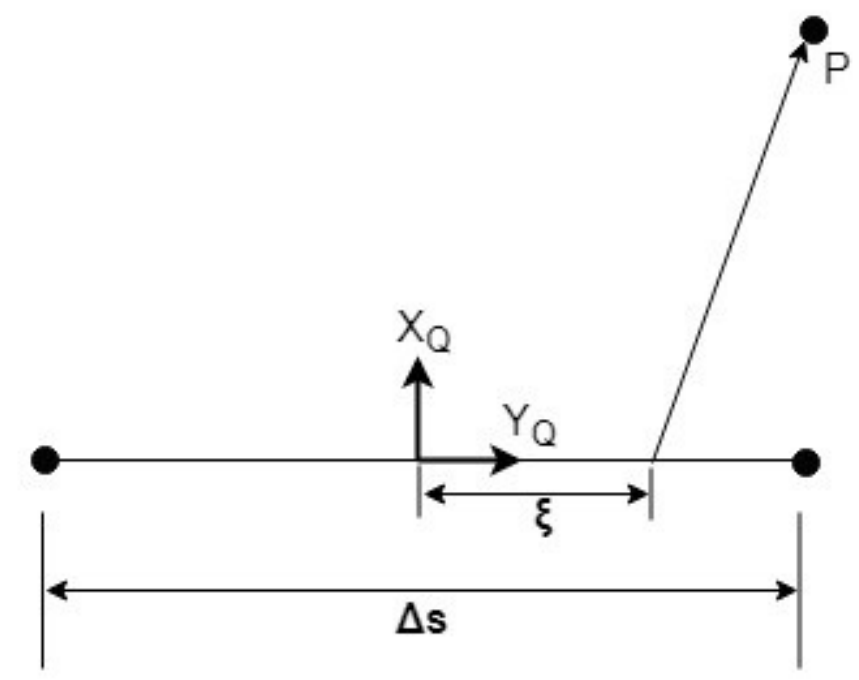

Figure 4.1. Schematic of a single panel in the $X_{Q} Y_{Q}$ refrence frame .

The components of the trajectory can be calculated at any point, $P$ using Eq. (1), where $X_{Q}$ and $Y_{Q}$ are the $\mathrm{x}, \mathrm{y}$ axis in the refrence plane of the panel of span $\Delta s$ and source strenght $Q$. For simplicity, the refrence point is always taken from the midpoint of the panel as seen in Figure 4.1. Since the contribution of the total panel is necessary, the integration of the total panel is taken with each arbritary distance along the panel represented by $\xi$ and the midpoint. The 
resulting components of the trajectory components are then given by $v_{Y_{Q}}$ and $v_{X_{Q}}$ oriented in the reference frame of the panel (Eqs (4.2) and (4.3)).

$$
\begin{gathered}
\phi_{P Q}=\int_{-\frac{\Delta s}{2}}^{\frac{\Delta s}{2}} \ln \sqrt{\left(x_{Q}-\xi\right)^{2}+y_{Q}^{2}} d \xi \\
v_{Y_{Q}}=\frac{\partial \phi_{\mathrm{PQ}}}{\partial y_{Q}}=Q \int_{\frac{\Delta s}{2}}^{\frac{\Delta s}{2}} \frac{y_{Q}}{\left(x_{Q}-\xi\right)^{2}+y_{Q}^{2}} \delta \xi=-\frac{1}{2} Q \ln \left(\frac{\left(x_{Q}+\frac{\Delta s}{2}\right)^{2}+y_{Q}^{2}}{\left(x_{Q}-\frac{\Delta s}{2}\right)^{2}+y_{Q}^{2}}\right) \\
v_{X_{Q}}=\frac{\partial \phi_{\mathrm{PQ}}}{\partial x_{Q}}=Q \int_{\frac{\Delta s}{2}}^{\frac{\Delta s}{2}} \frac{x_{Q}-\xi}{\left(x_{Q}-\xi\right)^{2}+y_{Q}^{2}} \delta \xi \\
=-Q\left(\tan ^{-1}\left(\frac{x_{Q}+\frac{\Delta s}{2}}{y_{Q}}\right)-\tan ^{-1}\left(\frac{x_{Q}-\frac{\Delta s}{2}}{y_{Q}}\right)\right)
\end{gathered}
$$

To transform from the $X_{Q}, Y_{Q}$ panel reference frame back to the global reference frame, a transformation function is used in Eq. (4.4), where $\theta_{x y}$ is the angle measured from the global $x$ axis. The inverse of this function can be used to transform into the panel reference frame.

$$
\overrightarrow{v_{x y}}=\left[\begin{array}{cc}
\cos \theta_{x y} & \sin \theta_{x y} \\
-\sin \theta_{x y} & \cos \theta_{x y}
\end{array}\right]\left[\begin{array}{l}
v_{X_{Q}} \\
v_{Y_{Q}}
\end{array}\right]
$$

The geometry of the cut-out can now be discretized as a series of $N$ panels as seen in Figure 4.2. A collocation point for each panel needs to be determined, which for simplicity as previously mentioned, is taken as the midpoint of each panel. At the collocation point of each panel, the normal and tangential vectors, $\widehat{n_{l}}$ and $\widehat{t}_{l}$, are found with respect to the global $X, Y$ system. 


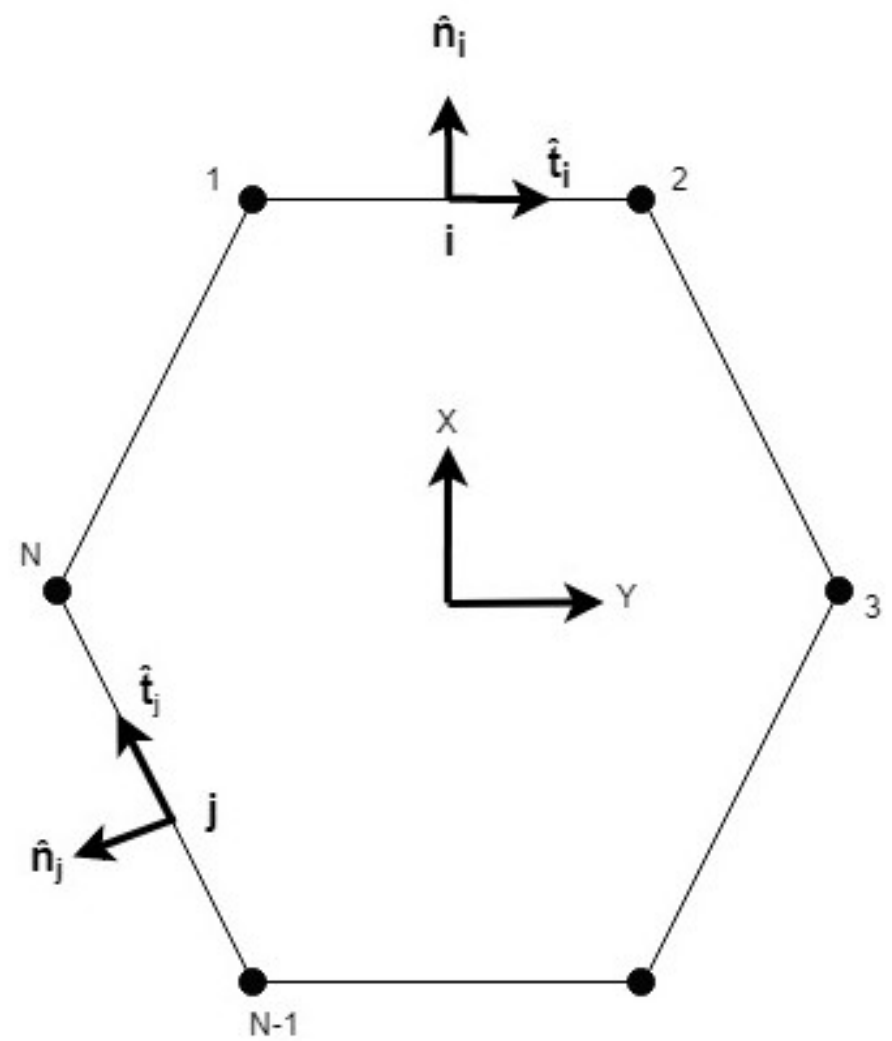

Figure 4.2. Discretized cut-out with Reference Frame

Since the superposition of the influences of each panel on others induces a trajectory, $v_{i j}$, at each corresponding panel, these influences need to be cancelled out in order to complete the boundary around the cut-out. To reduce the contributions of the trajectories to zero, the summation of all influences of the normal component of each panel and the summation of the ply angle, $U$, are taken in Eq. (4.5), where $\sigma_{j}$ is the source strength $Q$ of each panel and $\overrightarrow{v_{n_{l}}}$ is the total summation of the normal components of the trajectories at the collocation point of each panel.

The contribution, $\overrightarrow{v_{l \jmath}}$, is calculated using and (3), while transformation between reference frames is completed using Eq. (4.4).

Setting $\overrightarrow{v_{n_{l}}}$ to zero results in a system of equations with $N$ unknowns to solve for each $\sigma_{j}$ in Eq. (4.7). 


$$
\begin{gathered}
\overrightarrow{v_{n_{l}}}=\sum_{j=1}^{N} \sigma_{j}\left(\overrightarrow{v_{l j}} \cdot \widehat{n_{l}}\right)+\vec{U} \cdot \widehat{n_{l}}=0 \\
\sum_{j=1}^{N} \sigma_{j}\left(\overrightarrow{v_{l \jmath}} \cdot \widehat{n_{l}}\right)=-\vec{U} \cdot \widehat{n_{l}} \\
{\left[\begin{array}{ccc}
\overrightarrow{n_{1}} \cdot \overrightarrow{v_{11}} & \cdots & \overrightarrow{n_{1}} \cdot \overrightarrow{v_{1 N}} \\
\vdots & \ddots & \vdots \\
\overrightarrow{n_{N}} \cdot \overrightarrow{v_{N 1}} & \cdots & \overrightarrow{n_{N}} \cdot \overrightarrow{v_{N N}}
\end{array}\right]\left[\begin{array}{c}
Q_{1} \\
\vdots \\
Q_{N}
\end{array}\right]=\left[\begin{array}{c}
-\overrightarrow{n_{1}} \cdot \vec{U} \\
\vdots \\
-\overrightarrow{n_{N}} \cdot \vec{U}
\end{array}\right]}
\end{gathered}
$$

Once the panel strength values are known, the trajectory of any arbitrary point can be found as the summation of influences of each panel on that point plus the contribution from the ply angle as seen in Eq. (4.8), where $\overrightarrow{v_{x y_{Q_{l}}}}$ is the trajectory induced at point $(x, y)$ by panel $Q_{i}$ and $v_{P}$ is the trajectory at that point.

$$
v_{P}=\sum_{i=1}^{N} \overrightarrow{v_{x y_{Q_{l}}}}+\vec{U}
$$

\subsection{Curvilinear Path Generation}

A MATLAB script was developed for calculating the trajectories. The inputs were the initial ply angle and a matrix that contains the locations at which the fiber direction is to be computed at. The cut-out was discretized into 100 source panels as shown in Figure 4.3, after which the normal and tangential vectors were determined at the collocation point of each panel as seen in Figure 4.4. Once this was completed, the $\sigma_{j}$ matrix was determined to evaluate the strength value of each panel and the trajectory can be calculated at each element centroid. Examples of the 
load paths generated around the cut-out can be seen in Figure 4.5 which were generated by first creating a numerical mesh-grid of numerical points at which the local angles are computed. The local angles are then integrated across from a starting point and direction to generate streamlines.

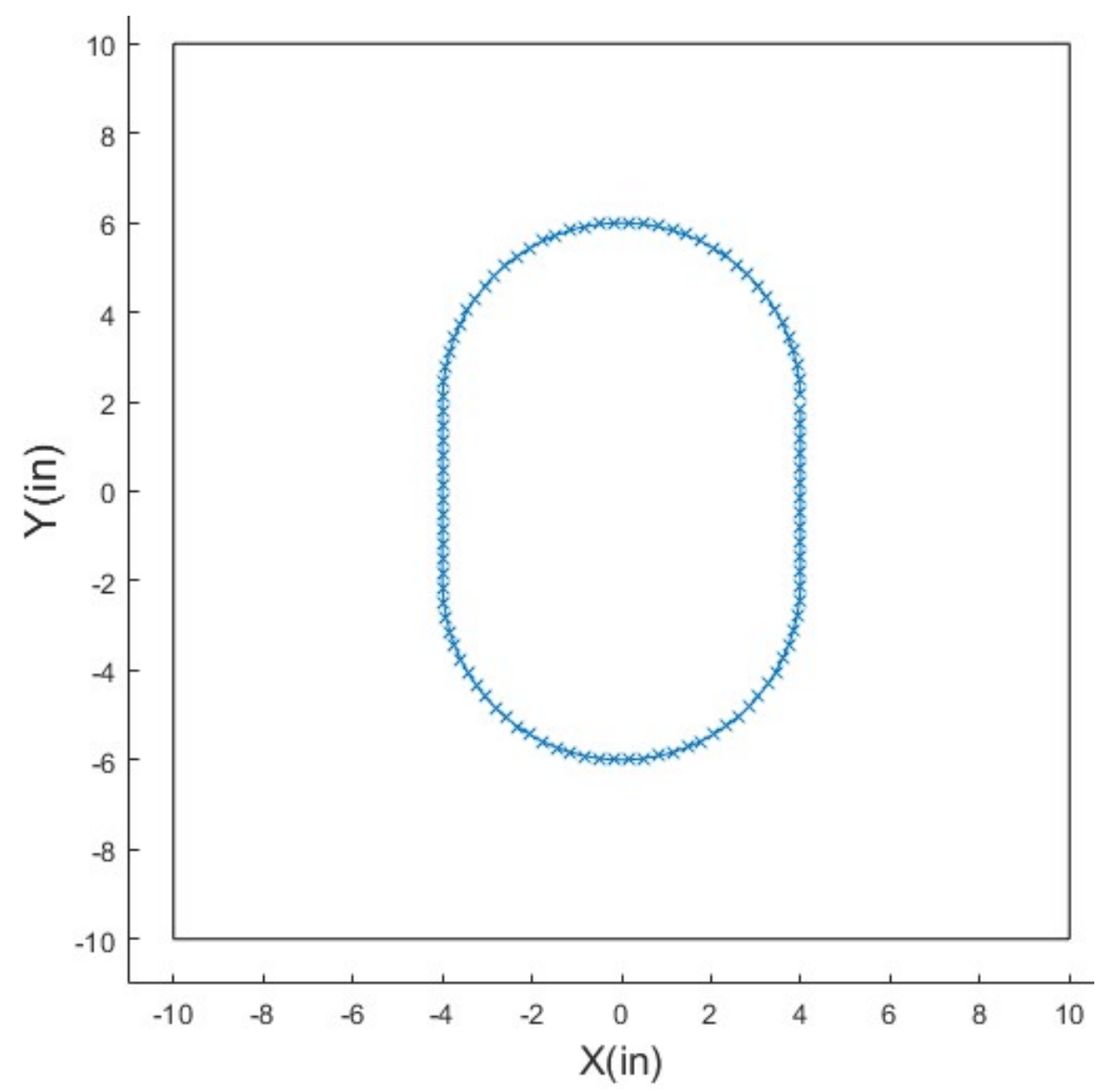

Figure 4.3. Discretized fuselage cut-out showing panels and collocation points. 


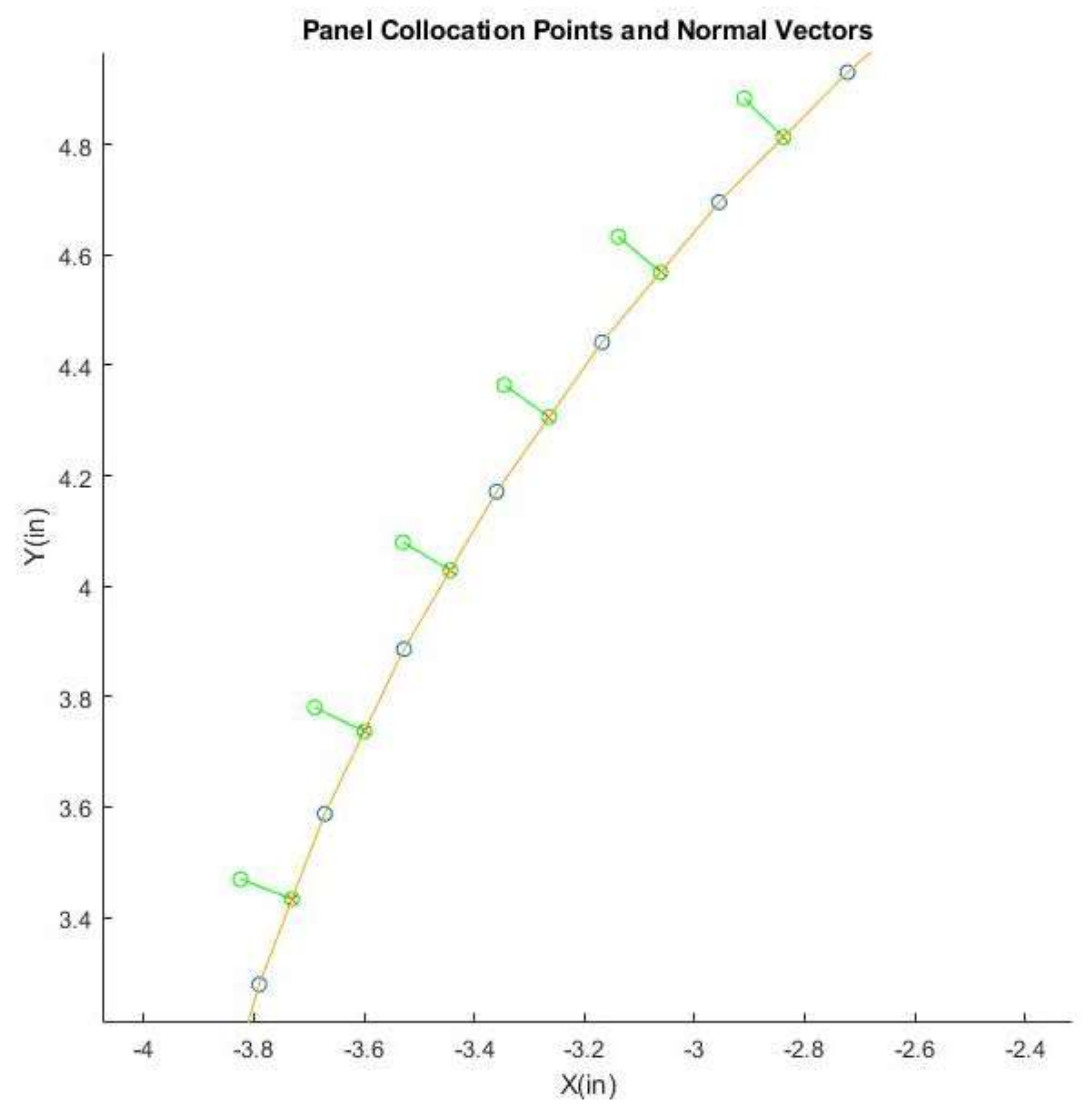

Figure 4.4. Normal and Tangential vectors plotted. 


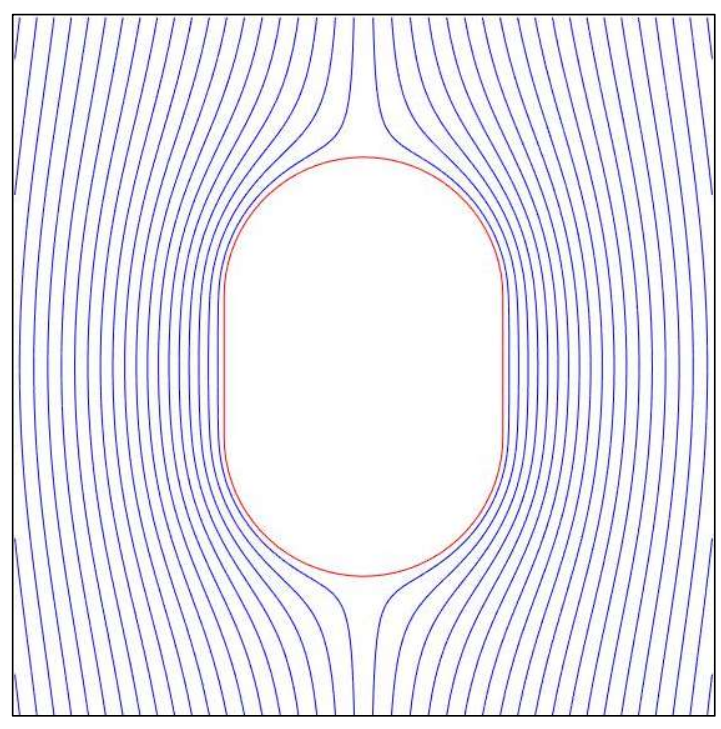

(a)

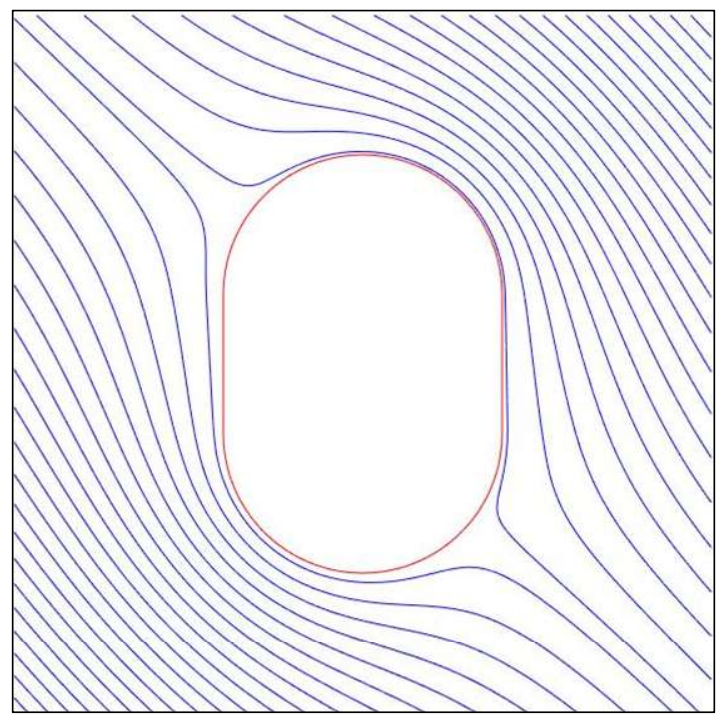

(c)

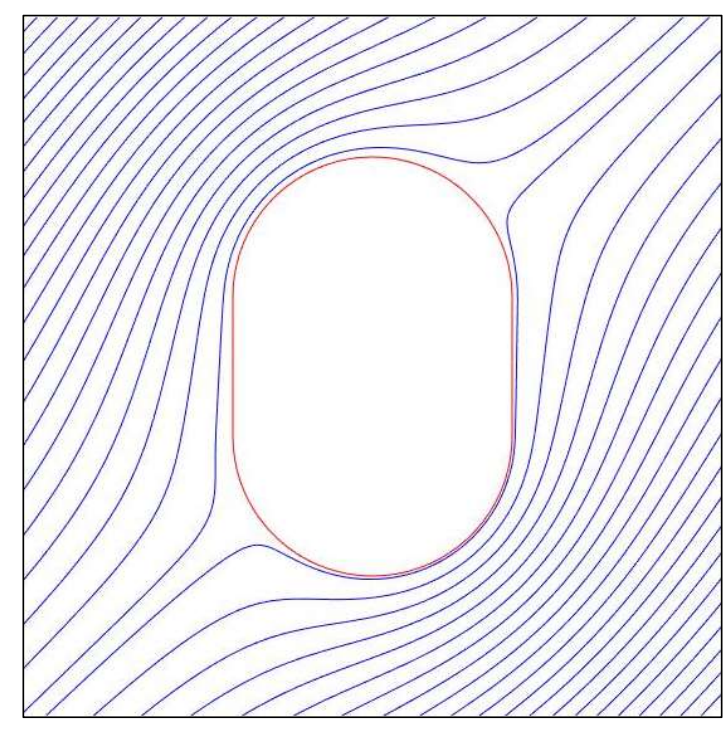

(b)

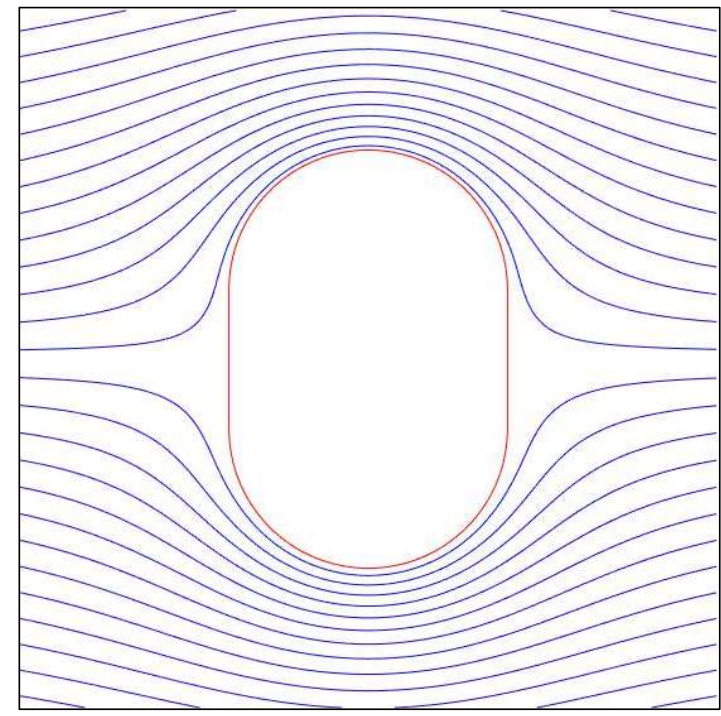

(d)

Figure 4.5. Examples of load path plotted at various angles: (a) $90^{\circ}$; (b) $45^{\circ}$; (c) $-45^{\circ}$; (d) $0^{\circ}$.

In a Finite Element (FE) model for a constant-stiffness composite laminate, where the fibers are straight and their orientations are constant within each ply, a single property set with a specified layup is enough to define the material properties for all elements. However, in a variable stiffness design, the fiber orientation is varying spatially meaning each element may have a different fiber orientation and layup. Since the fiber path is discretized by the mesh size, the 
fiber orientation and the layup for each element needs to be recalculated. Therefore, MATLAB subroutines are developed that connect the FE solver to the fiber path generator. At each step, the subroutine gets the mesh data from the FE solver, extracts the element centroids and transfer them to the path generator code, which calculates the fiber angle in each element based on the Source Panel Method previously described. Having the fiber angle at each element and assuming the fiber angle is straight within each element, the MATLAB subroutines then update the PCOMP card (layered shell property), defining the element layup in NASTRAN finite element input file. Since the ply angle within each element can be different for each ply, this process must be repeated through the thickness. The geometry is discretized with a mapped mesh using CQUAD4 (Quadrilateral Plate) elements and a convergence study is performed to ensure an enough refined mesh is used in the study.

To evaluate the performance of the design, the first ply failure is considered by calculating the Failure Indices (FI) using Tsai-Wu criteria. It should be recalled that for a VS design, the fiber angle is changing within each ply meaning that failure index will be different for each element. As a result, FI values varies spatially and it is required to inspect all elements to determine the critical failure index for the whole laminate.

\subsection{Case Study}

A window opening in a pressurized fuselage skin modeled as a plate with a cut-out located at the plate center is considered as a case study (Figure 6). As suggested by Alhajahmad et al. [17], the applied pressure $(P)$ is translated to axial $\left(F_{x}\right)$ and hoop $\left(F_{y}\right)$ tensile loads, calculated as:

$$
F_{x}=\frac{P R_{f}}{2} b \quad F_{y}=P R_{f} a
$$

, where $\mathrm{a}$ and $\mathrm{b}$ are the length and the width of the plate, respectively. The internal pressure is $\mathrm{P}$ $=15 \mathrm{psi}$, the length and width are $\mathrm{a}=\mathrm{b}=20 \mathrm{in}$, and the fuselage radius is $R_{\mathrm{f}}=100$ in [5].

All the exterior edges are constrained with a simply support boundary conditions and the internal opening edges are free. In addition to the internal pressure, an external compressive force of $F_{x}{ }^{B}$, 
representing the contribution from fuselage bending, is applied to the side edges. It is worth mentioning that the goal of this study is to demonstrate the effectiveness of CCVS design with the fiber path defined using Source Panel Method; therefore, the interactions from adjacent fuselage panels are ignored for the sake of simplicity.

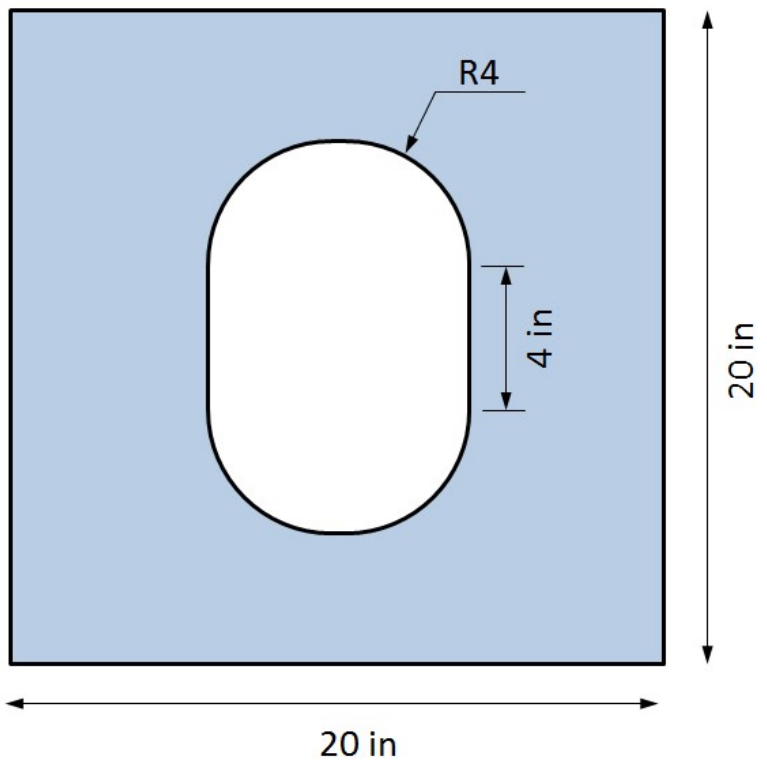

(a)

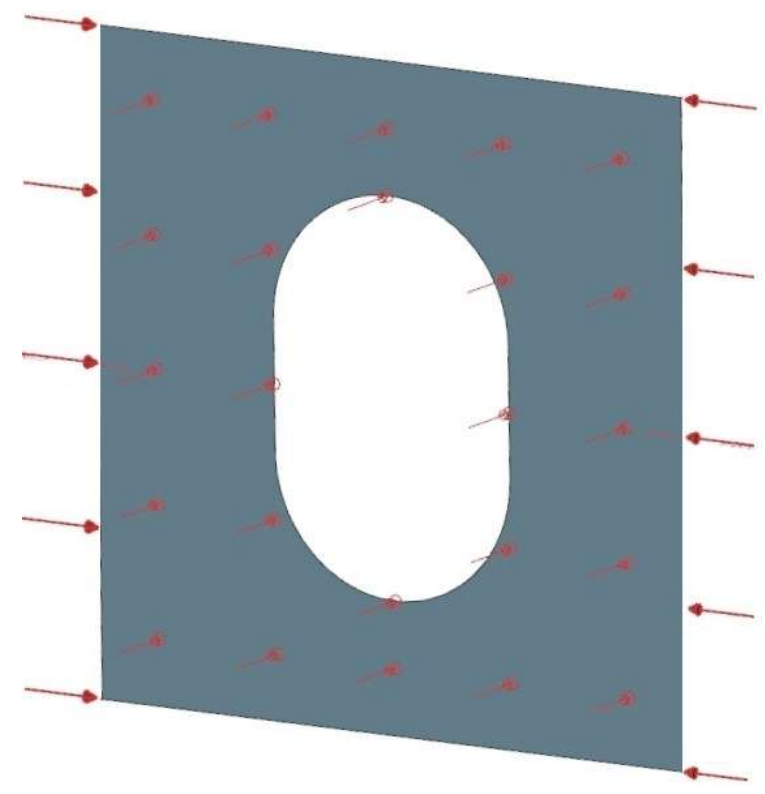

(b)

Figure 4.6 Case study: (a) schematic of the plate with a central cut out; (b) applied loading (Pressure and external compressive force) where the exterior edges are simply supported and the internal opening edges are free.

It is assumed that the plate is flat, 16 layer, balanced, and symmetric laminate with a variable-stiffness layup $\left[ \pm \mathrm{CCVS}_{1} / \pm \mathrm{CCVS}_{2} / \pm \mathrm{CCVS}_{3} / \pm \mathrm{CCVS}_{4}\right]_{s}$ made of the material properties presented Table 4.1. A quasi-isotropic laminate, $[45 / 0 /-45 / 90]_{2 s}$, with the same material is considered as the baseline for analysis. 
Table 4.1 Material properties [17]

\begin{tabular}{ccc}
\hline Material Properties & \multicolumn{2}{c}{ Values } \\
\hline $\mathrm{E}_{1}$ & 30 & $\mathrm{msi}$ \\
$\mathrm{E}_{2}$ & 0.75 & $\mathrm{msi}$ \\
$\mathrm{V}_{12}$ & 0.25 & \\
$\mathrm{G}_{12}$ & 0.375 & $\mathrm{msi}$ \\
$\mathrm{X}_{\mathrm{t}}$ & 150 & $\mathrm{ksi}$ \\
$\mathrm{Y}_{\mathrm{t}}$ & 6 & $\mathrm{ksi}$ \\
$\mathrm{X}_{\mathrm{c}}$ & 100 & $\mathrm{ksi}$ \\
$\mathrm{Y}_{\mathrm{c}}$ & 17 & $\mathrm{ksi}$ \\
$\mathrm{S}$ & 10 & $\mathrm{ksi}$ \\
\hline
\end{tabular}

\subsection{Optimization}

An important benefit of the proposed Source Panel Method is that it offers the great flexibility of placing the material along a curvilinear path with only one design variable, i.e. Initial Ply Angle (IPA), for each ply. Therefore, similar to a straight fiber layup, there will be one design variable for each ply angle resulting in an equal computational cost of optimization for both variable stiffness and straight fiber cases. This benefit will be significant especially for designing large and thick laminates.

Here, the maximum strength or minimum failure index of the laminate is considered as the objective function. The goal is to find IPAs (design variables) that provide the minimum failure index. Therefore, the optimization problem can be written as follows

$$
\begin{aligned}
& \min _{\mathbf{x}}\{\operatorname{FI}(\mathbf{x})\} ; \mathbf{x}=\left(T_{1}, T_{2}, T_{3}, T_{4}\right)^{T} \\
& \text { s.t. }\left\{T_{1}, T_{2}, T_{3}, T_{4} \in\left[-90^{\circ},+90^{\circ}\right]\right\}
\end{aligned}
$$

Where $\mathbf{x}$ is the vector of design variables, i.e., $T_{1}$ to $T_{4}$ that are the IPAs for each of distinct plies, respectively; and FI represents the failure index. It is worth to mention the design variables 
should be integers to respect the manufacturing accuracy of a typical AFP for placing the fiber in a specified angle. Since the fiber angle is changing along a curvilinear path, the relation between the design variables, i.e. ply angles, and the objective function, i.e. the failure index, is a nonlinear complex function that needs to be calculated via FEM. It has been shown that the structural response of a composite laminate when it is a function of ply angle has many local optima [58] [59]. As a result, evolutionary strategies like Genetic Algorithm (GA) have been widely used and recommended for optimizing laminated composites [60] [61]. However, GA is a population-based algorithm requires a large number of function evaluations to reach the optimum solution. To improve the efficiency of the optimization process, a surrogate-based algorithm suggested By Arian Nik et al. [62] is used in this study.

\subsection{Results and discussions}

In terms of FE modeling, the entire plate is modeled, rather than just a quarter of the plate. The reason is that for any angle except $0^{\circ}$ and $90^{\circ}$, the design is not symmetric with respect to the $\mathrm{Y}$-axis (Figure 5). A quasi-isotropic laminate is considered as the reference baseline design and the optimization problem is solved to obtain a VS design. The optimum design for maximum failure index is found as $[ \pm 1 / \mp 87 / \pm 88 / \pm 1]_{s}$, which has a failure index of 0.29 compared to 0.68 for the quasi-isotropic laminate. It shows that a CCVS design can significantly increase the failure strength of the design by $57 \%$. Figure 4.5 shows the fiber paths for the optimum CCVS laminate.

Figure 7 shows the explored designs during the optimization process, where each line represents a design and the final design is marked with a thick line. It can be seen that many of explored designs are near the final optimum design, i.e. the angle for $\mathrm{CCVS}_{1}$ and $\mathrm{CCVS}_{4}$ is $1^{\circ}$, the angle for $\mathrm{CCVS}_{2}$ and $\mathrm{CCVS}_{3}$ is $-87^{\circ}$ and $+88^{\circ}$, respectively. It means that the last iterations of the optimization tried to fine tune the ply angle within a few degrees range that shows the stability of the final design. 


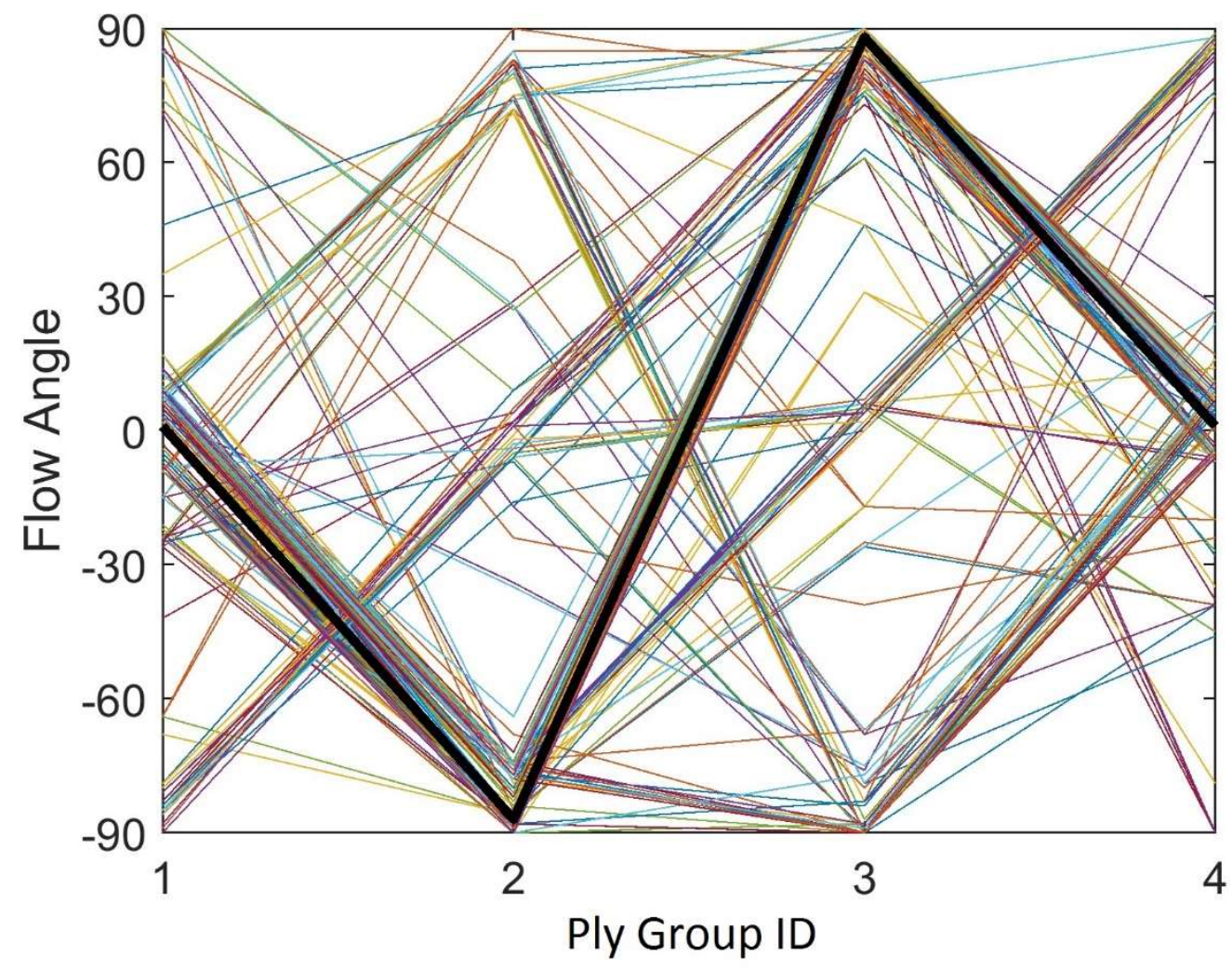

Figure 4.7 Explored designs during the optimization.

Figure 4. and Figure 4. show the stress contours and failure index plots for the quasiisotropic and the optimum CCVS designs, respectively. It should be noted the plots show the envelope of the results over all plies. It can be seen that compared to the quasi-isotropic design, the CCVS design reduced the normal stresses $\sigma_{x x}$ and $\sigma_{y y}$ all over the plate especially around the cut-out, thereby increasing the strength of the panel. The $\sigma_{x y}$ in the CCVS design is $15 \%$ higher than that in the quasi-isotropic design. Comparing the failure index plots (Figure 4.d and Figure 4.d), it can be seen that the CCVS design generally redistributes the stress all over the panel and there is much less stress gradient compared with the quasi-isotropic design. Therefore, it can be concluded that a CCVS design allows for a significant reduction in the stress concentration around the geometrical discontinuities like cut-outs. 


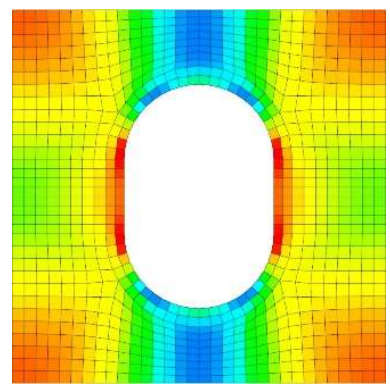

a) $\sigma_{x x}(p s i)$

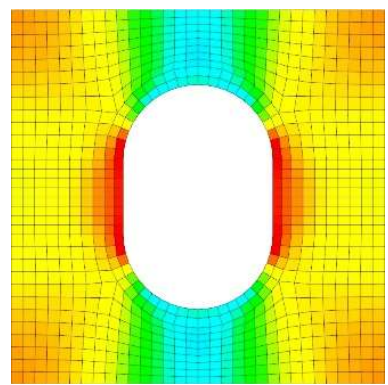

b) $\sigma_{y y}(p s i)$

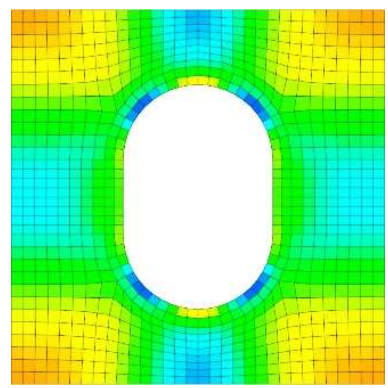

c) $\sigma_{x y}(p s i)$

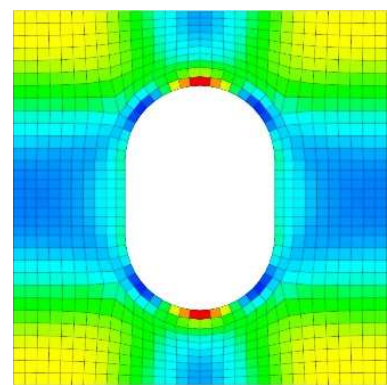

d) Failure Index

Figure 4.8 Envelope of stress distribution and failure index plots for the quasi-isotropic design.

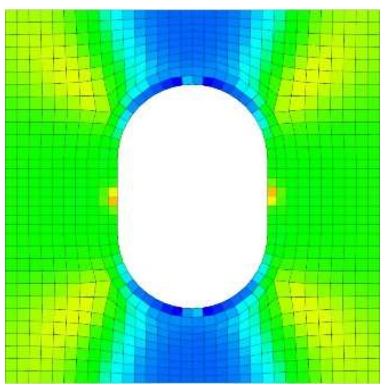

a) $\sigma_{x x}(p s i)$

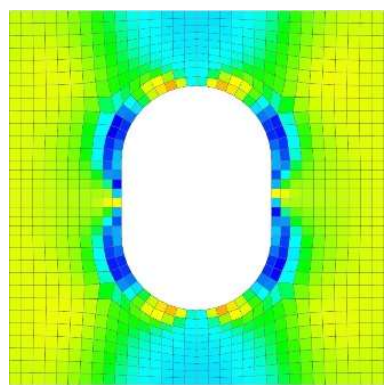

b) $\sigma_{y y}(p s i)$

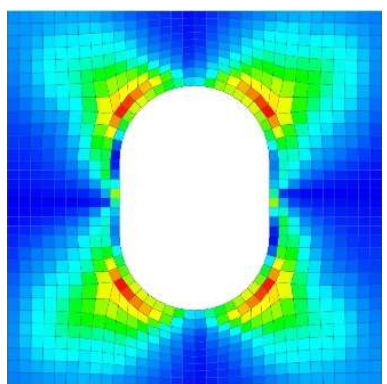

c) $\sigma_{x y}(p s i)$

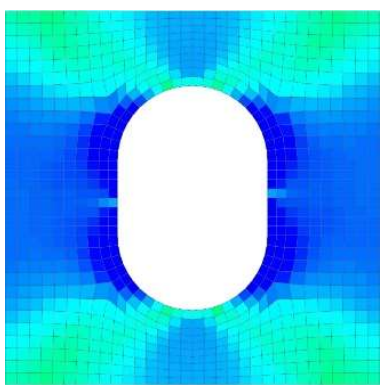

d) Failure Index

Figure 4.9 Envelope of stress distribution and failure index plots for the optimum CCVS design 
A closer look at the optimum design $[ \pm 1 / \mp 87 / \pm 88 / \pm 1]_{\mathrm{s}}$ and the fiber trajectories depicted in Figure $4.5 \mathrm{~d}$ shows that for the exterior plies with an IPA of close to $0^{\circ}$, there is a relatively sharp change of the fiber angle around the centerline of the opening (see Figure $4.5 \mathrm{~d}$ ). It causes the local regions to be relatively less stiff compared to the quasi-isotropic design, thereby relieving stresses. This is evident in the total displacement distribution plots for the CCVS compared with the quasi-isotropic design in Figure 4., where there are bulging towards the cut-out.

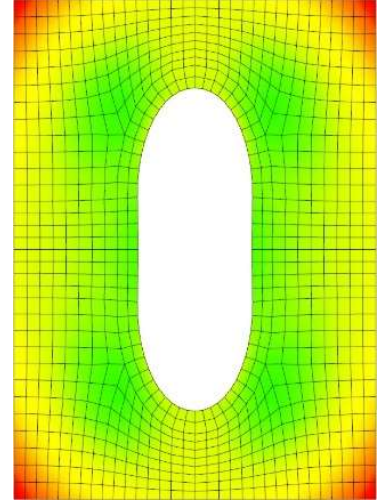

(a)

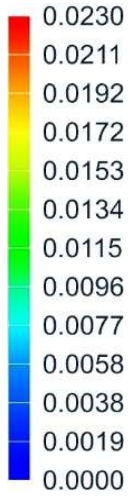

0.0000

Figure 4.10 Total displacement distribution plots (deformed shape with a factor of 100); (a) quasi-isotropic laminate; (b) CCVS design.

Another aspect of the CCVS design is that the failure scatters over a ply and through the thickness in contrast with the straight fiber design where a whole ply fails as seen in Figure 4.. It will potentially improve crash worthiness of the design as the laminate might withstand much more load, which can be evaluated by a future studies using a progressive failure analysis.

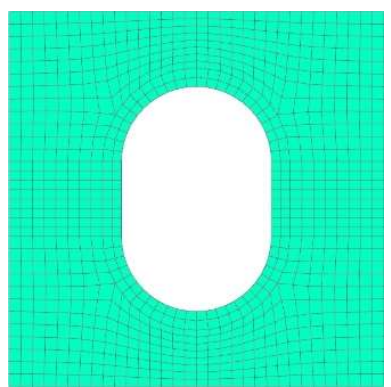

(a)

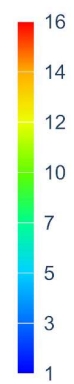

Figure 4.11 Layer number with the maximum failure index: (a) Quasi-isotropic laminate; and (b) CCVS design.

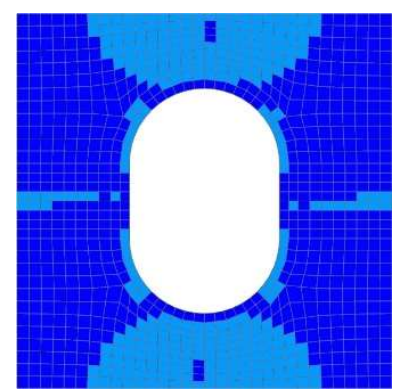

(b) 


\subsection{Conclusion}

The CCVS design was carried out by defining fiber trajectories using the Source Panel Method. It was shown that the proposed trajectory formulation can represent the CCVS design for each ply with only one design variable, which reduces the computational cost of optimizing a CCVS design to that of a conventional constant-stiffness design. It was also demonstrated that the optimal CCVS design can improve the first ply failure strength of the design by $57 \%$. Furthermore, the CCVS design has the benefit of distributing the failure over the laminate and potentially increate the last ply failure strength of the design.

Future work is required to study potential improvements from CCVS under off-design conditions. Multi-directional loading, the addition of $\pm 45^{\circ}$ layer to the VS layup, and the buckling performance as well. In addition, the progressive failure analysis of a CCVS design can be completed to explore the full potential of this design. Continuous Tow Shearing (CTS) and additive manufacturing of continuous carbon filaments can be used to fabricate CCVS designs. Certain defects, gaps and overlaps, will emerge during manufacturing and future work will also include modeling defects in the FEM using Defect Layer Method. Furthermore, to validate the feasibility of CCVS, panels will have to be physically manufactured and tested. The methodology to potentially manufacture CCVS designs are described in the following sections. 


\section{CHAPTER 5 INDUSTRIAL ROBOT 3D PRINTING USING FUSED FILAMENT FABRICATION (FFF)}

Conventional 3D printing with thermoplastic polymers using an FFF process are limited in the 3-axis motion due to a fixed gantry system. Furthermore, these systems can be considered as having only 2.5 axis of travel, as the $z$ axis is typically only used to lift the nozzle up to the height of the next layer. Multi-axis deposition has seen limited application in 3D printing but has been recently been of interest to save material and deposition time.

A 3D printing industrial robot work cell was developed for the purposes of multi axis deposition of thermoplastic. The end goal is continuous fiber deposition, which can be considered a form of AFP. Many of the mechanisms and tool motions for AFP and FFF are fairly similar. Therefore, using FFF with thermoplastic was a good surrogate to prototype future fiber deposition strategies. Using thermoplastic also allowed for inexpensive experimentation while going through the learning curve of programming the industrial robot.

An industrial robot was chosen for the purposes of FFF over designing a custom 5 axis gantry for a number of reasons. The key motivation was that an adequately sized robot capable of 6 degrees of freedom could be purchased off the shelf with all the support and software already commercially developed. This support and simplicity would reduce the time to completion of the project as the need to develop the motion control system and gantry are completely removed. The simplicity does come at a cost however. Compared to a gantry system, the industrial robot will be much less rigid and provide less accuracy as the linear movements need to be interpolated through all 6 degrees of freedom. 


\subsection{Work Cell development}

The industrial robot of choice was an ABB IRB1200 with an IRC5 controller. The reach of the robot was $900 \mathrm{~mm}$ with a $5 \mathrm{Kg}$ payload. The IRB1200 features 6 degrees of freedom as well as multiple options of connectivity such as Serial, TCP/IP and MODBUS.

A workcell was built around the IRB1200 for safety purposes. The robot was affixed to an existing table and an enclosure was built around it.
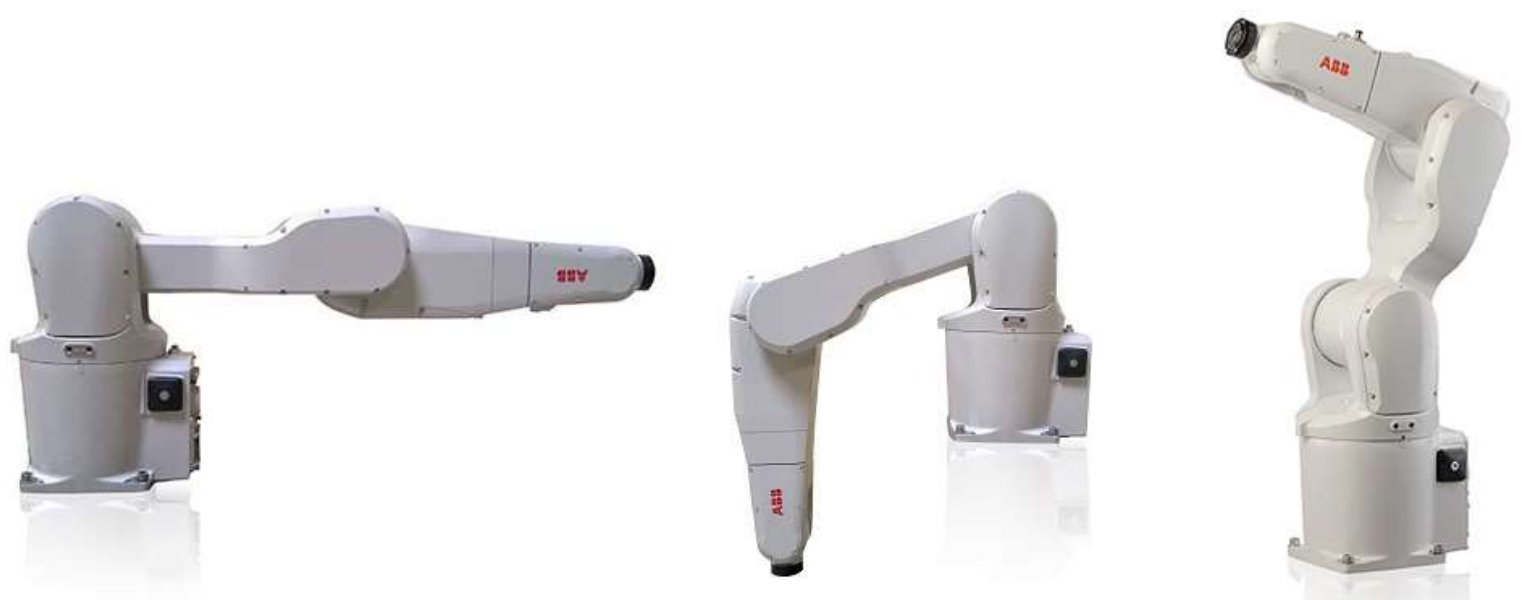

Figure 5.1 ABB IRB1200 Industrial Robot demonstrating degrees of freedom 


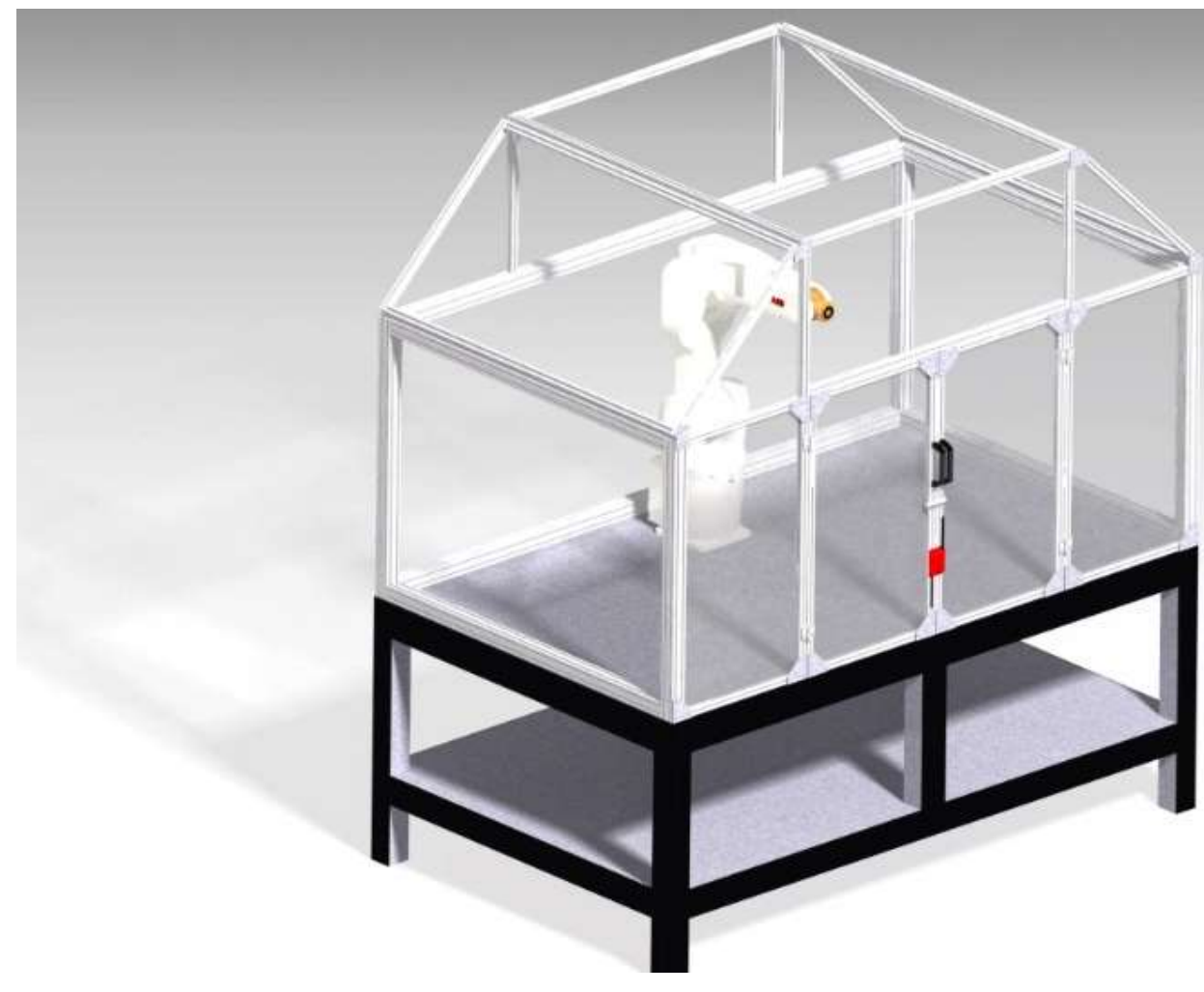

Figure 5.2 Render of IRB1200 Enclosure

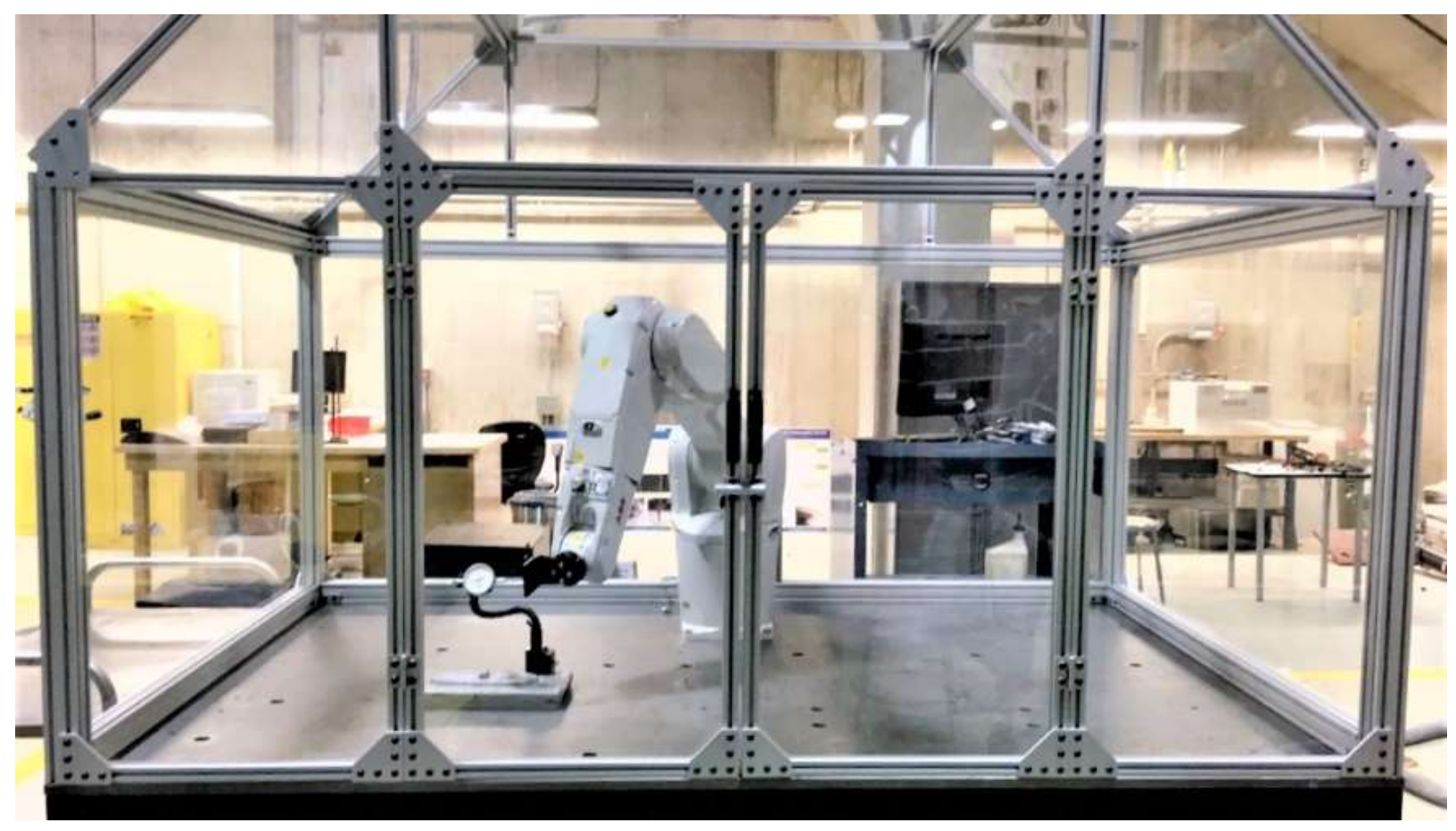

Figure 5.3 Completed Workcell 


\subsection{D Printing Integration}

An E3D Titan Aero hot end extruder combo was integrated onto the IRB1200. This hot end was chosen for its compact design, low weight, and reliable feeding characteristics. The material that was used for prototyping was Poly Lactic Acid (PLA). PLA features a low glass transition temperature and inexpensive cost, which allows for prototyping robot motion techniques without fear of overrunning costs in the process. A $1 \mathrm{Kg}$ spool of PLA was mounted on the back of the enclosure and fed through a Teflon tube that was affixed the various mounting points provided on the IRB1200.

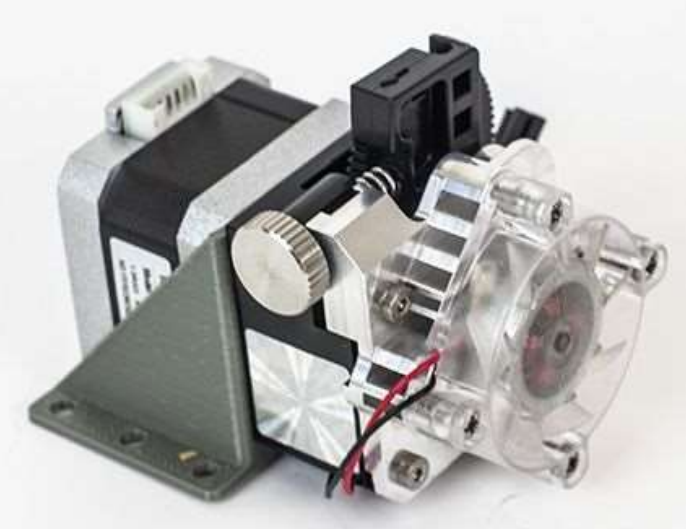

Figure 5.4 Picture of an E3D Titan Aero hotend

A 24 in by 24 in heated aluminum build plate was affixed to the workcell. The build plate was mounted onto a tapped work holding table.

The IRC5 controller is a highly capable controller which features many capabilities that are useful in an industrial setting. The language used by the controller is a proprietary ABB language known as RAPID. RAPID is capable to both programing the motion of the robot as well as being a fully featured object-oriented language that is similar in syntax to JAVA. The RAPID code is run on the ABB proprietary operating system called Robot Ware. 
Although the IRC5 controller is unable to run a third party external axis directly, it is possible to interface them through its many peripheral interfaces. Rapid code can be written and subroutines can be developed for this explicit purpose. At any point in the robot motion, the function can be called to run a separate task. For the vast majority of FFF extruders, a stepper motor is required to deposit the filament. A certain amount of filament must be deposited per distance traveled by the Tool Center Point (TCP), which correlates to a certain number of steps.

To address the need for a system to drive the stepper motor, a Programmable Logic Controller (PLC) box was designed to drive the stepper motor. The system is connected to the IRC5 controller through a Serial connection using the RS232 protocol. Before the beginning the robot motion a function called Extruder is called with the amount of filament to be deposited in millimetres. Once the amount of filament to be deposited by the extruder is received, the distance is converted into stepper motor pulses and deposition begins. 


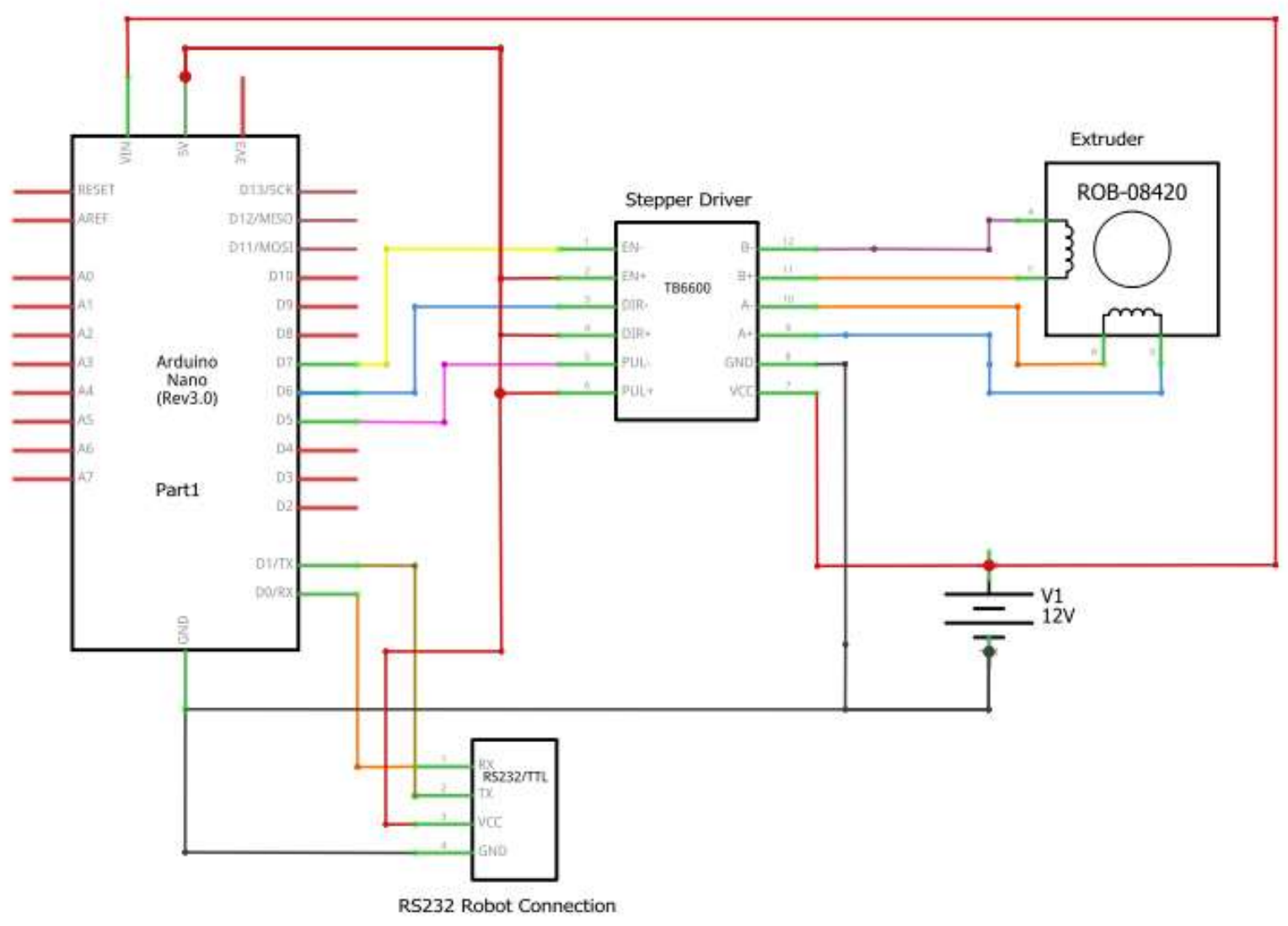

Figure 5.5 Electrical Schematic of Stepper motor driver

A second PLC box was designed to control the temperature of the hot-end using an industrial temperature controller and relay circuit. The PLC box was separately controlled and did not receive any input from the IRC5 controller. The power input was from the $120 \mathrm{~V}$ wall outlet and the temperature was set before a print could begin. 


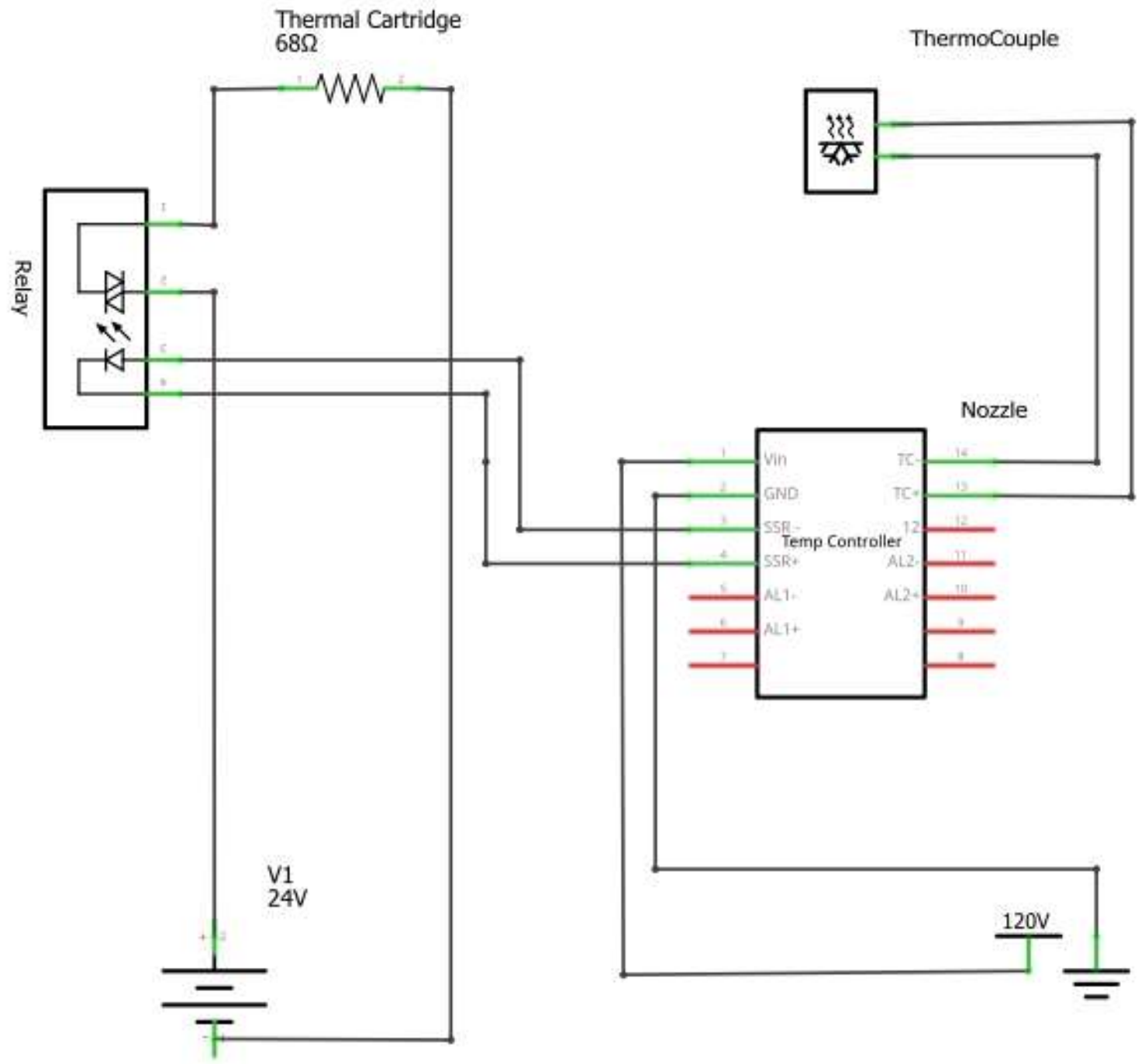

Figure 5.6 Electrical system of Temperature controller 


\subsection{Offline Programming}

An Offline Programming (OLP) tool called ROBODK was used for post processing and generating the robot paths. ROBODK includes and Forward and Inverse Kinematics solver that is accessible through the GUI and the included API. Several toolboxes and postprocessors are available for a variety of industrial robots. A very useful toolbox included in ROBODK is the GCODE to robot path converter that is capable of importing GCODE generating by any CAM software and convert into the motion paths that can be modified as needed in ROBODK. This powerful tool allows GCODE from a 3D printer slicer to be used on an industrial robot.

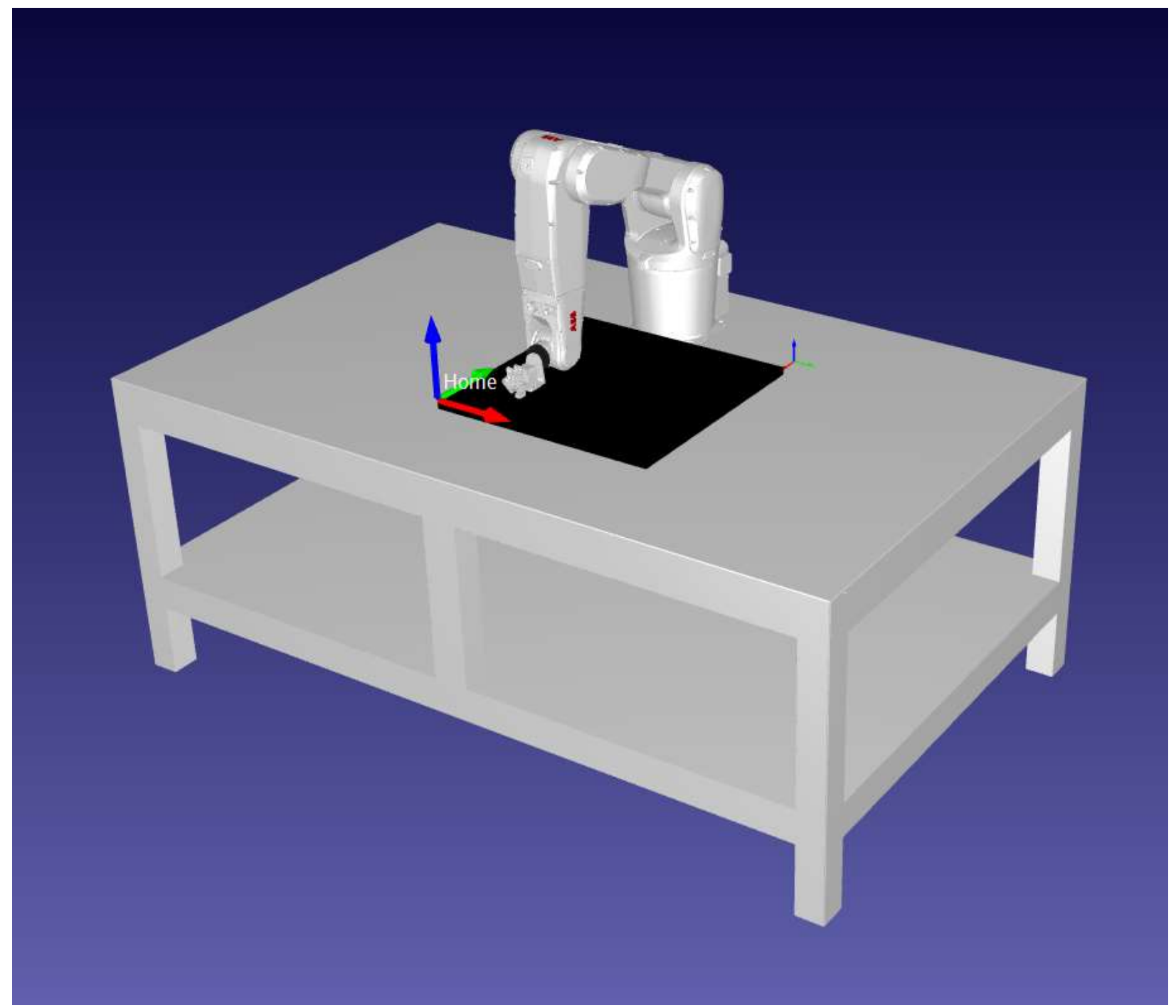

Figure 5.7 Virtual Workcell in ROBODK with build plate 
A virtual workcell was generated in ROBODK to accurately represent the physical setup. The E3D Titan Aero hot end was also imported into ROBODK to determine the Tool Center Point (TCP). For best results, the virtual positions need to be adjusted slightly to account for real world discrepancies.

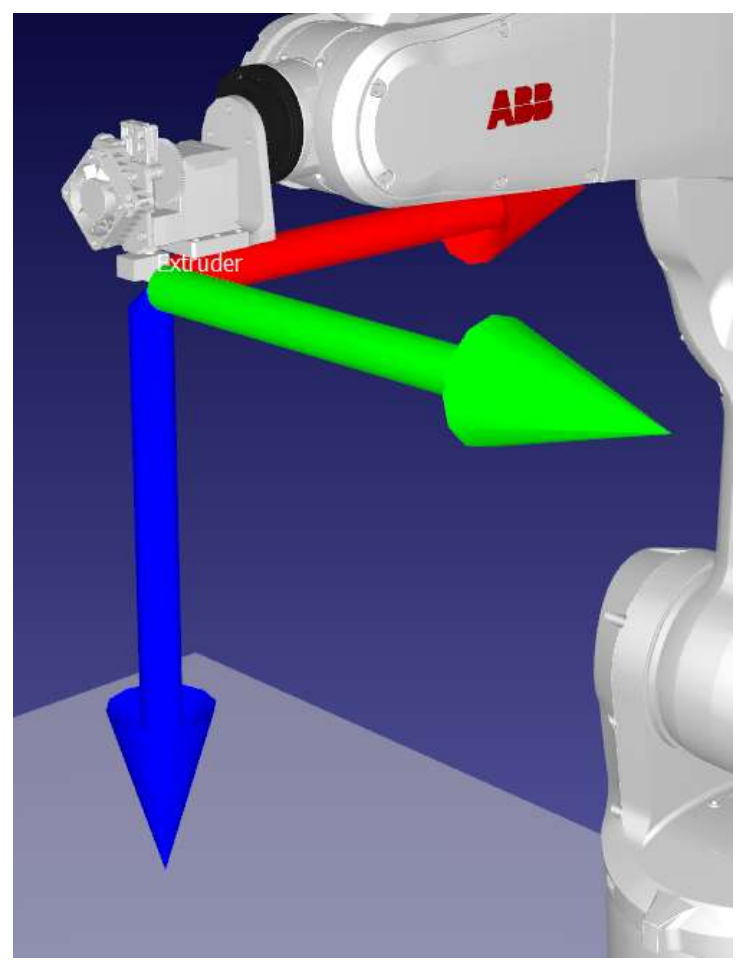

(A)

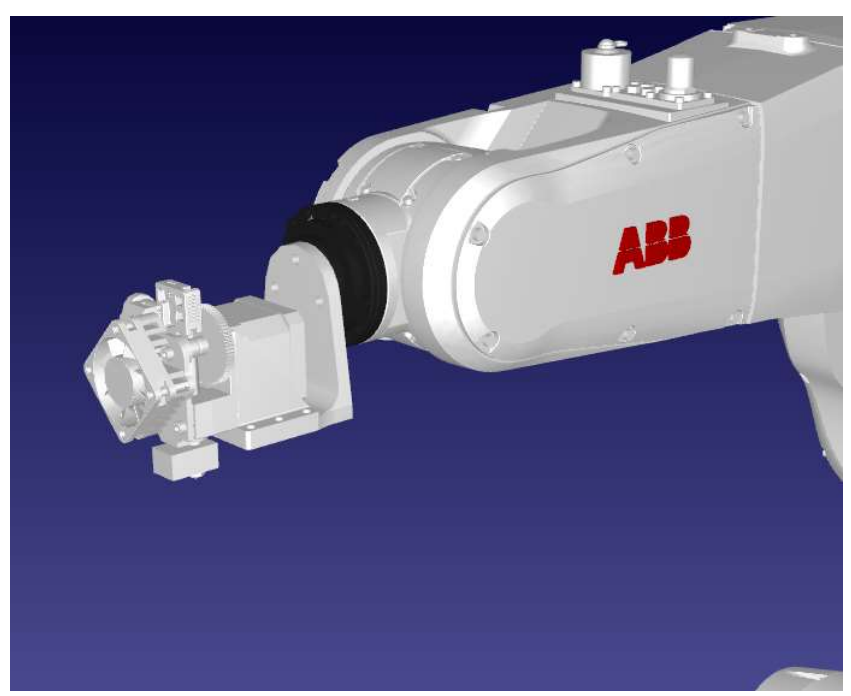

(B)

Figure 5.8 (A) TCP of Hot end visualised, (B) 3D model of Hotend assembly affixed to robot end effector

\subsection{Results}

Successfully printing using FFF was an entirely stochastic in balancing the many parameters for successful print. The most challenging parameter was with synchronizing the motion of the robot and the extruder head. As the stepper motor driving the extruder was not driven by the IRC5 controller, rather by its own PLC box, it was necessary to ensure that an appropriate amount of extrudate was deposited according to the distance traveled. To achieve 
this, several calibration cubes were printed while adjusting parameters to achieve acceptable results. The progress of calibrating the FFF parameters can be seen in Figure 5.9 which leads up to an ASTM D638 coupon and a twisted vase.

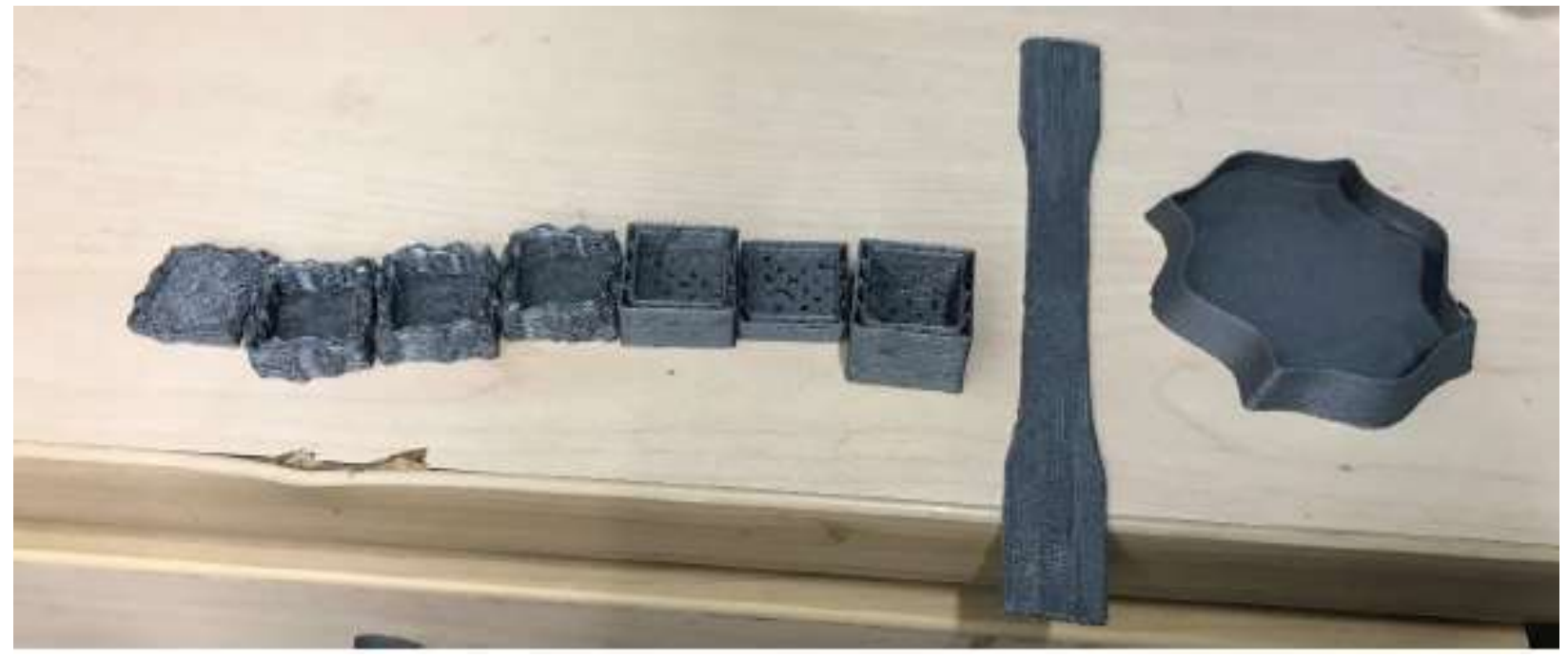

Figure 5.9 Progress of FFF parameters

A major issue that needed to be overcome for FFF to be feasible on the IRB1200 was the limitations on the program size and the speed and precision of the robot. The largest restriction was the file size limit. A single RAPID module is restricted in size to $8 \mathrm{MB}$, before it overloads the memory on the controller. An example of this issue can be seen in Figure 5.9, where the vase and the coupon had failed to complete due to the size of the file. For reference, the GCODE file for most parts may be several hundred megabytes in size. The limitation arises from the fact that that most Industrial robots preplan their trajectory after loading in the commands, rather than reading line by line on conventional 3D printers. The solution to this issue was to break up the program into many smaller programs and then dynamically load the program into memory. Essentially, as Part 1 of the program is running, Part 2 is being loaded. This continues until the final part of the program is loaded, after which the motion is terminated and the TCP is moved to a safe location off the part. 
To demonstrate the capability of the workcell, several test parts were printed to completion. The process can be seen in Figure 5.10, which shows the vase part in the middle of completion. The completed part can be seen in Figure 5.11. An ASTM D638 coupon was also printed to completion as seen in Figure 5.12. Additional details and a zoomed in view of the ASTM D638 coupon can be seen in Figure 5.13 to represent the quality that is achievable by the work cell once all the parameters are calibrated properly. 


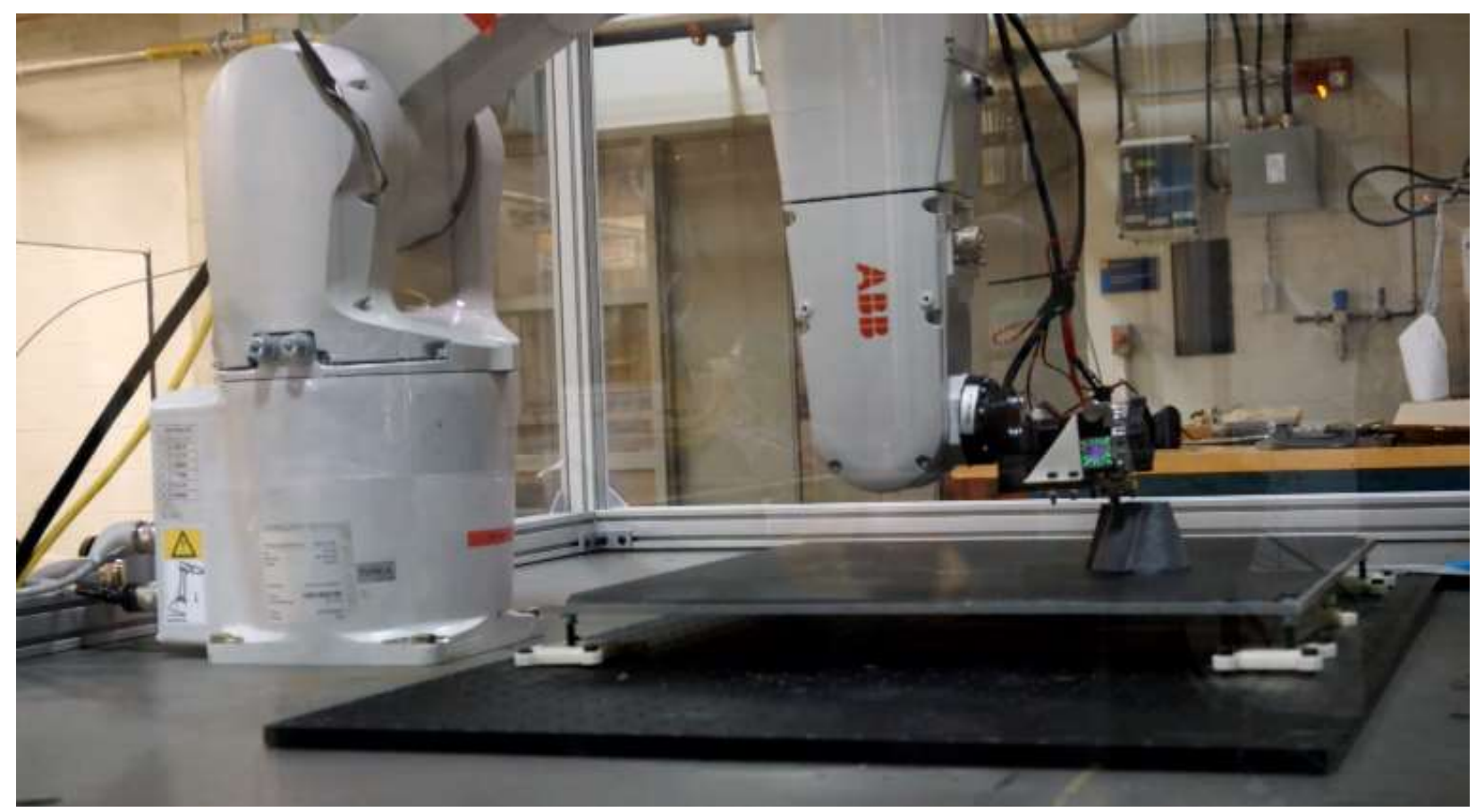

Figure 5.10 Picture of IRB1200 mid print

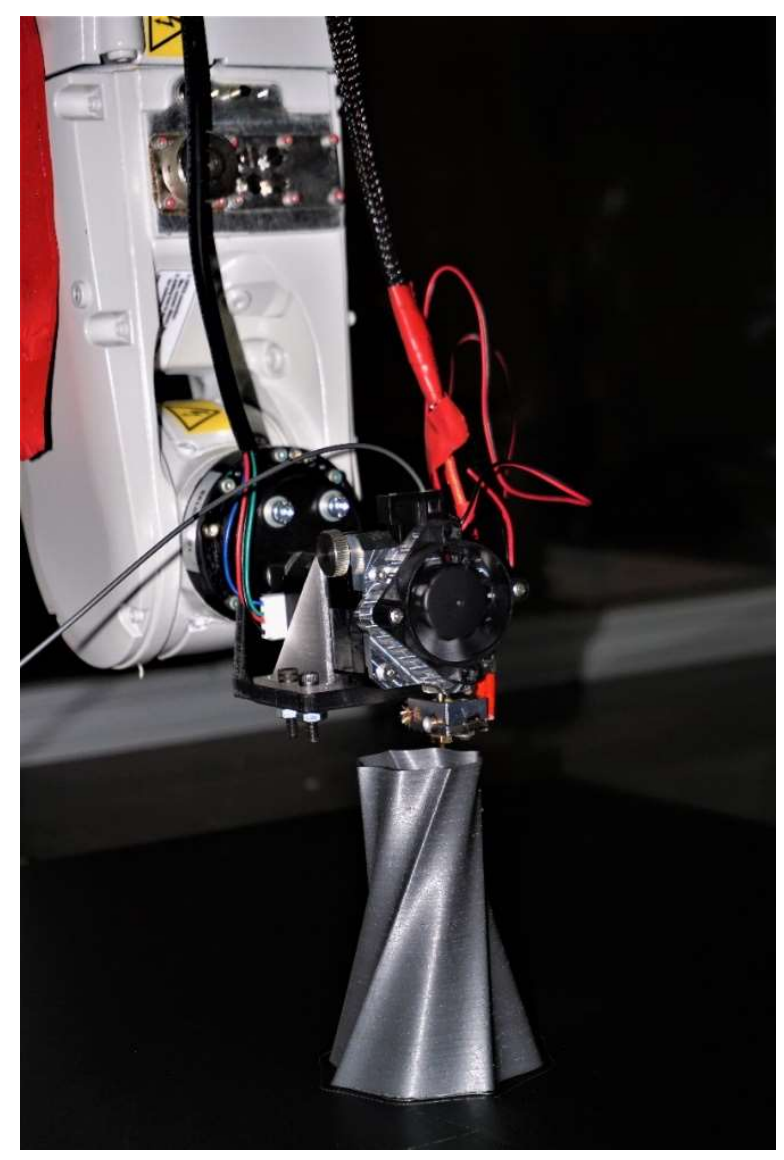

Figure 5.11 Vase Completed 


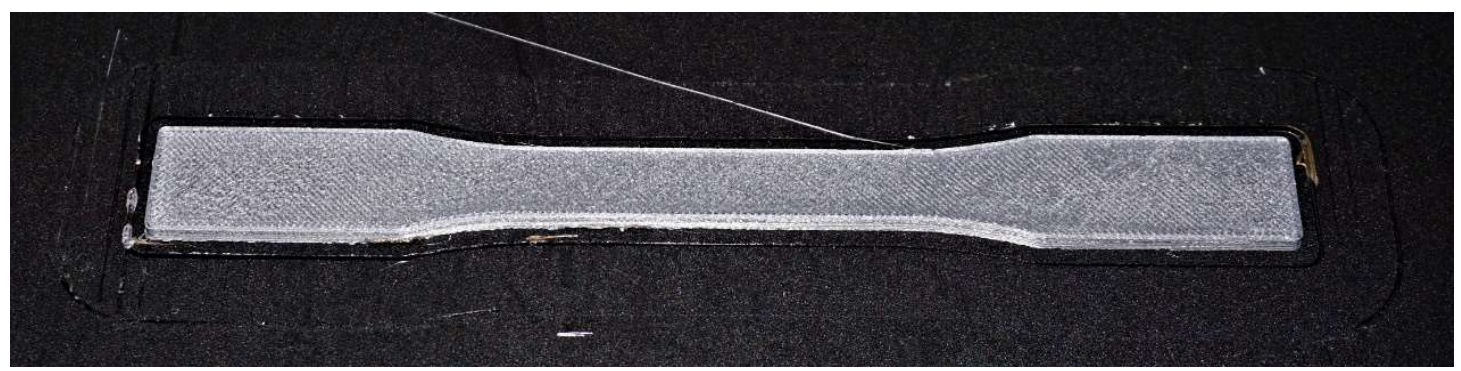

Figure 5.12 ASTM D638 Coupon Printed

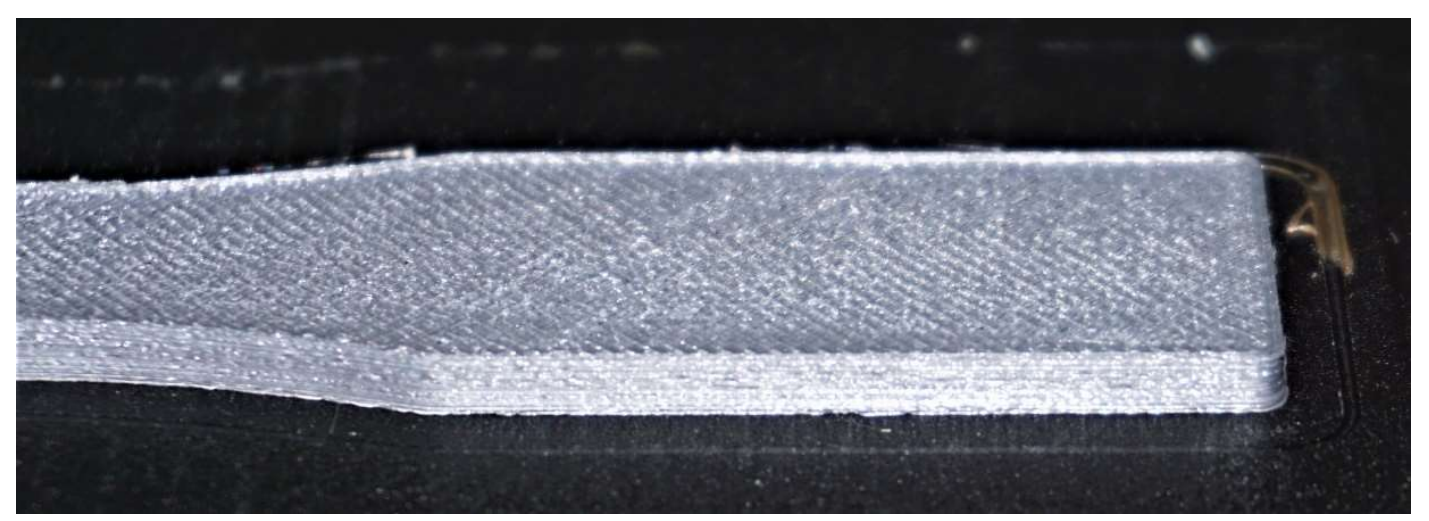

Figure 5.13 D638 Coupon zoomed in

\subsection{Conclusion}

3D printing using FFF is feasible and allows for greater degrees of freedom compared to a traditional gantry system. The design process for the FFF techniques using the IRB1200 allowed for easing the learning curve towards the Continuous Carbon Fiber deposition head. The learning curve reduction came namely in the programing of the robot, development of the heating systems, and the integration of tool heads onto the robot frame.

Future work is abundant, as only part of the total capability of the system was demonstrated. All the printed parts were still 2.5D prints. Multi-axis material deposition is a future consideration but will not be difficult to implement. The difficultly in multi-axis deposition remains in the tolerance of the tooling and the accuracy of the robot motion, as it will be 
necessary to guarantee that the material is always adhering to the surface of the tooling or the previous deposition. 


\section{CHAPTER 6 CONTINUOUS CARBON FIBER PRINTING HEAD DESIGN}

To validate CCVS, continuous carbon fibers would need to be steered at very small radii for the designs to be feasible. As previously mentioned in the literature review, 3D printing of continuous carbon fibers would be a viable strategy for manufacturing these highly steered parts. For that purpose, a tool head capable of depositing continuous carbon fibers could be required. As a commercial tool head was not available, it was concluded that a custom head had to be developed.

The tool head was designed with multiple purposes in mind rather than just validate CCVS. The previous section outlined the development of 3D printing via FFF on a robot workcell. The intention of that project was to allow for equipment that would be capable for multi-axis material deposition along curved surfaces like those commonly found in the Aerospace industry.

\subsection{Concepts Design}

The concept design was based on existing AFP and 3D printing technologies. The compaction system and cutting mechanisms were developed from concepts used in AFP and are not typically used for 3D printing purposes. From the 3D printing side, the fiber feeding mechanism and the heating method were used. As methodologies from both 3D printing and AFP were used, the technology can be considered a hybrid approach.

The basic schematic of the system can be seen in Figure 6.1 Configuration Ain the $A$ and B configurations. This is the base model from which the other configurations are derived. The following list describes the components labeled in figures Figure 6.1 to Figure 6.3.

1. Polished Nozzle \& Liner

2. Heating Block

3. Nozzle Carrier \& Heatsink 

4. Fan

5. Cutting Mechanism

6. Feed Tube

7. Feeding Wheel

8. Feeding Motor

9. Compaction Actuator

10. Compaction Wheel Carrier

11. Compaction wheel
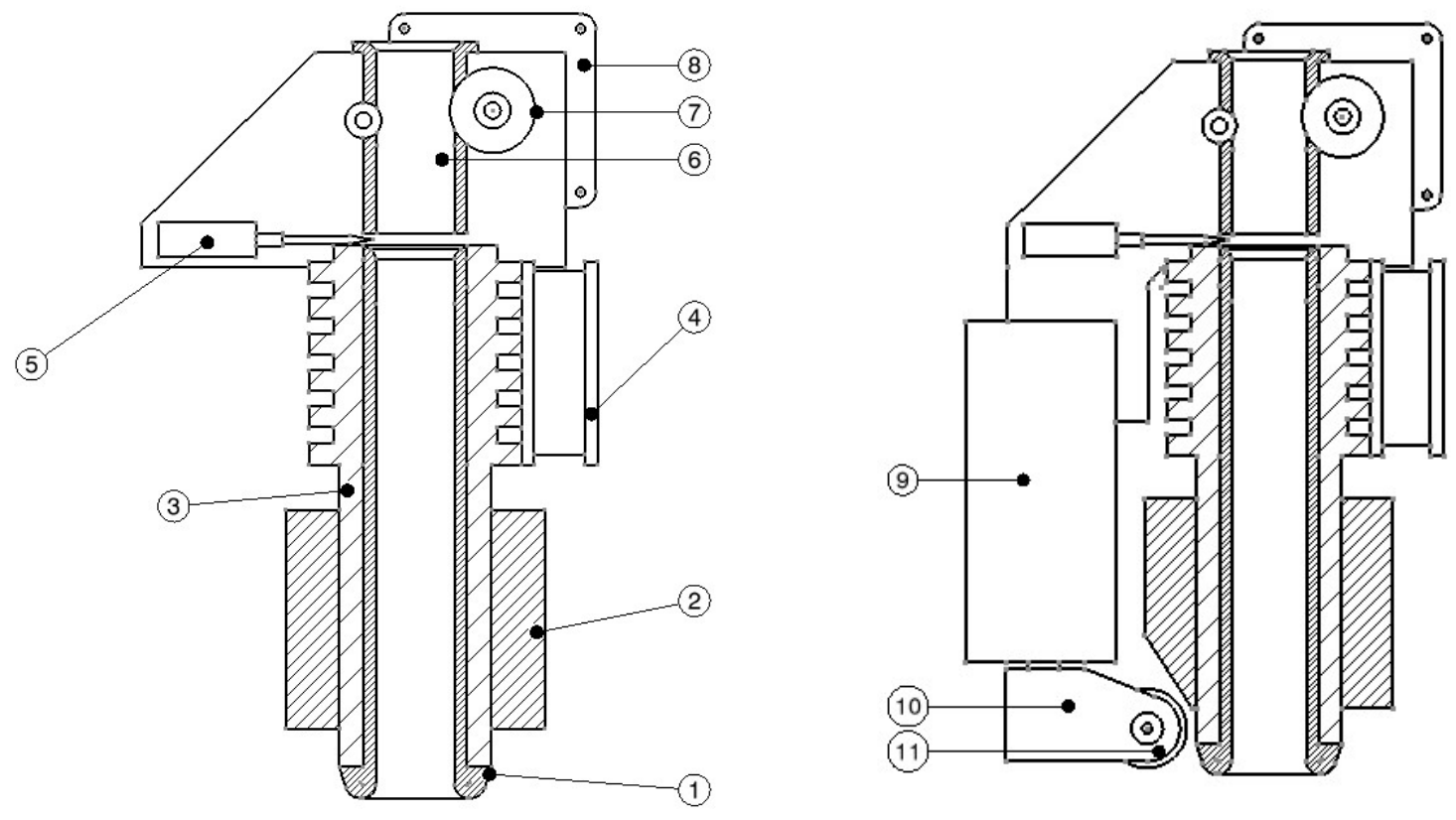

(A)

(B)

Figure 6.1 Configuration $A$ and $B$ 
The basic concept of operations begins with the fiber, which is initially fed into feed tube (6) using the feeding wheel (7) and motor (8). After the fiber passes through the nozzle (1), it is tacked on to the bed. At this point the motor disengages and the fiber is dragged on to the surface. In configuration A, the fiber is compacted using the nozzle head, which is flared to give relief against shear on the nozzle exit. As Tool Center Point (TCP) approaches the end of the fiber path, the cutting mechanism (5) actuates at a distance from the end of the path that is equal to the length of (1).

Additionally, if compaction is required, Configuration B offers a means of compaction using an Actuator (9). A silicone compaction wheel (11) is attached through a carrier (10) to the actuator. After the tacking of the filament, the compactor actuated to the desired compaction force using an integral load cell. The actuator deactivates at the end of the fiber path.

Configuration $\mathrm{C}$ is oriented with the compaction roller perpendicular to the build surface and the fiber extruder at an angle to aid in the initial tacking of the fiber. Configuration D is similar but with a parallel cut in the nozzle to aid in fiber feed and compaction. In both these configurations, the initial tacking is done with the compaction roller, rather than the nozzle as seen in the previous configurations. Configuration C and D are more typical in AFP tool heads. 


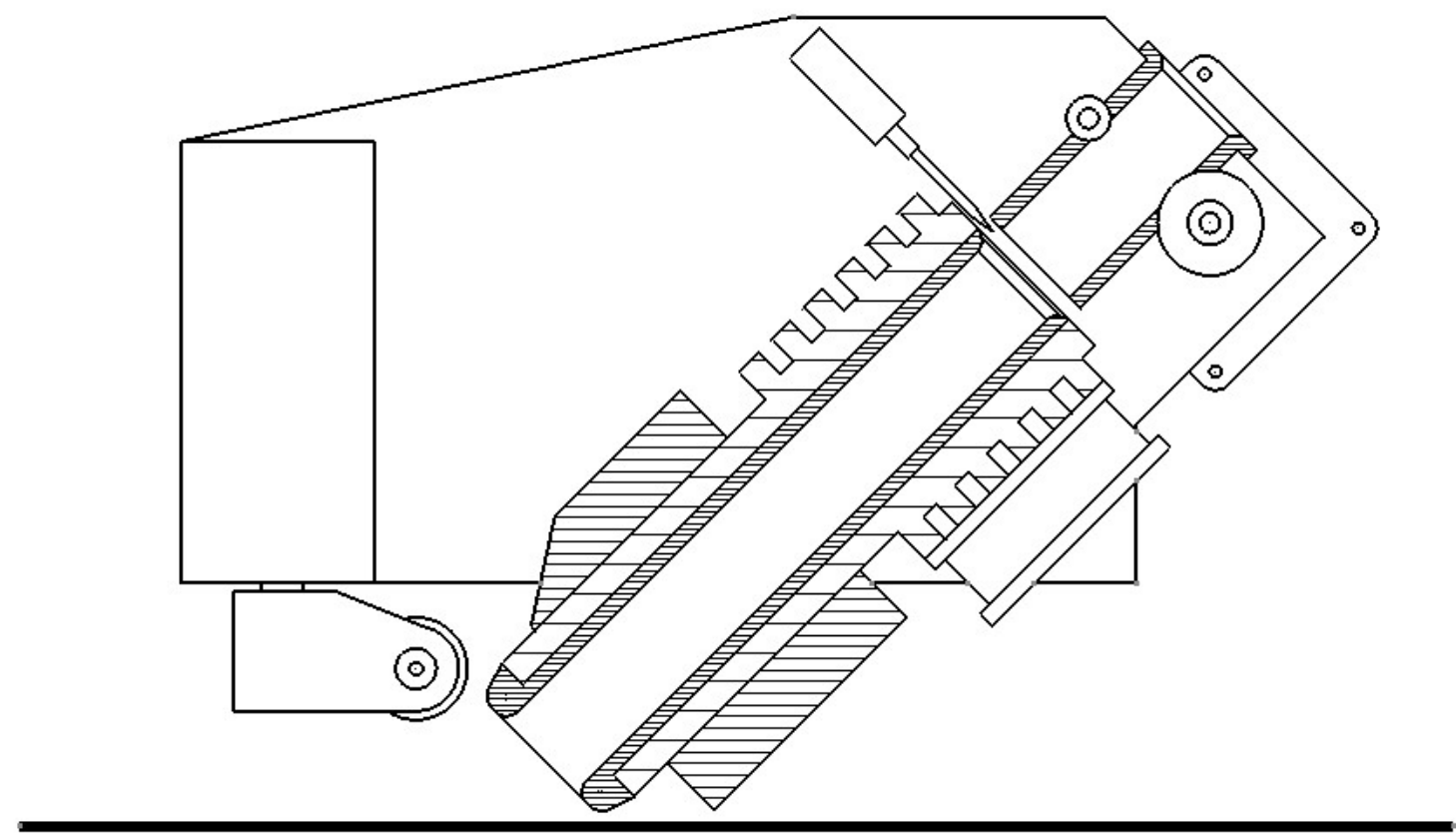

Figure 6.2 Configuration C

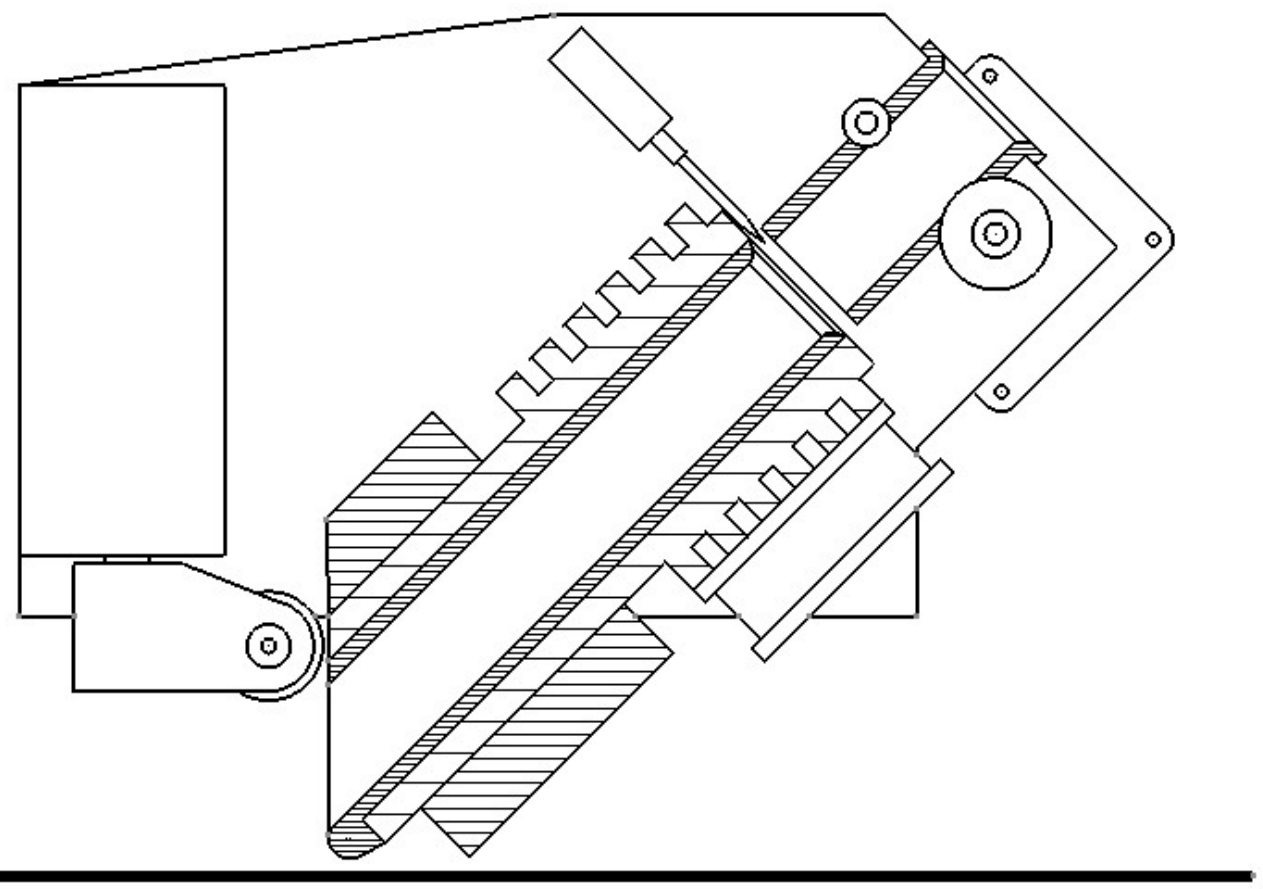

Figure 6.3 Configuration D 


\subsection{Prototype Development}

To validate the concept, an initial prototype with no feeding or cutting mechanism was developed. The fiber to be deposited was the Markforged Continuous Carbon Filament. The filament is an unknown carbon fiber impregnated with a proprietary Nylon resin. Details on the properties and composition of the prepreg are not published publicly by Markforged and make quality assurance difficult. To address this concern, Teijin Fibers, a prominent material supplier $\mathrm{t}$ has partnered with the FRAMES lab at Ryerson University for developing a prepreg material for the emerging additive manufacturing industry. A micrograph of the Markforged material provided by Teijin can be seen in Figure 6.4 and a zoomed in area in Figure 6.5. The material has a very high matrix content and poor wetting of the fibers resulting in voids.

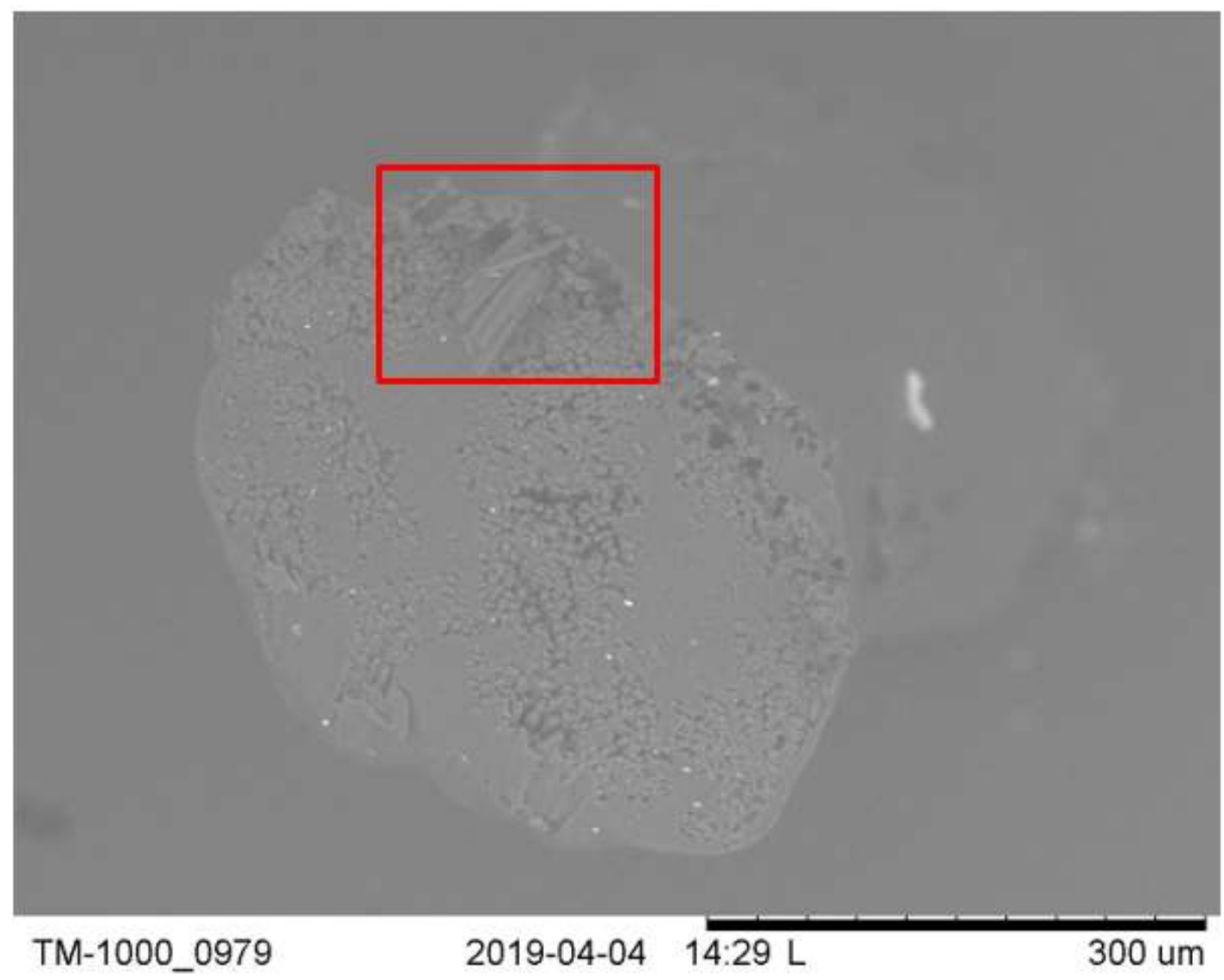

Figure 6.4 Micrograph of Markforged carbon fiber prepreg 


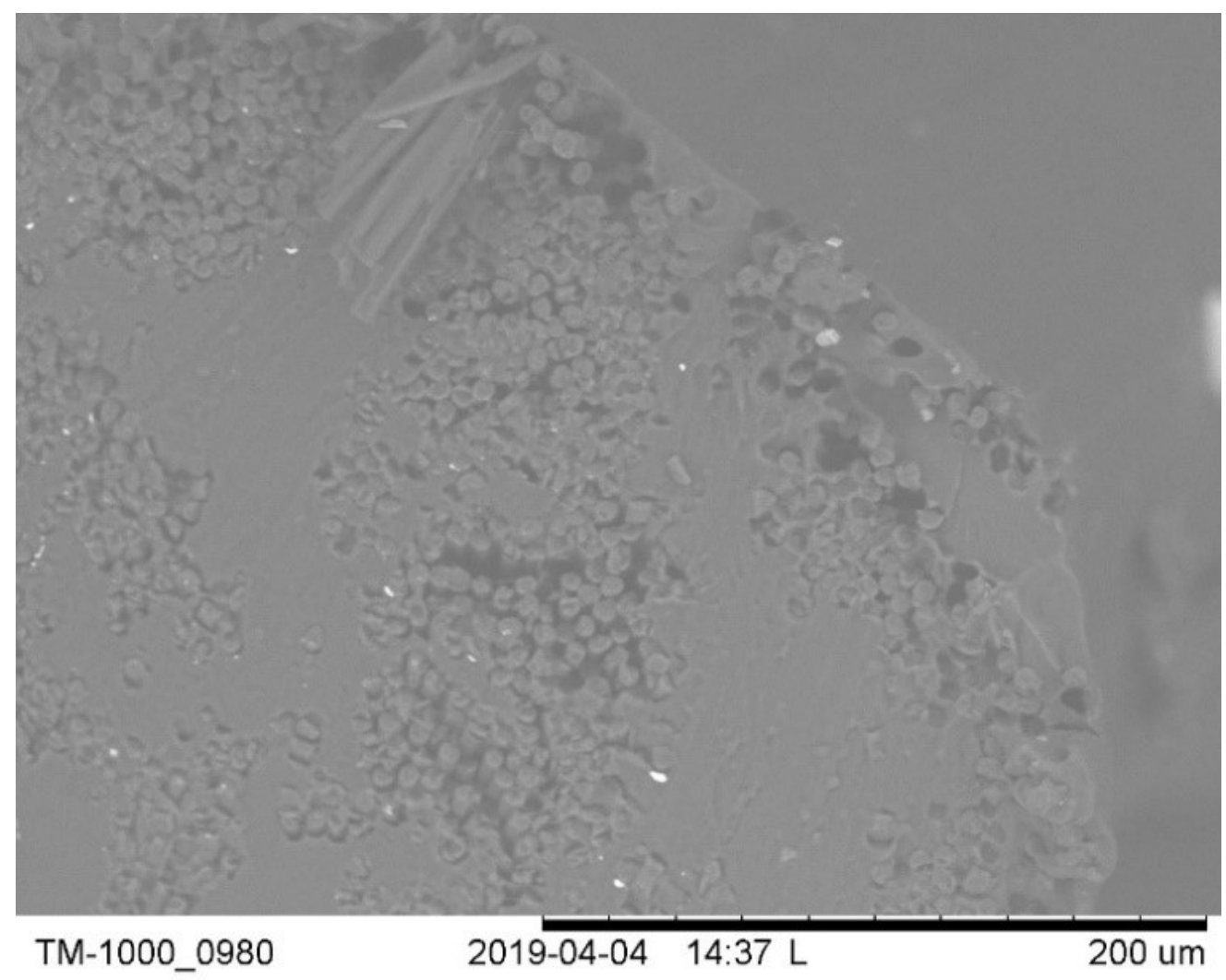

Figure 6.5 Zoomed in view of filament

A Markforged continuous carbon fiber nozzle was attached to an E3D V3 heat sink, which was fitted to the flange of the robot using an adapter that was 3D printed on a Zortrax M200 with their proprietary Z-Ultrat material. The prototype can be seen in Figure 6.6 affixed to the robot flange. 


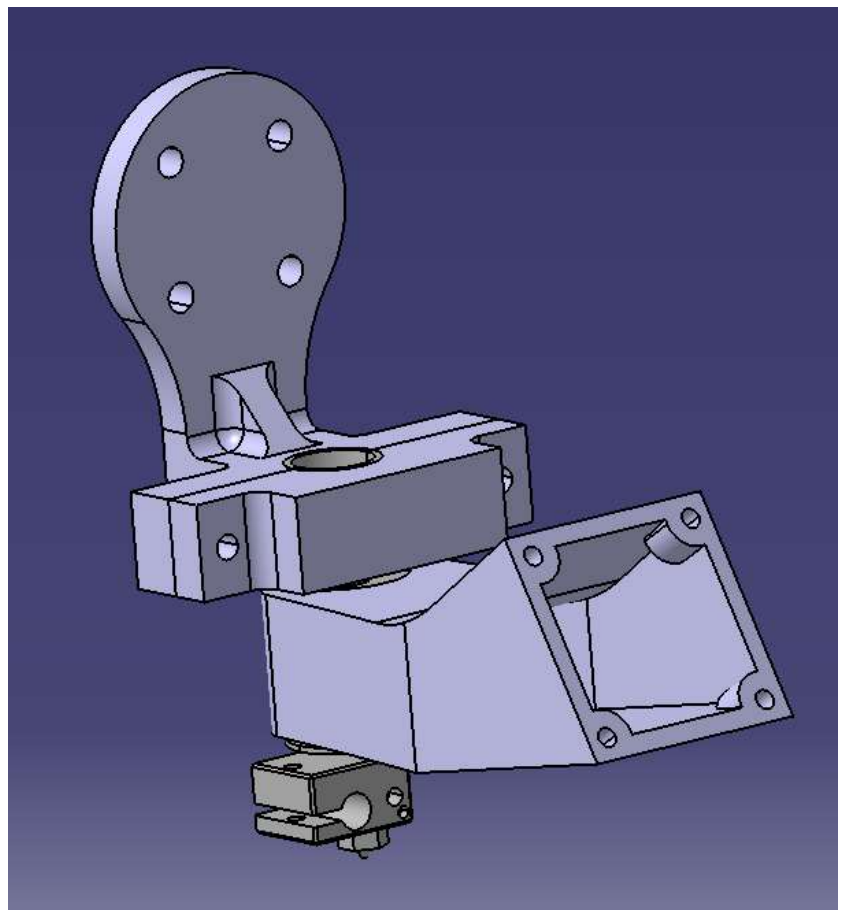

(A)

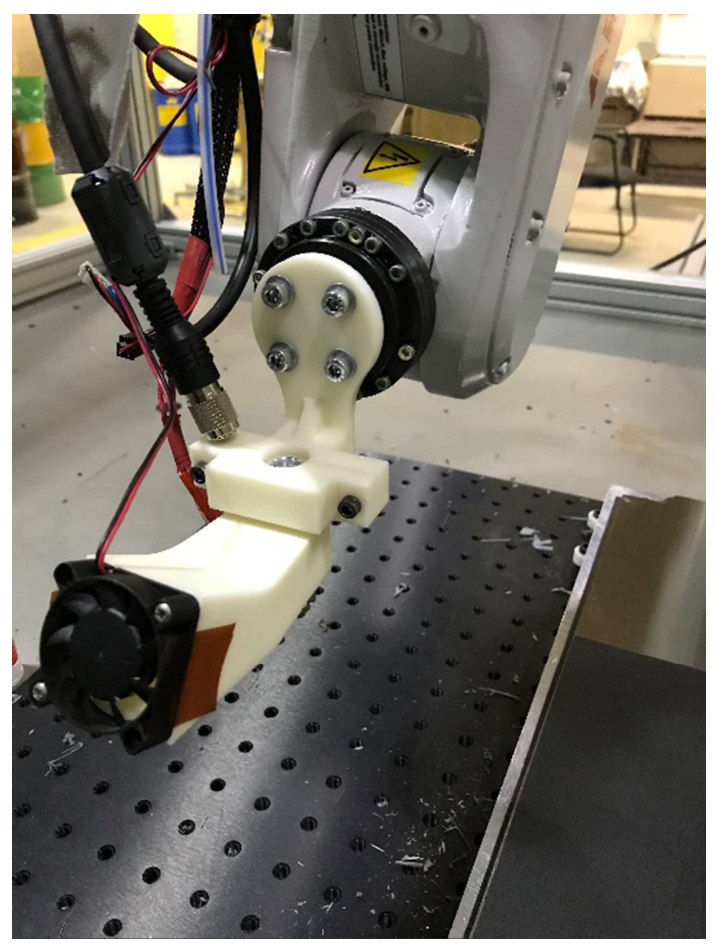

(B)

Figure 6.6 (A) CAD model of continuous carbon fiber hot end, (B) Integrated onto IRB1200

The bed was covered in Kapton tape, which is manufactured using a high temperature thermoplastic PEI. The PEI tape offers greater adhesion with the nylon matrix compared to a glass or aluminum surface.

For the purposes of determining the mechanical properties of the Markforged carbon fiber, a unidirectional ASTM D3039 coupon was manufactured. The suggested coupon is a straight rectangular coupon of $15 \mathrm{~mm}$ width and $250 \mathrm{~mm}$ length with a recommended minimum thickness of $1 \mathrm{~mm}$. Tabs were added to the coupons as suggested by the standard to prevent grip failures. The coupons were manufactured with a layer height of $0.1 \mathrm{~mm}$ resulting in 10 layers. The printing process can be seen in Figure 6.7. The coupons were to be tested to the ASTM D3039 standard, but ultimately were not, due to issues bonding strain gauges to the surface of the material and the slipping of the coupons on the grip tabs. The cause for these bonding issues were determined to arise from the Nylon resin itself and due to the surface roughness of the separately consolidated fibers. Nylon is commonly used as the container material for many adhesives which therefore reduced the effectiveness of bonds with the strain gauge adhesive. 
The surface roughness was significant enough that the adhesive was not enough to fill the voids. The roughness is assumed to have been created due to the poor material impregnation of the prepreg. Nevertheless, the concept was proven and it was chosen to move on to the final design.

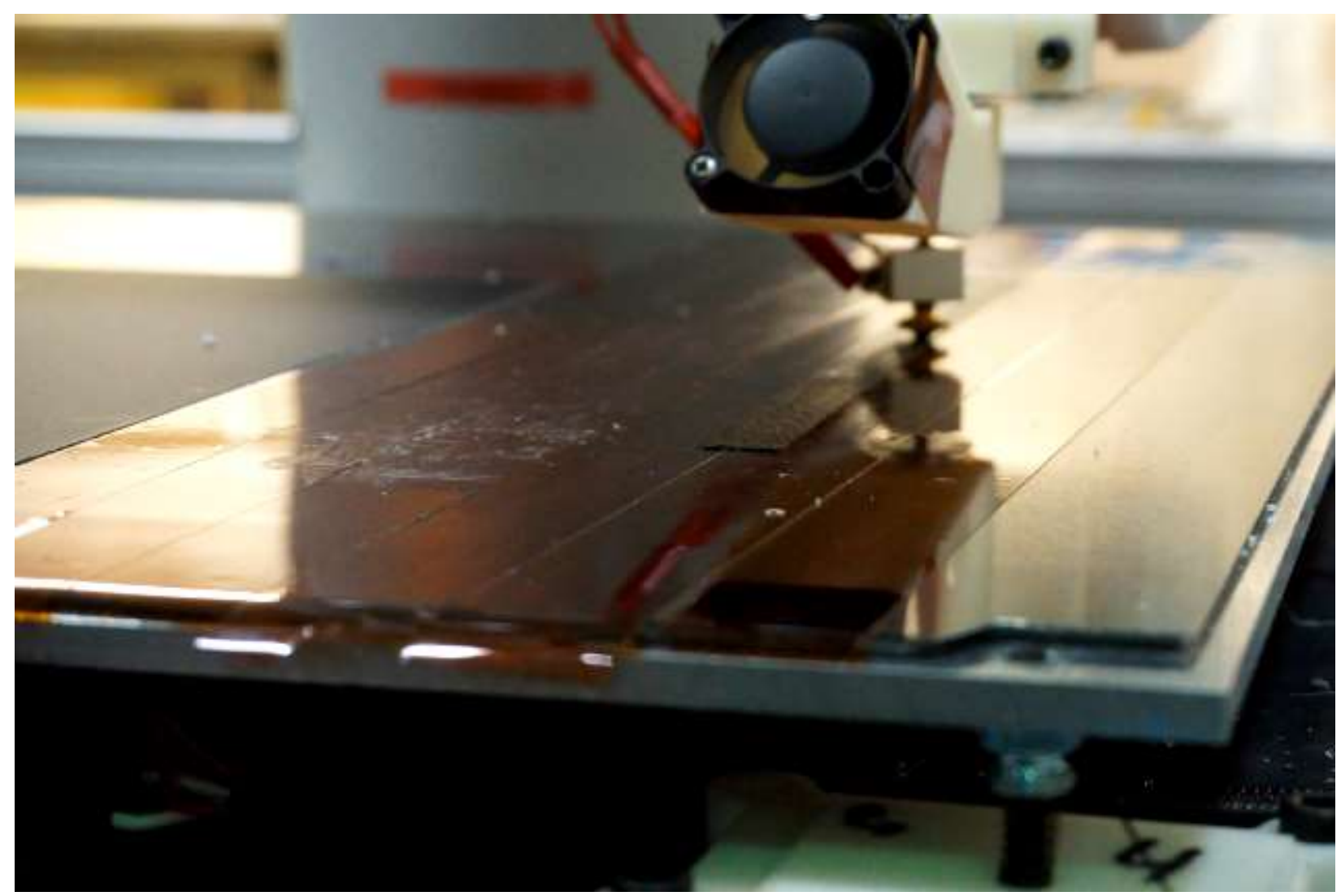

Figure 6.7 Continuous Carbon Fiber printing

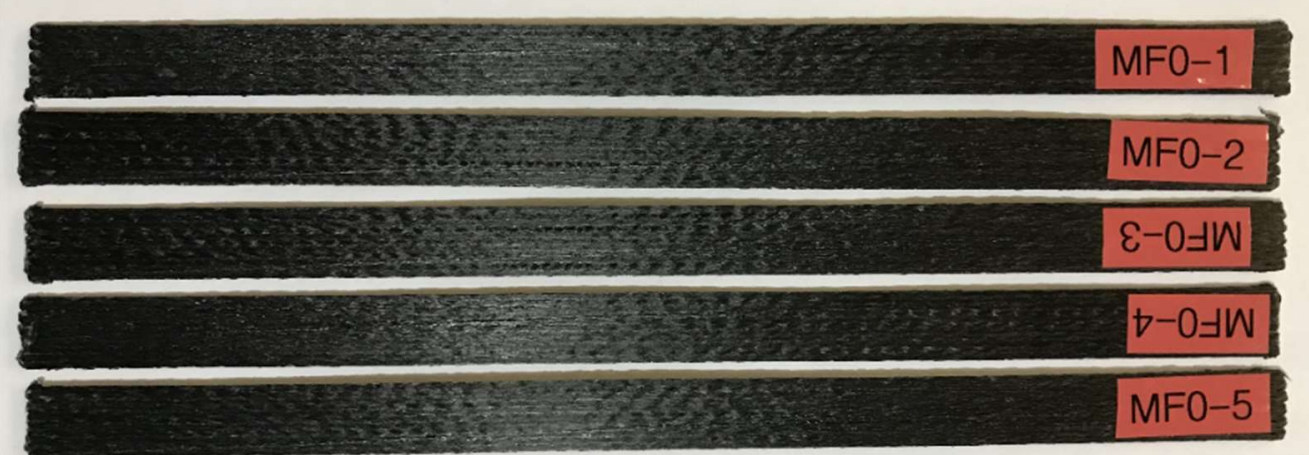

Figure 6.8 ASTM D3039 Tensile test coupons manufactured with Markforged Continuous Carbon Fiber 


\subsection{Continuous Carbon Fiber Head Design}

After overcoming the learning curve of printing with the Markforged continuous carbon fiber filament, it became feasible to transfer the methodology to a more robust and purpose designed tool head. From the prototype design, it became evident that a compaction system was required for tacking the filament onto the tool surface or the previous layer. Compaction was achievable from the nozzle design itself. Therefore a combination of the previously mentioned Configuration A and B was designed.

The full CAD model can be seen in Figure 6.9. A physical prototype was manufactured from this design. The following list describes the components labeled in figures Figure 6.9 to Figure 6.10.

1. ATI Robotic Tool Changer

2. Carriage Assembly

3. Linear Actuator

4. Hot End Assembly

5. Stepper Motor

6. Servo motor

7. Heater cartridge and nozzle

8. Cooling Fan

9. Heatsink

10. Extruder Housing and tensioning system

11. Fiber feed Quick connect fitting

12. Cutting mechanism

The CFF heat consists of two main assemblies: The Carriage Assembly (1) and the Hot End assembly (2). The assemblies are connected using a linear actuator (3). The super assembly is affixed to the robot interface with a tool-changer (1). The fiber is fed through tube attached to the quick connect fitting (11). The fiber is fed through the tensioning mechanism (10) which is attached to a stepper motor (5). The stepper motor feeds the fiber through the cutting mechanism (12). At a prescribed distance, the cutting mechanism will actuate and release the 
fiber from the rest of the spool. The fiber continues through the heat block and nozzle (7) onto the part.

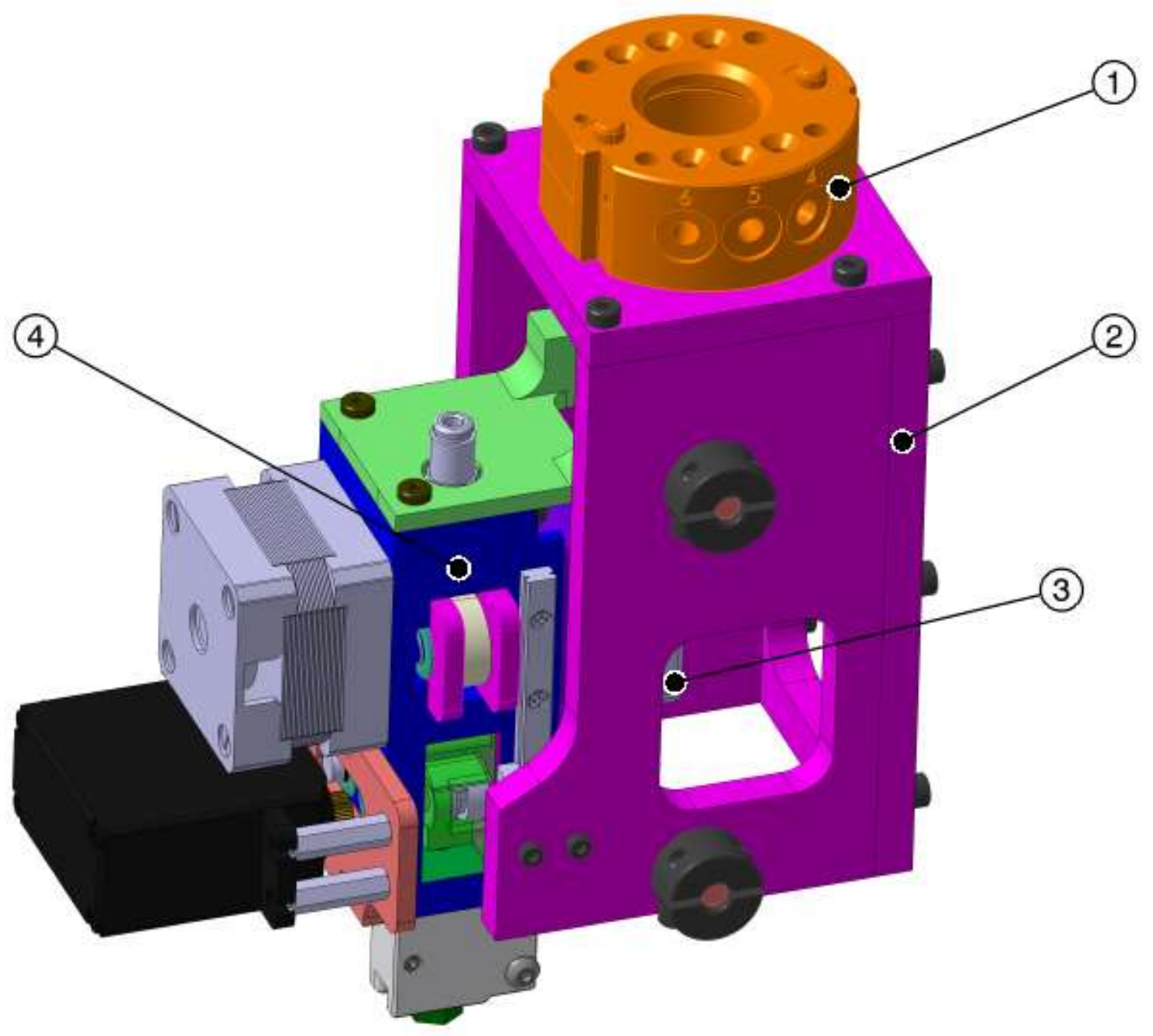

Figure 6.9 Full assembly of the printing head 


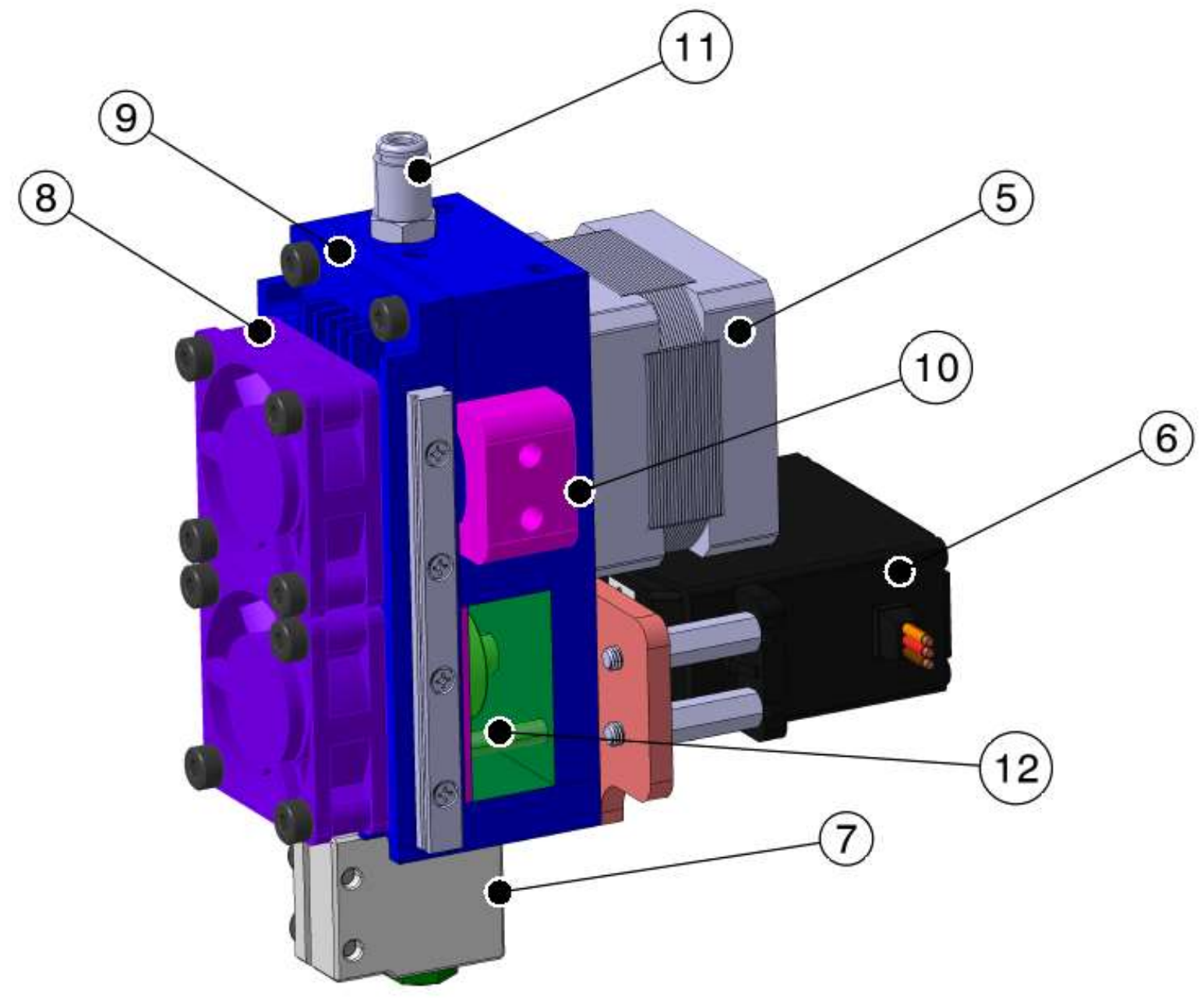

Figure 6.10 Hot end assembly

The assembly is mostly constructed from machine 6061-T6 with the exception of the heat sink and the nozzle. The heat sink was manufactured from a copper alloy for better heat conductivity. 


\subsection{Nozzle Design}

A specialised nozzle needed to be developed for the purposes of printing continuous carbon fiber/PEEK. For the purposes of carbon fiber deposition, the matrix is molten and then pressed on the previous layer or the build plate with a flat portion.

The nozzle was manufactured from 316 electroless nickel plated stainless steel. The stainless steel was chosen for adequate stiffness, as the nozzle also doubles as the compaction surface and for dimensional stability when it is heated up to the temperatures required to melt PEEK. The nickel platting was required to provide a lubrication surface for the PEEK to flow freely without sticking and for erosion resistance against the carbon fiber rubbing against the internal surfaces during deposition.

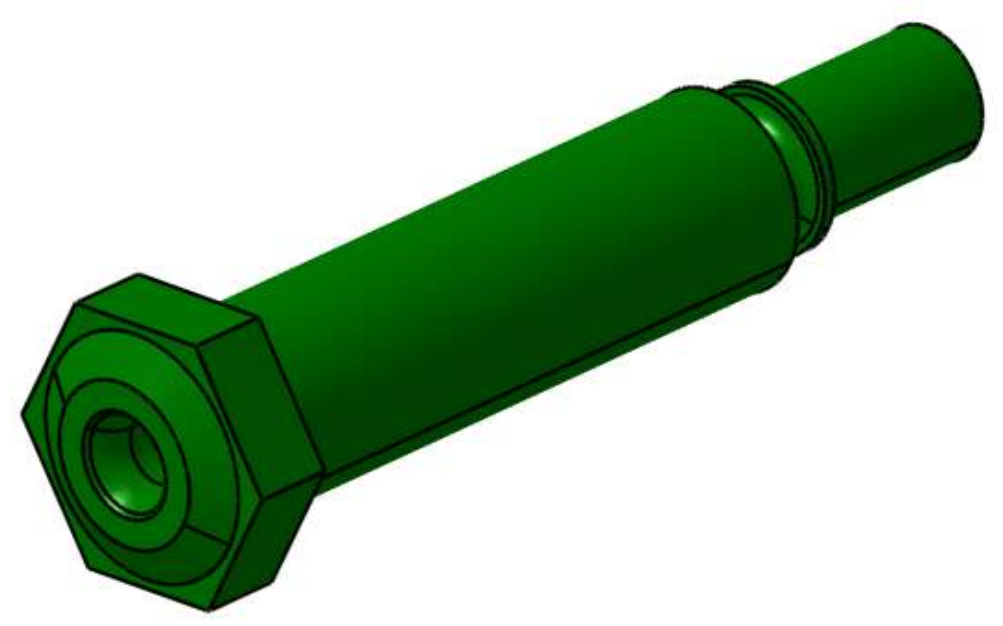

Figure 6.11 Nozzle Design

The nozzle exit was designed to be flared to prevent the fiber from breaking when deposited into the part. The angle of the flare was set to 45 degrees to allow for a gentle slope on to the part. During deposition, the fiber will be rubbing up against the flared surface, which is 
why the previously mentioned electroless nickel plating was required. Furthermore, the end of the nozzle was a smooth flat surface to aid in the compaction of the deposited fiber.

\subsection{Cutting Mechanism}

A drum cutting mechanism was developed as a compact solution compared to a linear shear cutter. The drum is a revolute body with a centered slot for the fiber to pass through. A urethane wheel provides the backing surface for preventing the fiber from slipping. For cutting the fiber, a servomechanism attached to the drum cutter actuates and a blade passes over the filament to contact the urethane wheel, thereby breaking the filament. After the cut, the blade returns to its original position.

\subsection{Extruder Mechanism}

A tension style extruder was designed for feeding the filament into the hotend. The design presses two urethane wheels together using spring tension that can be adjusted. A stepper motor mounted to one of the wheels then drives the fiber forward. Furthermore, an encoder is attached

to the feeding mechanism to determine the amount of filament that has been deposited to confirm 


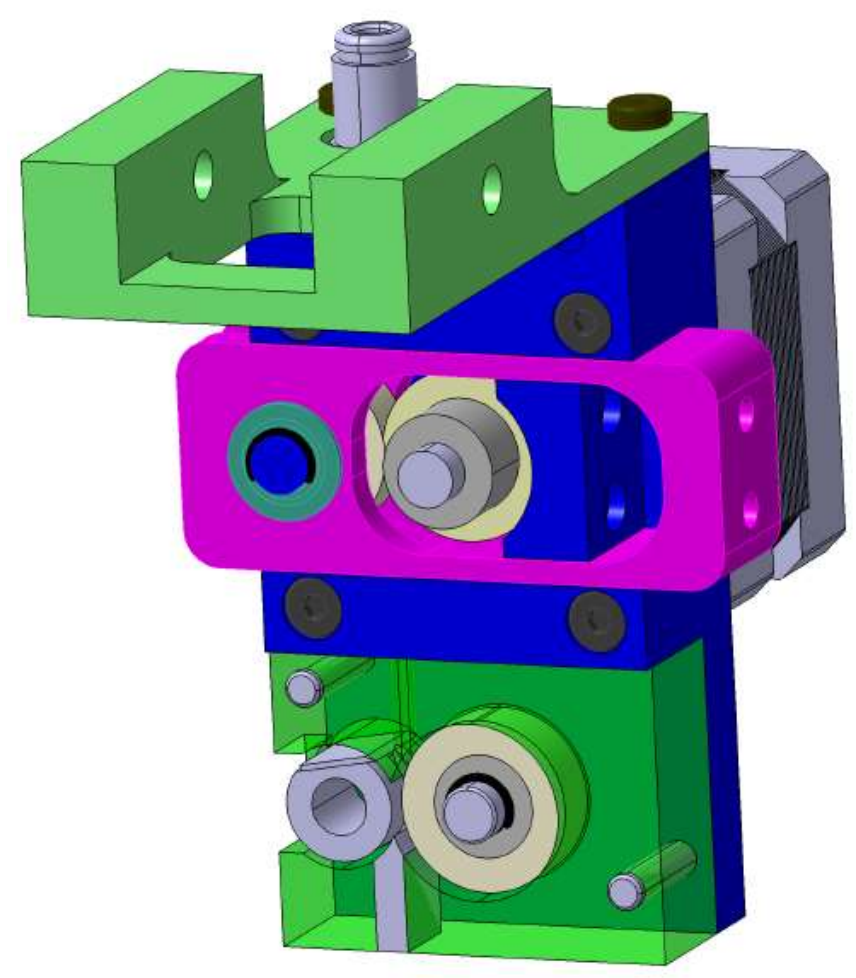

Figure 6.12 Tensioning and Cutting mechanism

\subsection{Conclusion}

A continuous carbon fiber 3D printing head was designed and prototyped to prove the feasibility of using an industrial robot to print continuous carbon fiber. A non-cutting prototype was used to create test coupons according to the ASTM D3039 standard successfully from which a final design was generated. The final design is currently under assembly as of the publication date of this thesis and will be subsequently used for future test coupons and designs.

The development of the tool head is a challenging process with many question pertaining to the performance and functionality of the tool head still remaining to be determined. A major concern is the fiber cutting mechanism, which is one of the two systems that have not been thoroughly prototyped and tested, the other being the compaction system. Jamming of the fiber after arriving at the minimum cut length or due to fiber buckling remains a concern and will 
require considerable testing to achieve a reliable design. The compaction actuator is a lesser concern due to the simplicity of its motion and function, but a heuristic approach will be required to solve the initial tacking of the fiber, which is aided by a number of "rules of thumb" developed over time in the AFP discipline.

Future work pertains to improving the deposition rate of the fiber and quantify the properties of parts manufactured from the tool head. Furthermore, there will need to be additional components and subsystems added to the tool head for quality assurance. A key example would be a load cell system to maintain a constant compaction force and a deformable compaction roller to prevent crimped fibers when laying up on complex surfaces. 


\section{CHAPTER 7 IN PROCESS INSPECTION}

An inspection process is necessary to ensure the quality of the part that is being manufactured. Most techniques for inspection are post process techniques, which are only capable of detecting defects after the part is completed. In additive techniques, it is preferable to be able to catch defects as they are produced to either reject or repair a part. An in-process inspection or in-situ inspection procedure is described in this chapter to address the needs for a true quantitative in-process inspection system.

A prototype system was developed using a LJV-7080 laser line scanner from KEYENCE that was integrated on to an ABB IRB1200 industrial robot. The laser scanner uses a laser triangulation technique to project a laser line across the surface to read the height data across that line. For the purposes of proving the concept, the LJ-V7080 system was integrated onto a E3D FFF hot end. The FFF process was interrupted after completion of each layer to take a scan of the layer. The scan data was post processed in MATLAB to render the images of each layer. The in-situ defect detection and repair are a future work and not considered in this thesis outside of a theoretical basis.

\subsection{KEYENCE LJ-V7080}

The KEYECE L-V7080 was selected after considering several other inspection techniques and offerings from other companies. As seen in Figure 7.1, the LJ-V7080 provided the best resolution and was able to clearly capture the topology of the test coupon. As an alternative to the laser line scanner, a 3D handheld light scanner from Creaform was tested but the results were not satisfactory. Although the software support and quick stitching of the 3D surface using the Creaform scanner was beneficial, the resolution was not as high as the other two scanners as it was intended to scan large objects. 


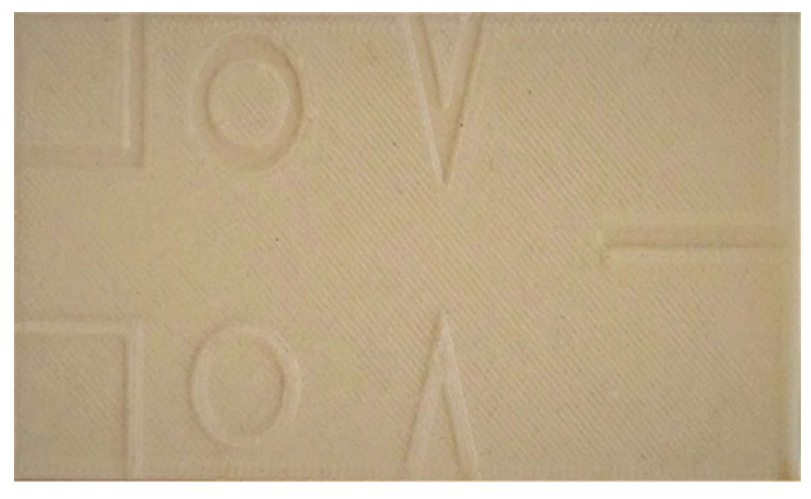

(A)

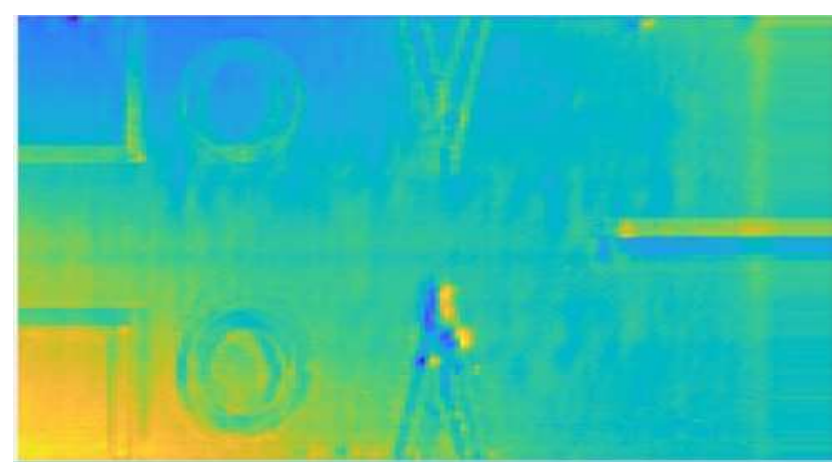

(C)

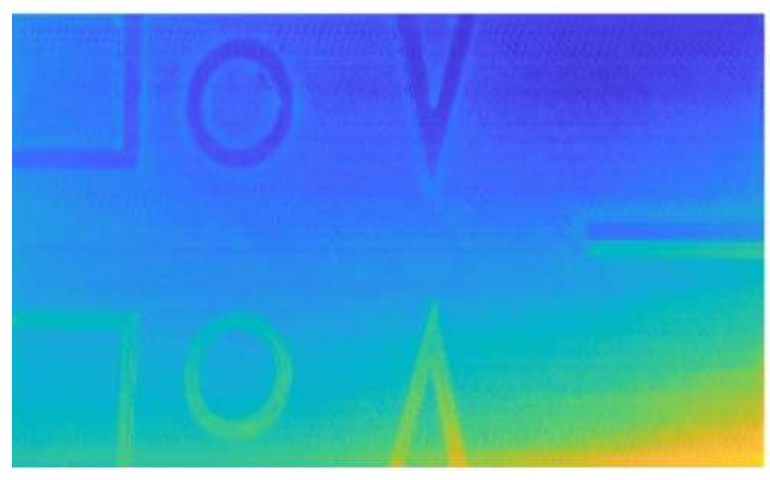

(B)

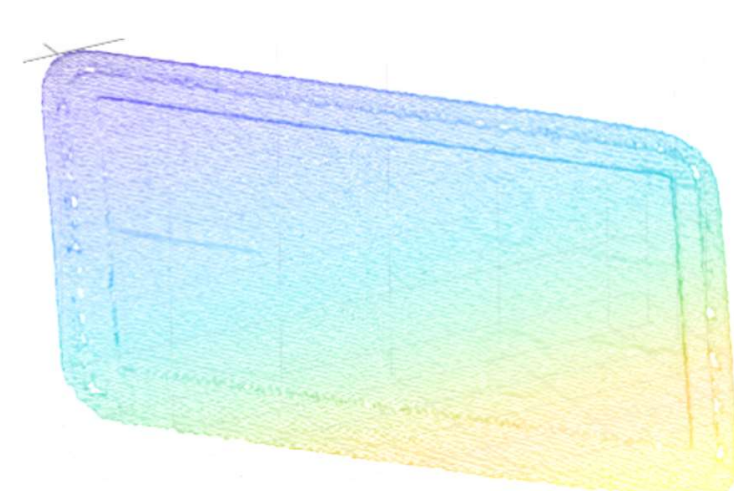

(D)

Figure 7.1 (A) Reference 3D printed Part, (B) Keyence LJ-V7080,(C) Micro Epsilon, (D) Creaform

The system consists of two components, the laser head its self and the controller box. The laser head is compact enough to fit next to a E3D hot end. A schematic of the head can be seen in Figure 7.2. The width of the measurement area is in the range of $25-35 \mathrm{~mm}$ with a sample of 800 individual height measurements. Therefore, the resolution of the scan is proportional to the distance of the laser above the part surface. 

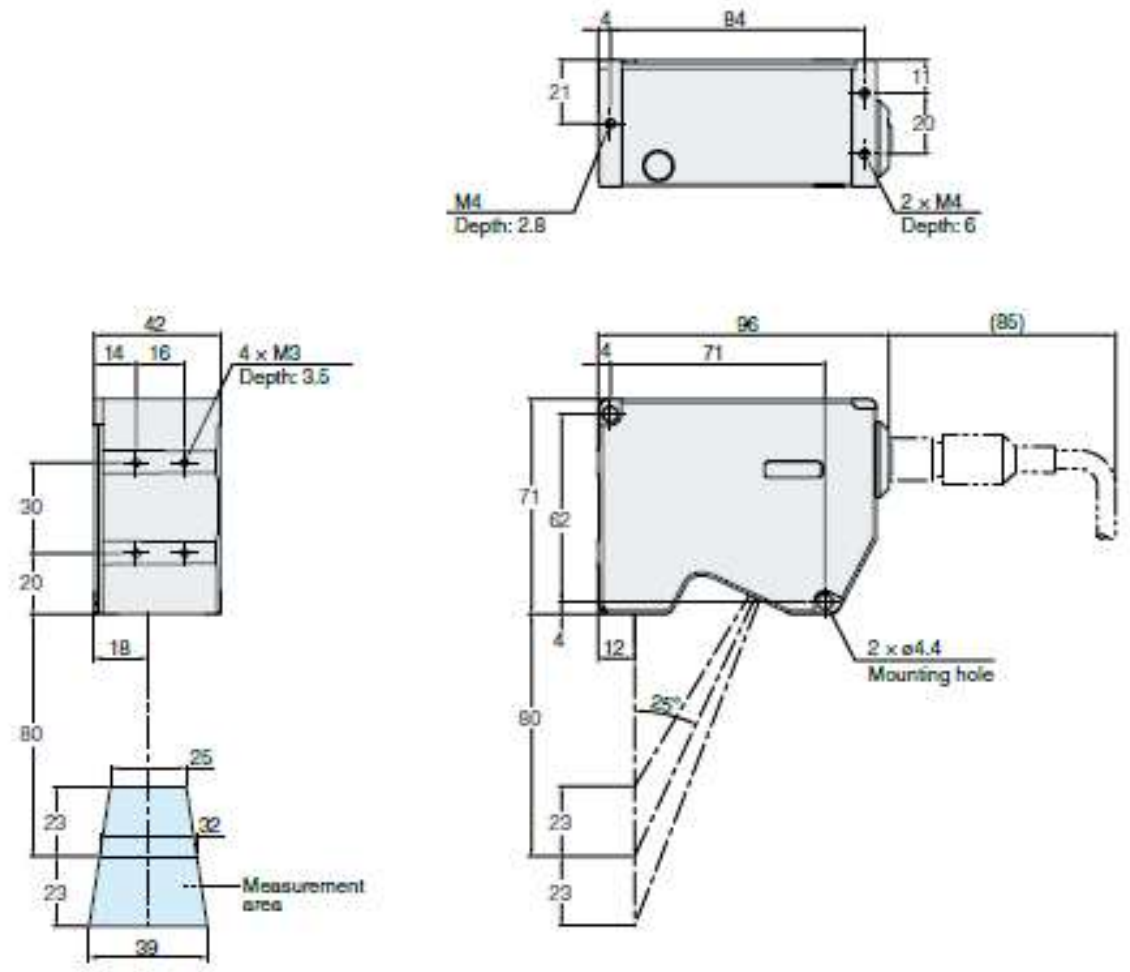

Figure 7.2 Technical information regarding LJ-V7080 [63] 


\subsection{High level Procedure}

An ASTM D638 Type I coupon was chosen as the article for inspection. The rational for this choice was that the coupon width fits within the measurement range for the L-V7080 and thefore a stiching algorithm would not be required. Furthermore the D638 coupon is a standard test article and therefore any results that were obtained from the scan could be correlated against standardised tests. The coupon that was scanned consisted of 24 layers with a layer thickness of $0.14 \mathrm{~mm}$. The stackup sequence considered was [90 - 45450$]_{3 s}$ with two shell outlines. The material used was Prusa Research PLA 1.75 mm filament.

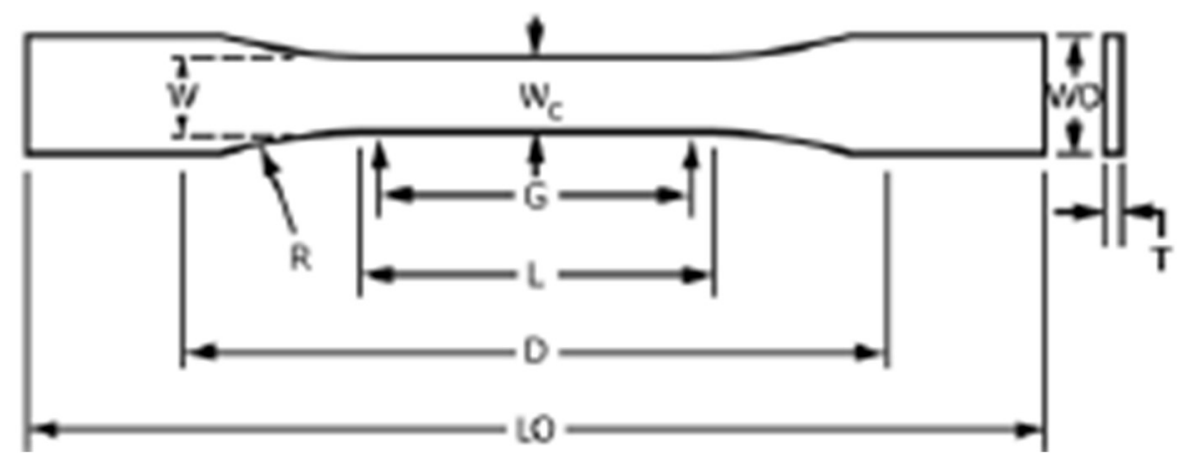

Figure 7.3 ASTM D638 Coupon dimensions [64]

The process is outlined in a flow chart in Figure 7.4. The procedure begins with the slicing of the CAD data of the D638 coupon and generating the GCODE for the print. Any slicer may be used as long it as it is able to output g-code and the settings used reflect the hardware of the extruder. Using ROBODK, the GCODE output from the slicer is post-processed into the RAPID code with the corresponding FK-IK solver for the IRB1200. The post-processor for ROBODK was modified to include an interuppt between layers so that a scanning subroutine is called. When the IRC5 controller enters the scanning subroutine, the TCP is changed to the LJ-V7080 and is moved along the centre of the coupon from end to end. The results of the scan are saved to a CSV file on the desktop computer. At this point the coupon scan would be post processed and the results compared to a acceptable baseline after which a decision to reject or repair the part 
would be made. The comparison procedure was ommited in this project due to complexity but will be implemented in future iterations.

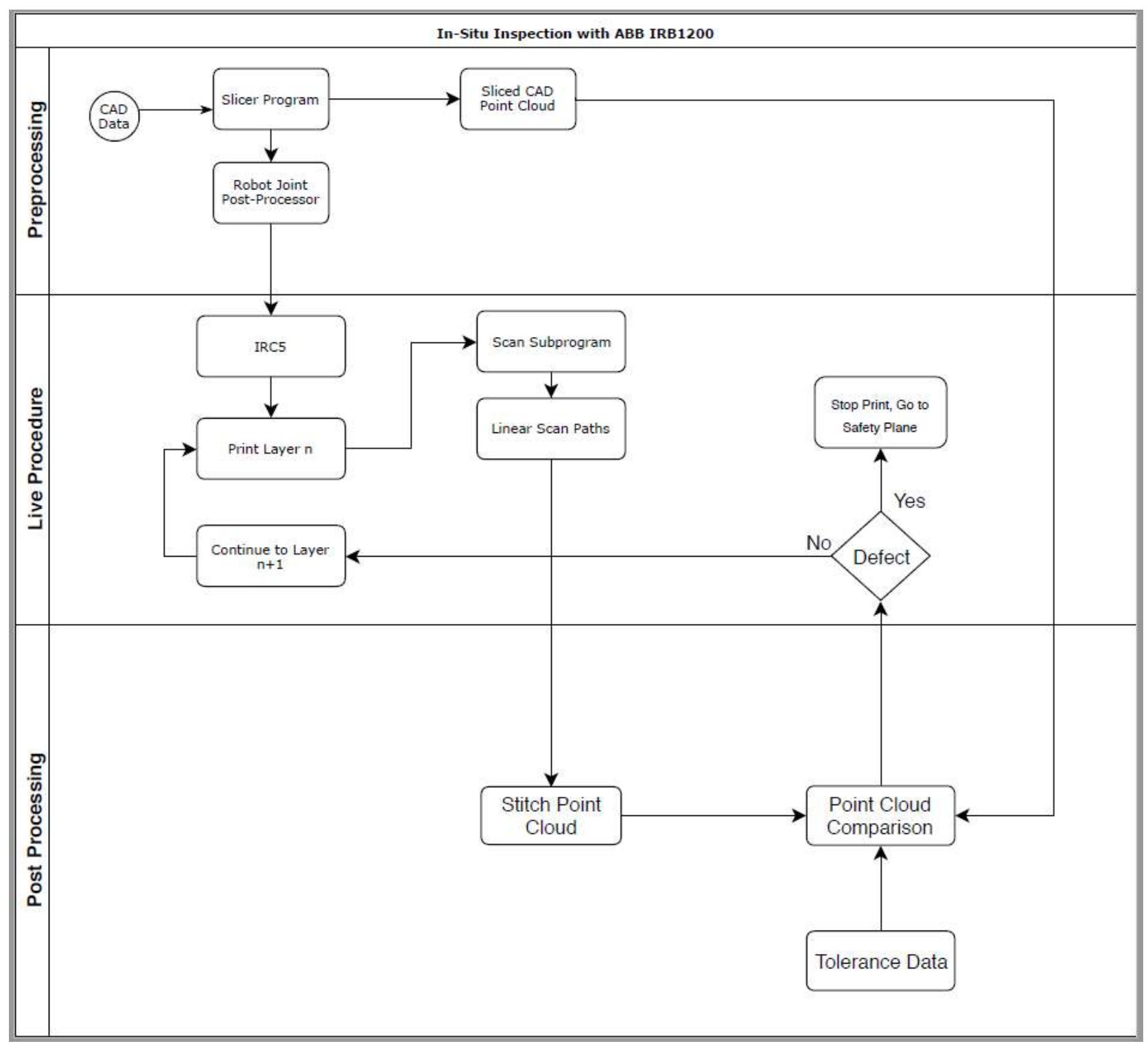

Figure 7.4 Inspection Process flow chart 


\subsection{Laser Hardware Integration with ABB IRB1200}

The existing E3D Titan Aero hot end was modified to accept the LJ-V7080 laser scanner, which was to be directly fitted to the IRB1200's end effector interface. The positioning of the laser was deliberate to avoid heat from the nozzle, which was offset to the left side of the hotend. As the laser had a maximum allowable temperature of $60^{\circ} \mathrm{C}$, it was beneficial to mitigate as much heat as possible. The mount was manufactured using a FFF process through a Zortrax M200 with the proprietary Z-Ultrat material.

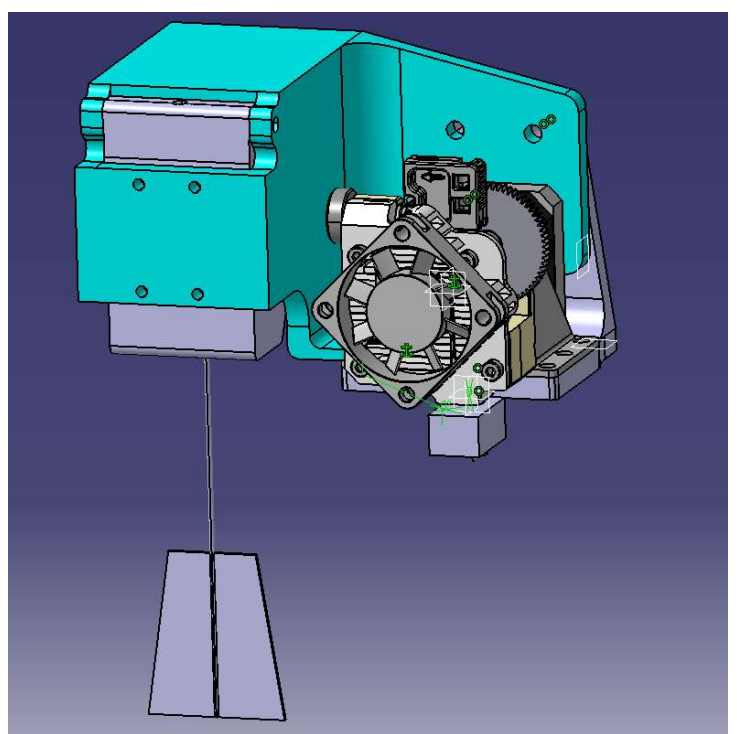

(A)

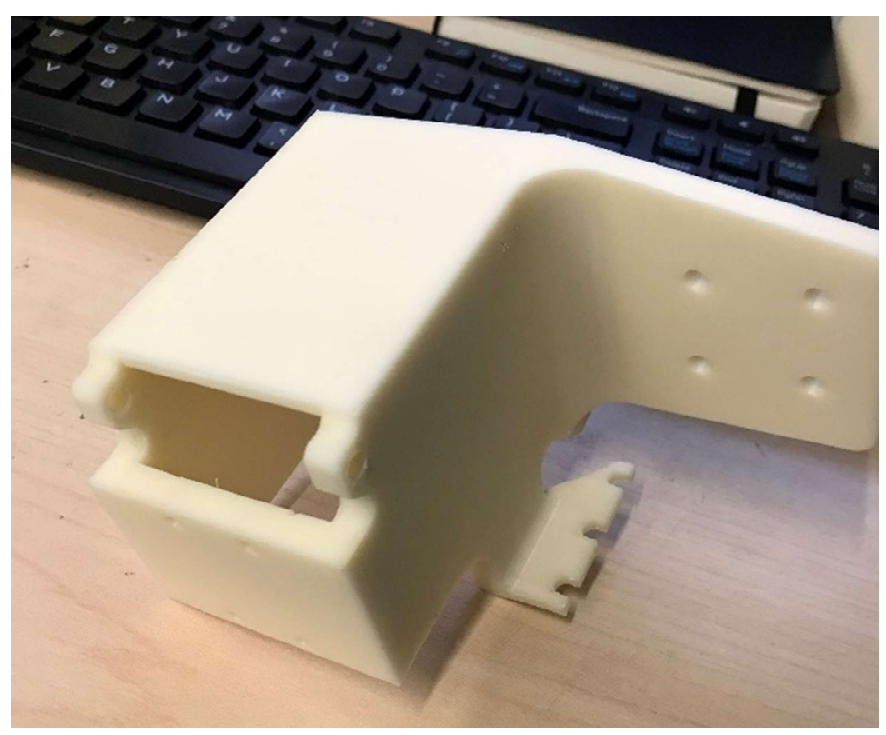

(B)

Figure 7.5 (A) CAD model of mount (b) 3D printed mount 


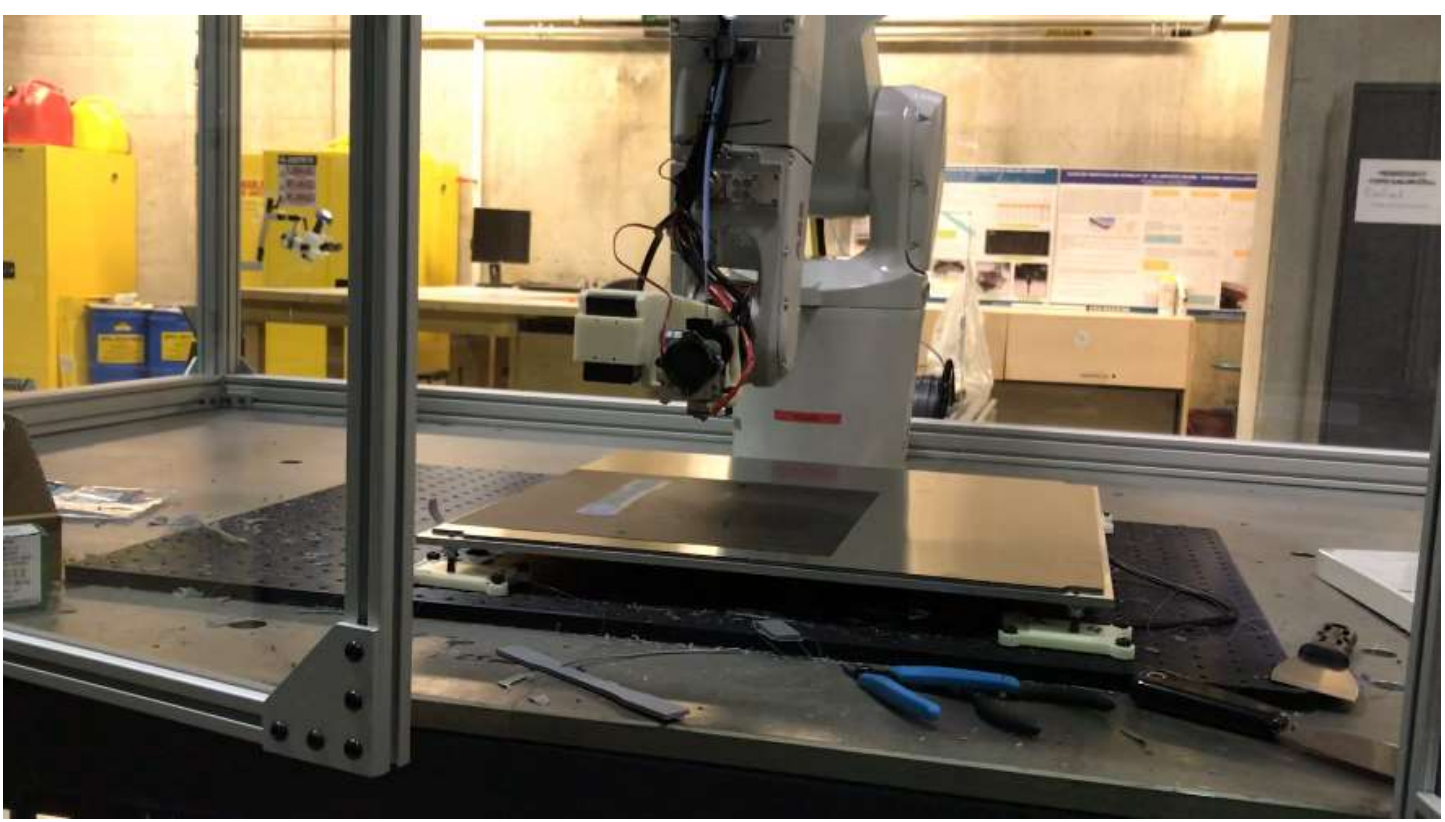

Figure 7.6 Laser and Hotend integrated to IRB1200

\subsection{ROBODK Software integration.}

The virtual work cell was setup with the CAD model as seen in Figure 7.7.The TCP for both the laser and the hot end were inserted into the ROBODK model. A D638 coupon was sliced in CURA and the resulting GCODE file was imported into ROBODK.

The post processor for ROBODK was modified to include an interrupt at each layer change on the coupon. This was done by looking for the layer change comment that is automatically generated by the slicing software, which in this case was CURA. The robot motion algorithm in ROBODK is written in Python and is a robot neutral environment with a generic output that can be post processed into each system's proprietary language, which in the case of ABB is RAPID. At the beginning of each layer change a function called SCAN is called, which will interrupt the current process and enter a sub procedure to scan the coupon. This process is repeated until the final layer is reached, at which the tool head is moved to a neutral position off the part and the robot is motion is completed. 


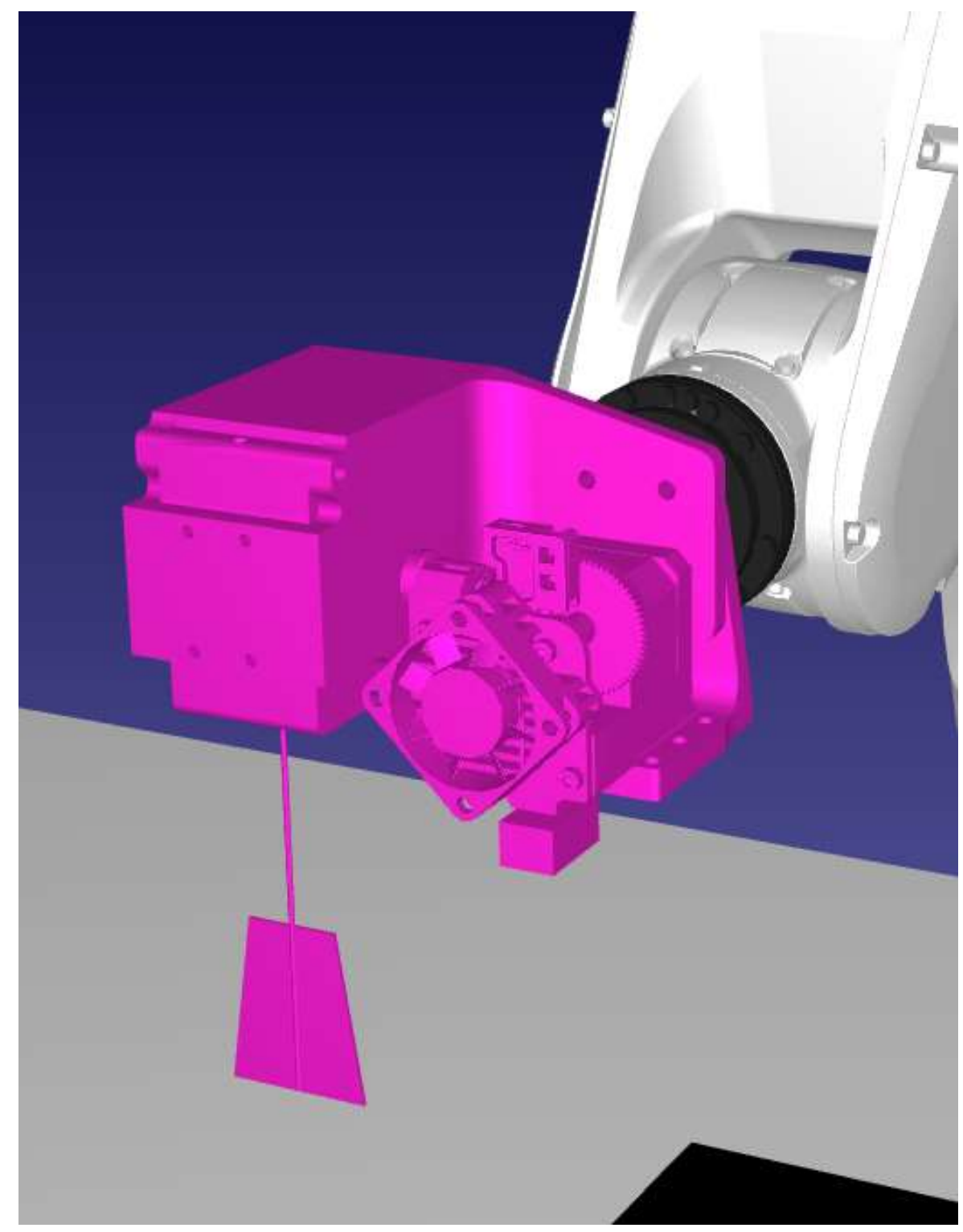

Figure 7.7 CAD Model of Scanner and Hotend integrated to IRB1200 Virtual Model 


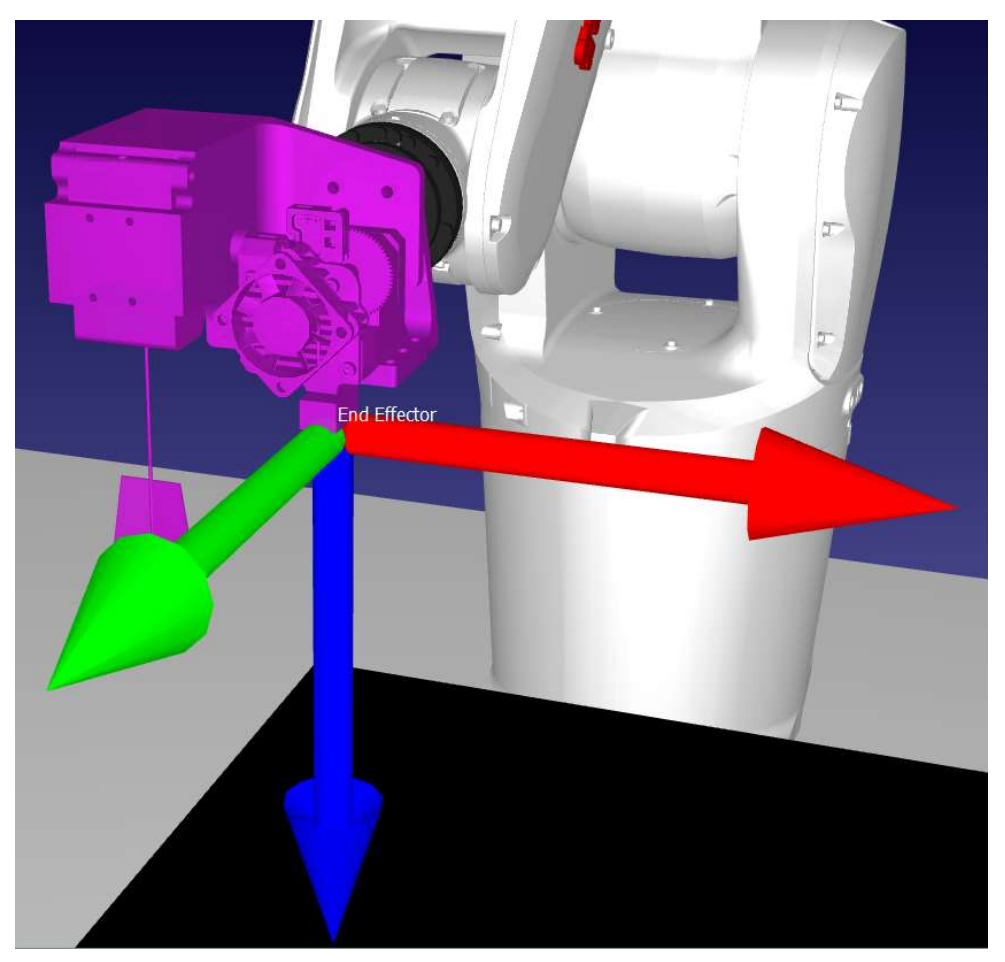

(A)

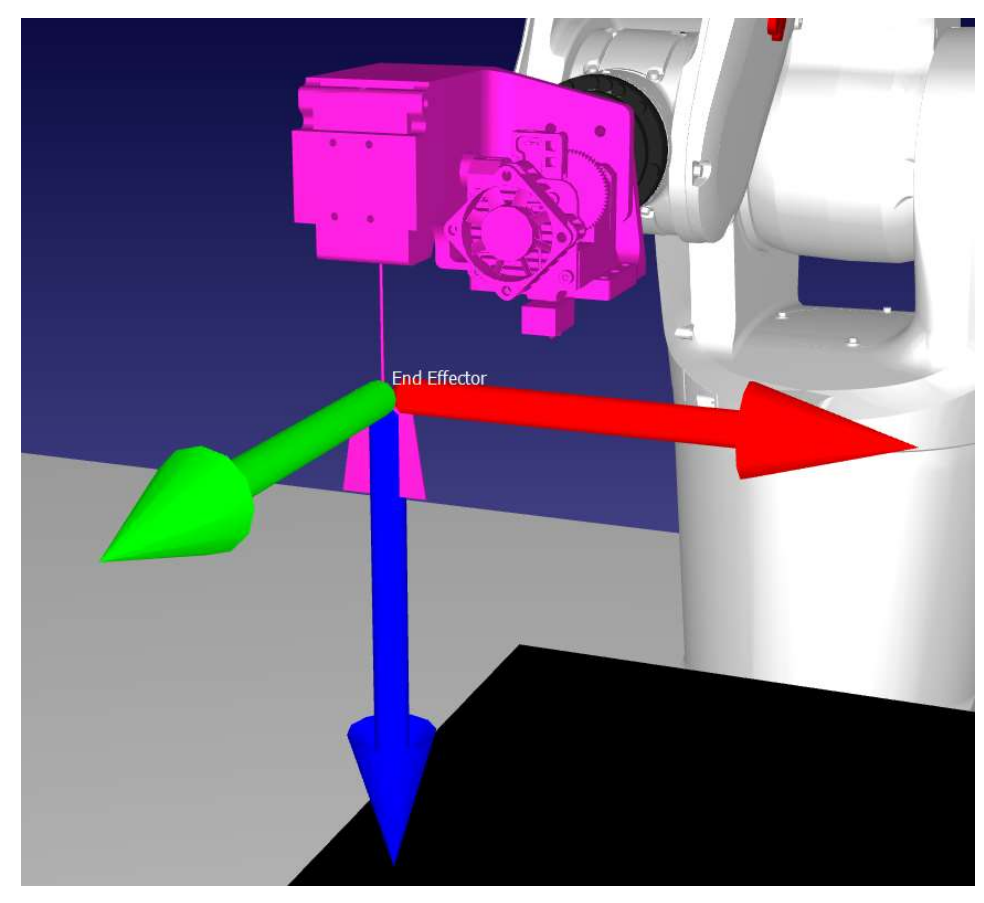

(B)

Figure 7.8(A) TCP for FFF nozzle, (B) TCP for LJ-V7080 Scanner 


\subsection{Scan subroutine}

The SCAN subroutine requires specifying the $x, y$ coordinaants of the point from which the scan begins as well as the $z$ level for the TCP of the scanner to be placed at. The laser head was triggered from the robot as the TCP moved through the trajectory. A parallel function called $T C P$ for communicating to the host computer is run simultaneously in the background. The TCP function sends data through the TCP/IP protocol over an Ethernet connection with the host computer.

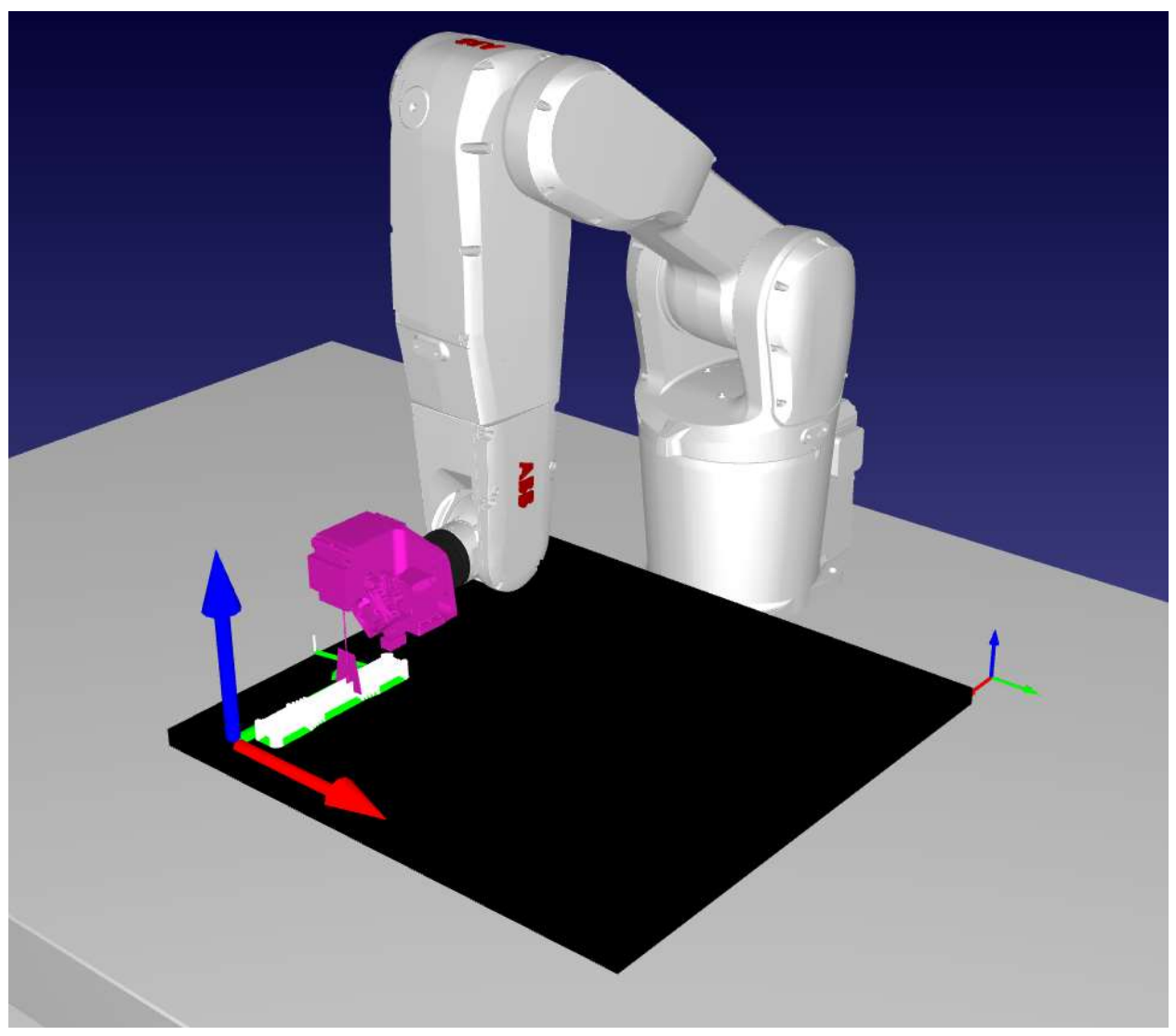

Figure 7.9 D638 Coupon undergoing an inspection 


\subsection{Keyence LJ-V7080 Control software}

Keyence provided $\mathrm{C}++$ libraries for interfacing with the laser controller to trigger the laser and receive data from the box. $\mathrm{A} \mathrm{C}++$ console program was written for retrieving the line data from the head and saving each line to a Comma Separated Value (CSV) file corresponding to the layer number. The console program also interfaced with the robot through the TCP/IP Ethernet communication protocol to for sending and receiving position data from the IRC5 controller.

\subsection{Scan Results}

The scan was completed and the results from the CSV file were post processed in MATLAB using the plotting toolbox. The scanning added an additional 12 minutes to the print time for a total fabrication time of 1 hour 20 minutes. The results were rendered for qualitative representation of the ASTM D638 Coupon. The ridges of the deposited filament are very clearly represented as seen in Figure 7.10. A false colour image can be seen in Figure 7.11, which clearly

shows the under extrusion at the end of the print and the definition of each bead. The following figure, Figure 0.12 , is a 3D representation of the first layer of the coupon, which shows the bead height and spacing as well as the roughness of the bed surface. The axis dimension in the figures are unitless.

For clearer representation, the bed is masked out by sampling out a scan of the print area and removing any data that is below the nominal layer height. Using this method, only the image of the coupon can be represented as seen in Figure 7.13 for various angles of the stack up. Furthermore, it should be noted that the scan results are inverted. Various defects can be picked up, such as the travel lines on the coupon and the extra material that is left behind when the tool head lifts up to begin the scanning procedure. 
(A)

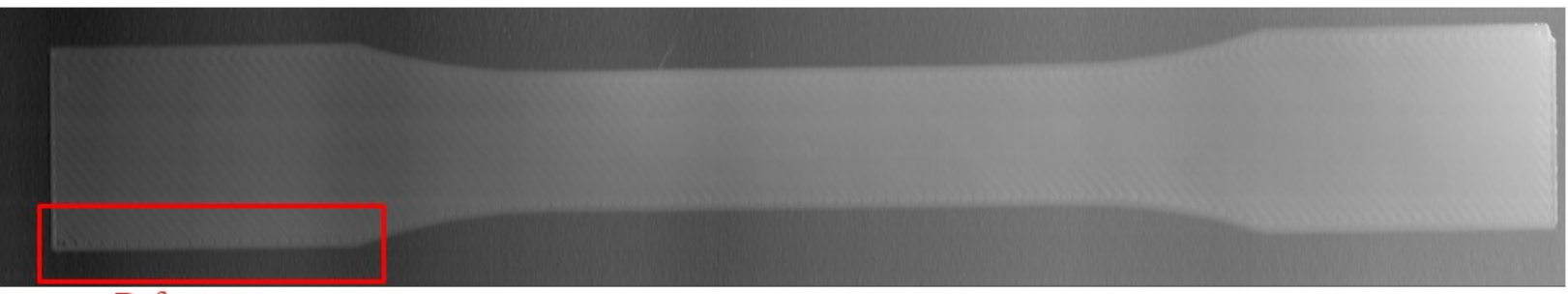

Defect area

(B)

Figure 7.10 (A) Photograph of D638 Coupon (B) Coupon scanned with LJ-7080

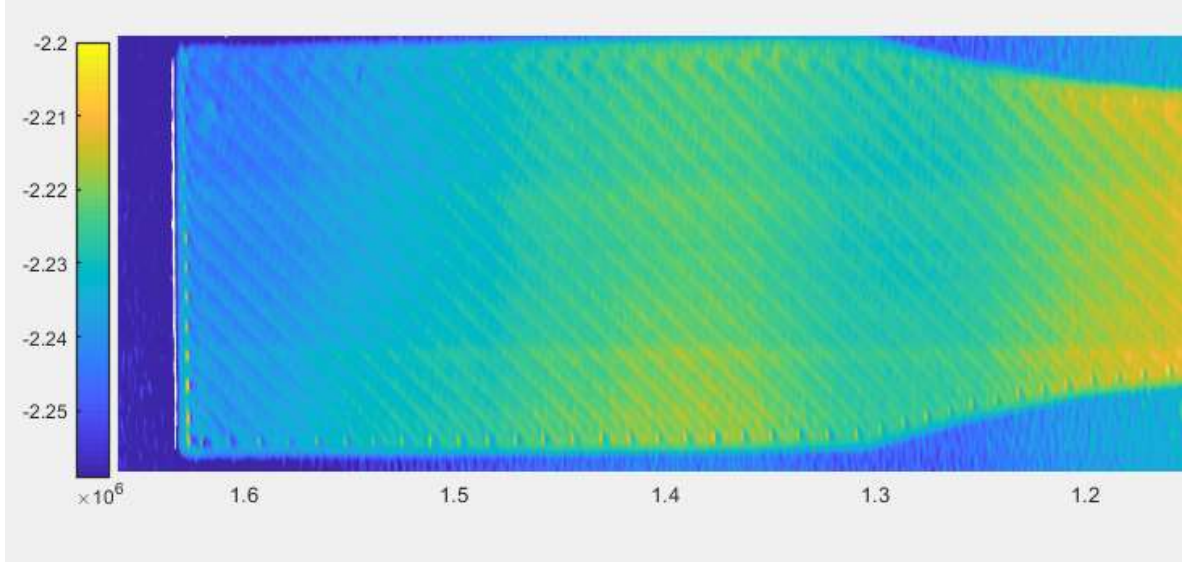

Figure 7.11 Zoomed in view of coupon scan highlighting defects with false colour

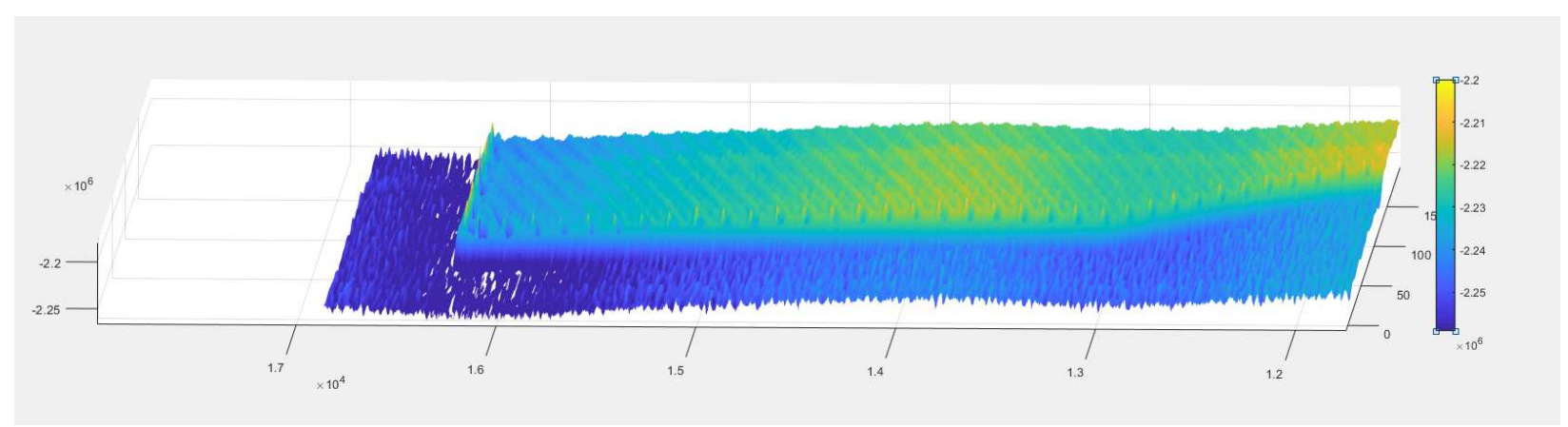

Figure $0.123 D$ representation of coupon 


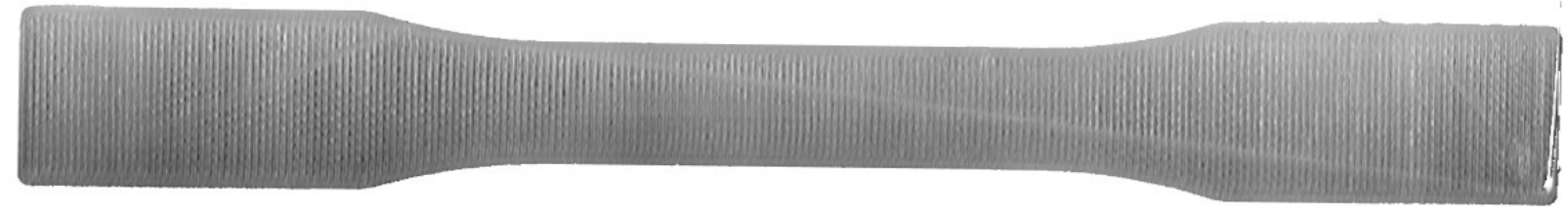

(A)

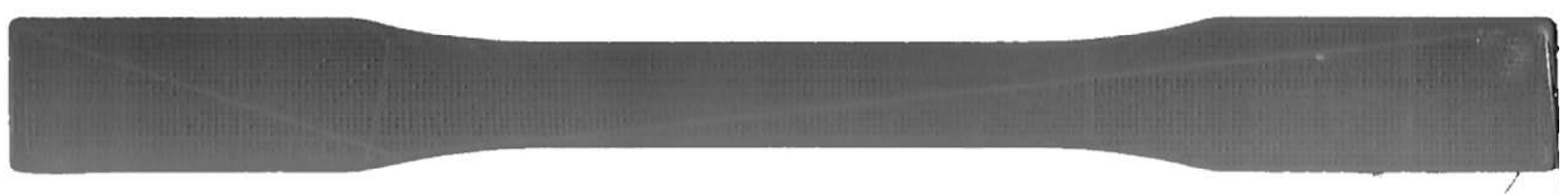

(B)

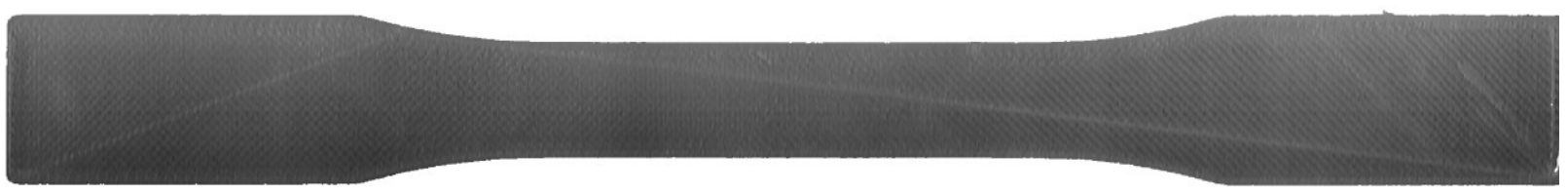

(C)

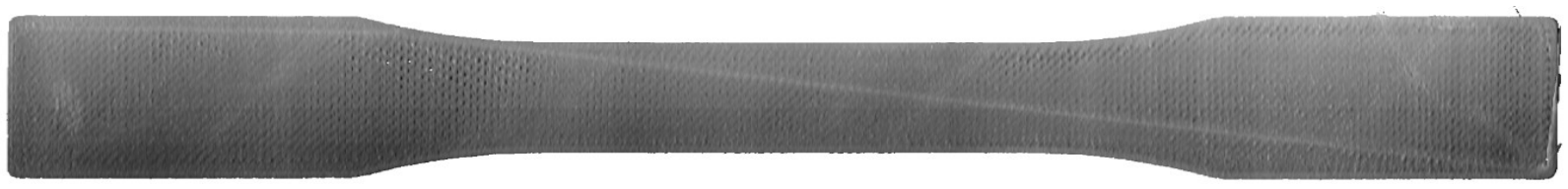

(D)

Figure 7.13 (A) O Degrees (B)90 Degrees (C) 45 Degrees (D) -45 degrees

\subsection{Conclusions}

The ASTM D638 coupon was successfully printed and inspected on a per layer basis. The complete scan for each layer required approximately 30 seconds to complete, for an additional 12 minutes to the total completion time for the coupon. For reference, the complete coupon without the inspection required approximately 1 hour and 20 minutes to complete. The relatively short scan time allows the process to be viable for use in a production environment.

The scan time can be reduced if other techniques for generating the robot targets and motion are used. The top speed for the IRB1200 is $7000 \mathrm{~mm} / \mathrm{s}$, but the speed was limited to $60 \mathrm{~mm} / \mathrm{s}$ for printing and due to constraints on the hardware $5 \mathrm{~mm} / \mathrm{s}$ for the scanning. Due to the very dense points for the scan, the motion controller becomes strained, continuously accelerate 
and decelerate to meet the small zone requirements to ensure an accurate scan. This issue can be solved by using a Fly-By-Points methodology outlined by ABB, where the TCP will continuously move through the points without stopping, while triggering the laser at that point. The technical reference manual from Keyence on the LJV7080 claims that the scanner is able to accurately pickup the line data on a moving surface. This methodology will have to be a future work as a software license for the IRC5 controller is required for this feature.

Currently the described methodology is satisfactory for the purposes of validating and inspecting parts manufactured by the CFF work cell. Future work will include implementing the defect detection algorithm and integrating the scanner onto a tool changer for more robust operation of the work cell. Furthermore, a stitching algorithm needs to be developed for parts that are not planar or larger than the scan line width.

A major concern with this in process inspection procedure is that it may induce defects while in the process of detecting defects. One such case was that the nozzle lifting up from the part surface left behind material on the part that built up through the layers. Similarly, the nozzle would cause a defect when returning to deposit material after the scanning procedure was completed. For future iterations of this methodology, it will be necessary to optimise the process parameters such that, the extra material is not left behind or added to the part when the scan procedure is completed. Furthermore, the scan time can contribute to defects by allowing the previous layer to cool down. Depending on the material this may reduce the inter layer bond strength. A solution to this may be a heated enclosure, however the LJ-V7080 has a maximum operating temperature of $+60^{\circ} \mathrm{C}$, which reduces the applications. 
Appendix A- Dimensioned Drawings for Gripper 


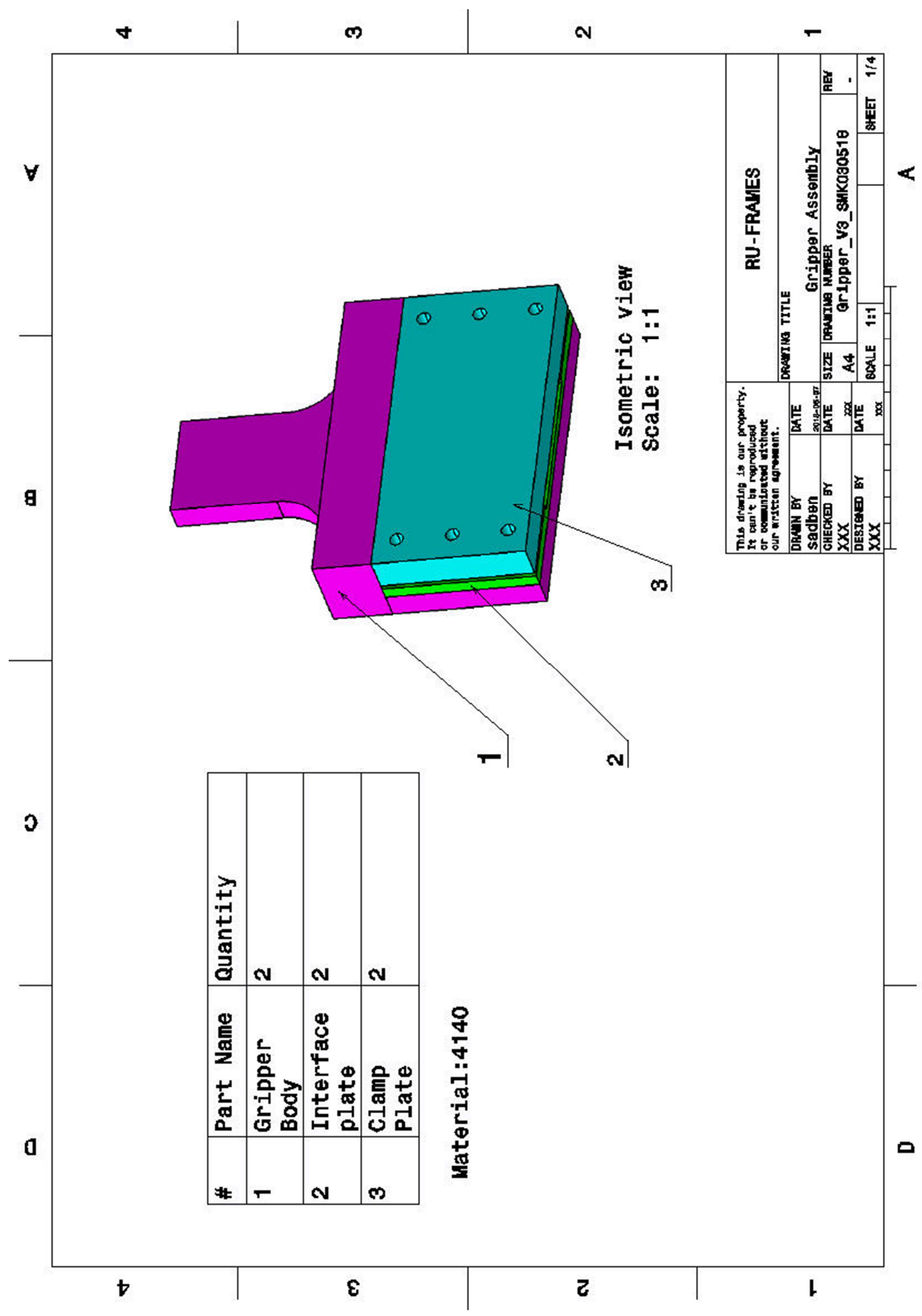




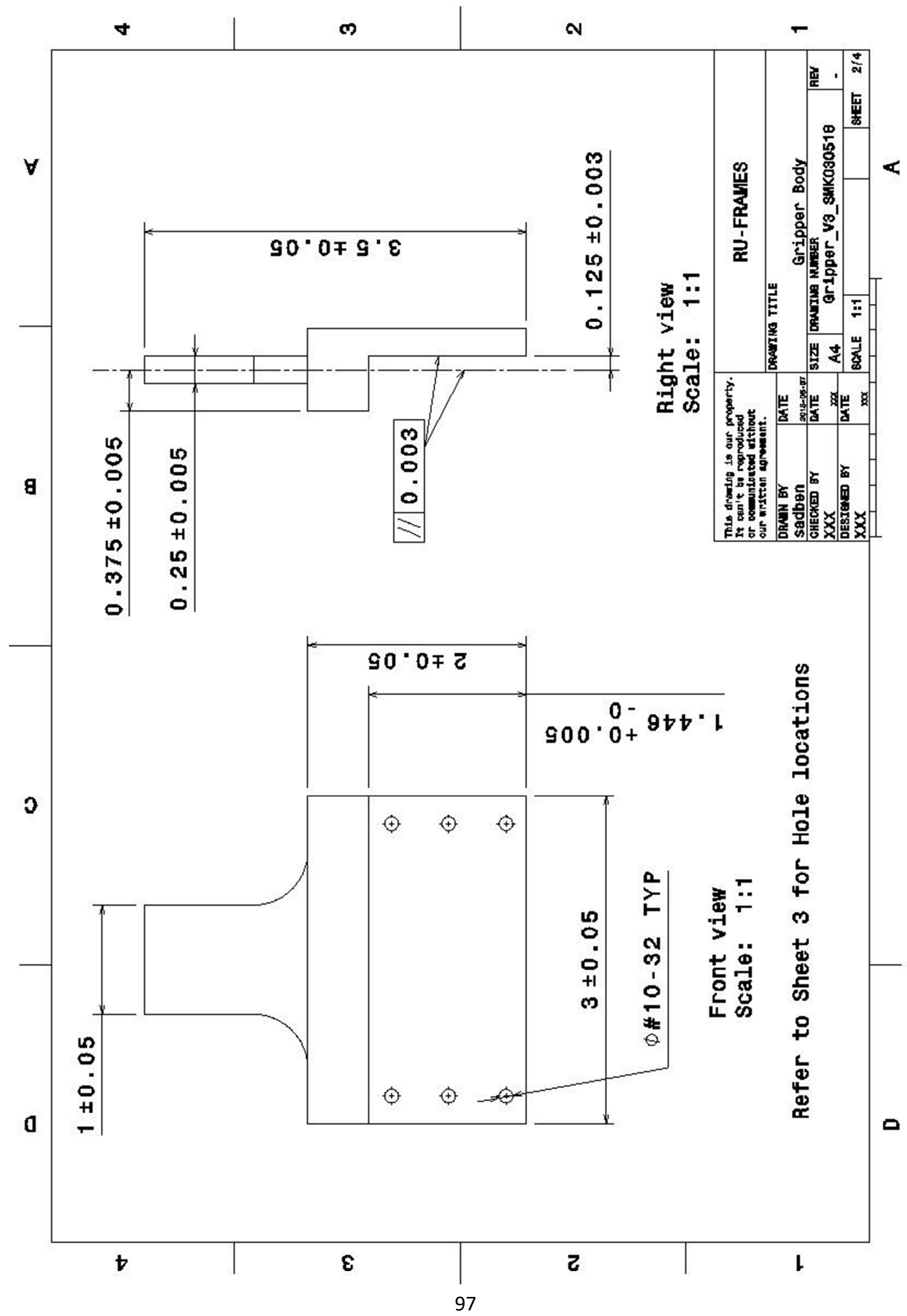




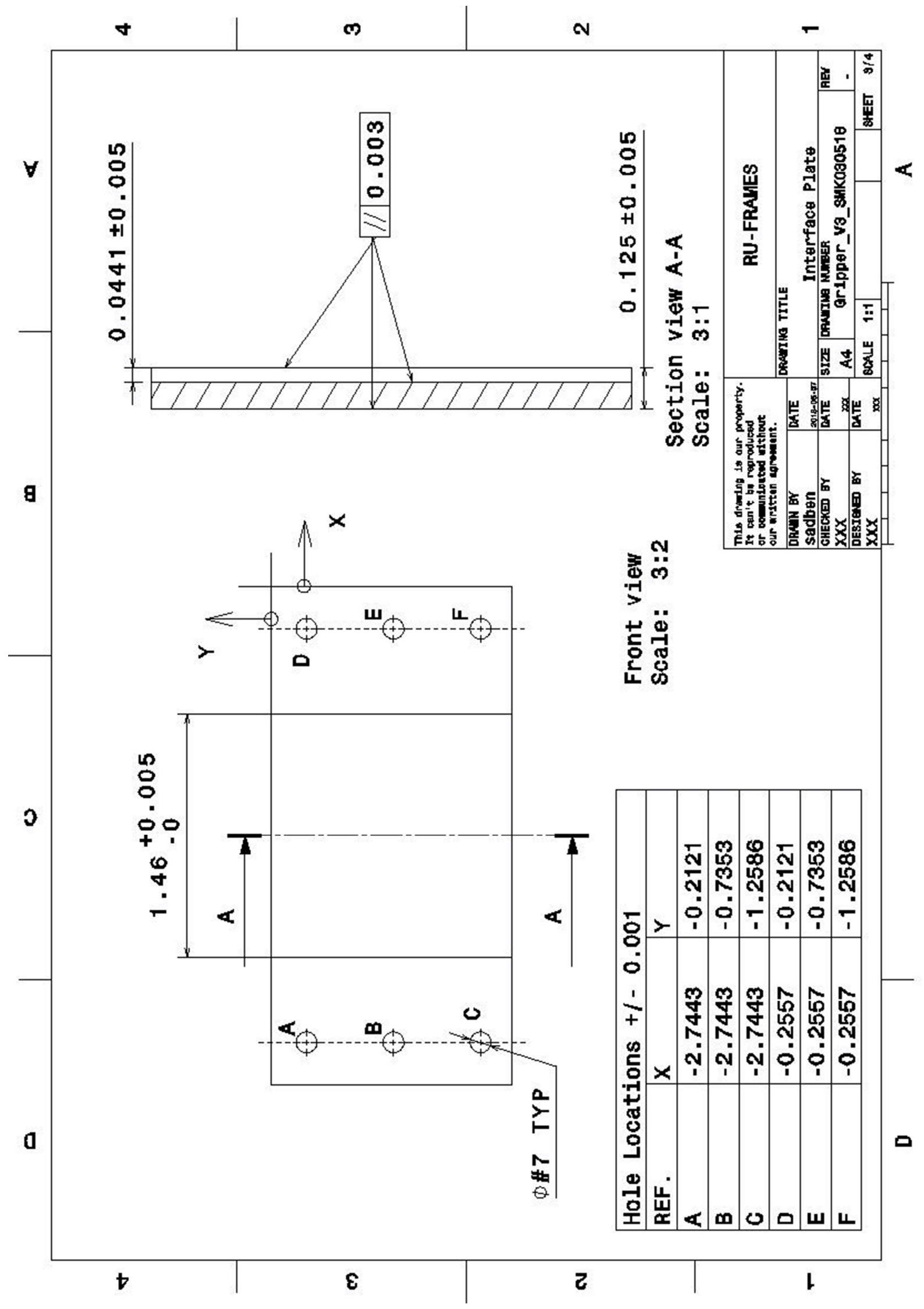




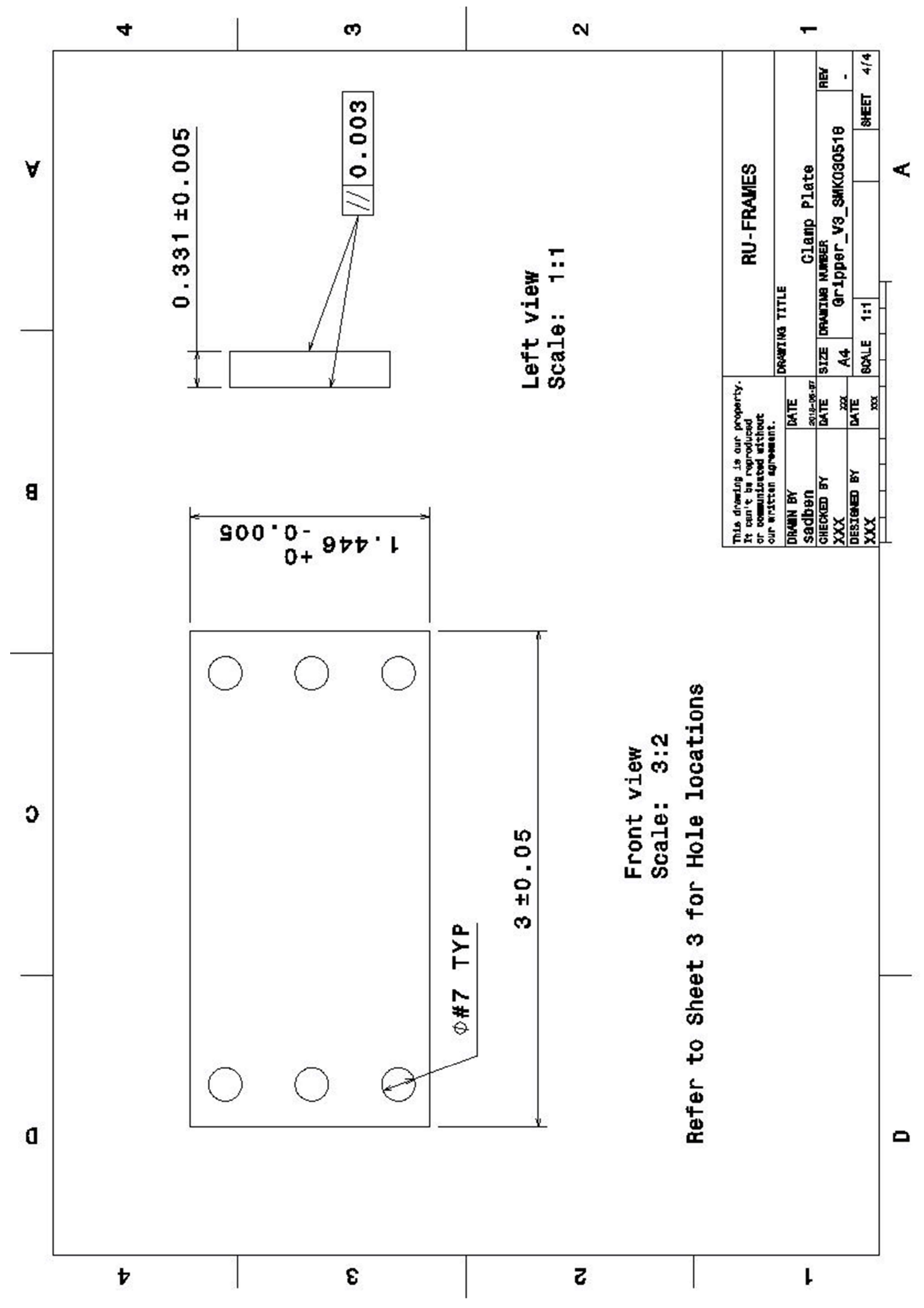


Appendix B- Dimensioned Drawings for 3D-Printing Head 


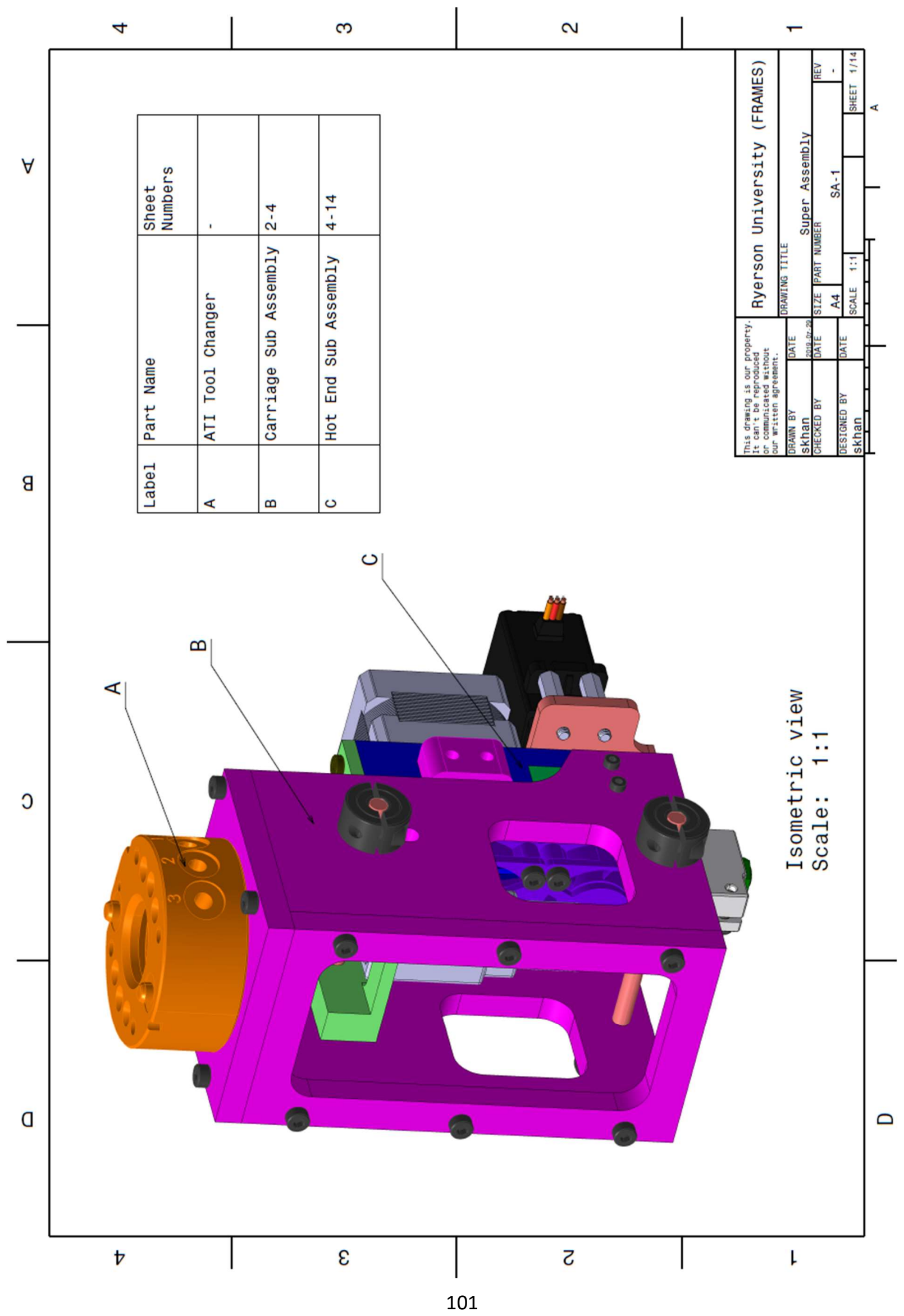




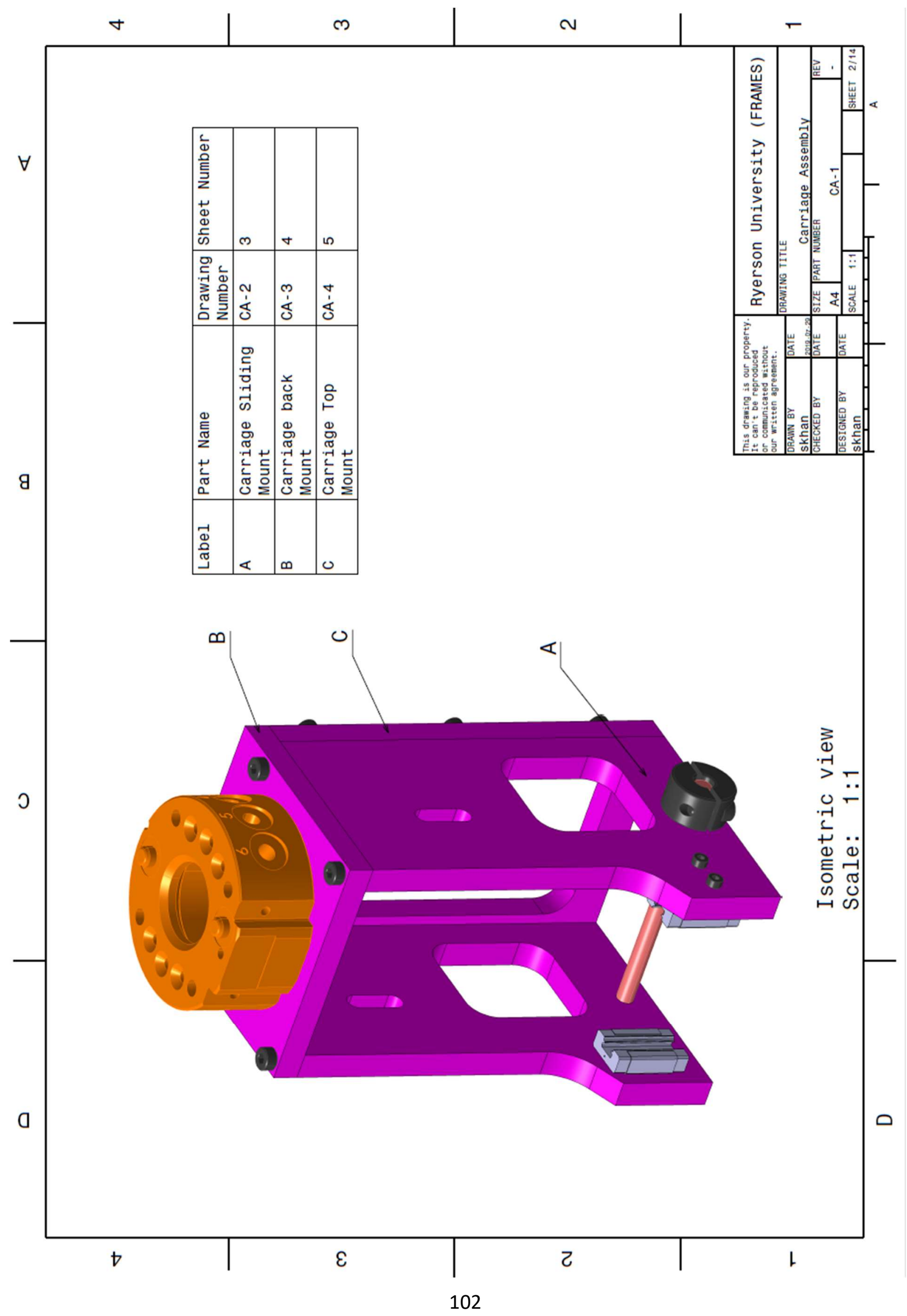




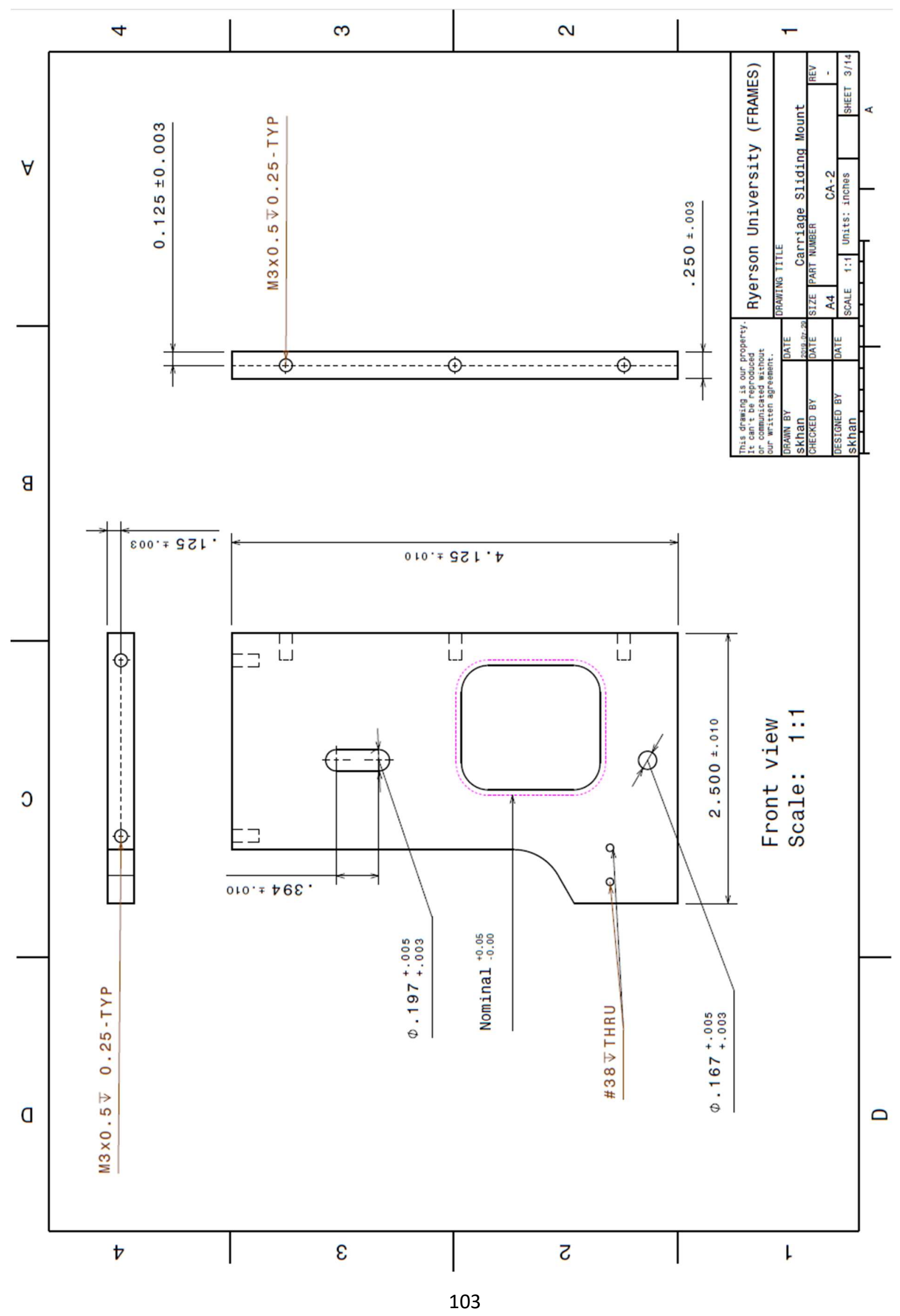




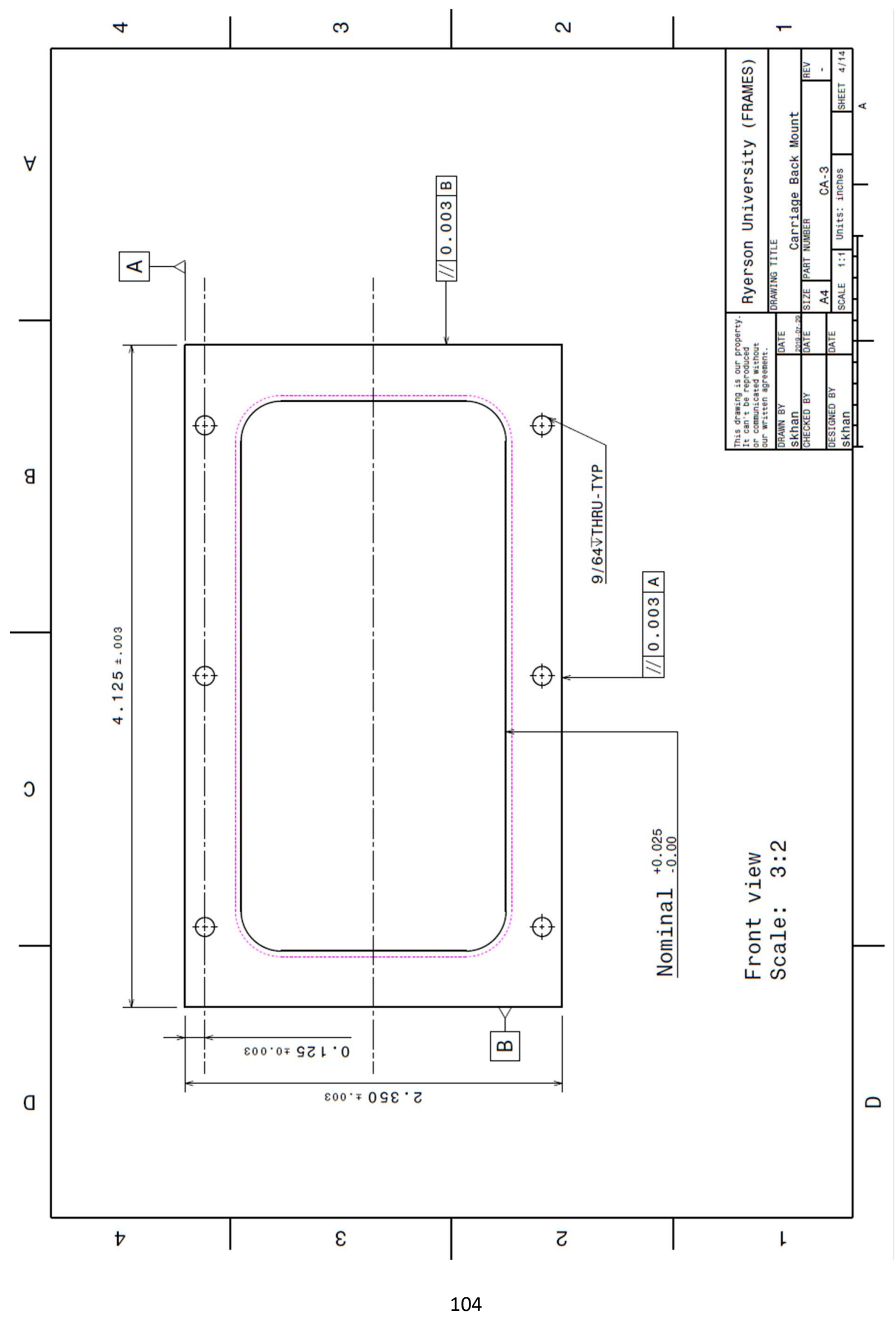




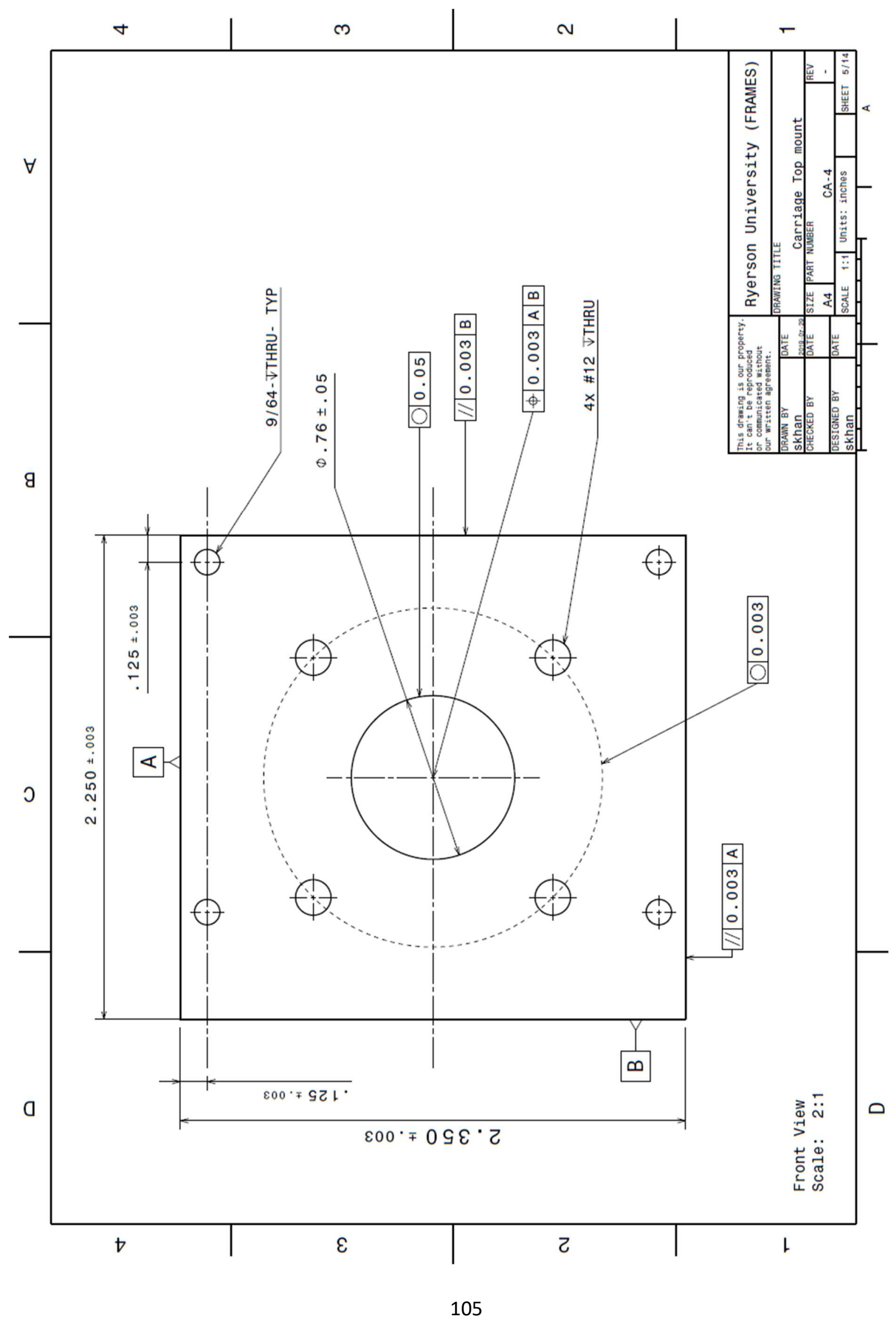




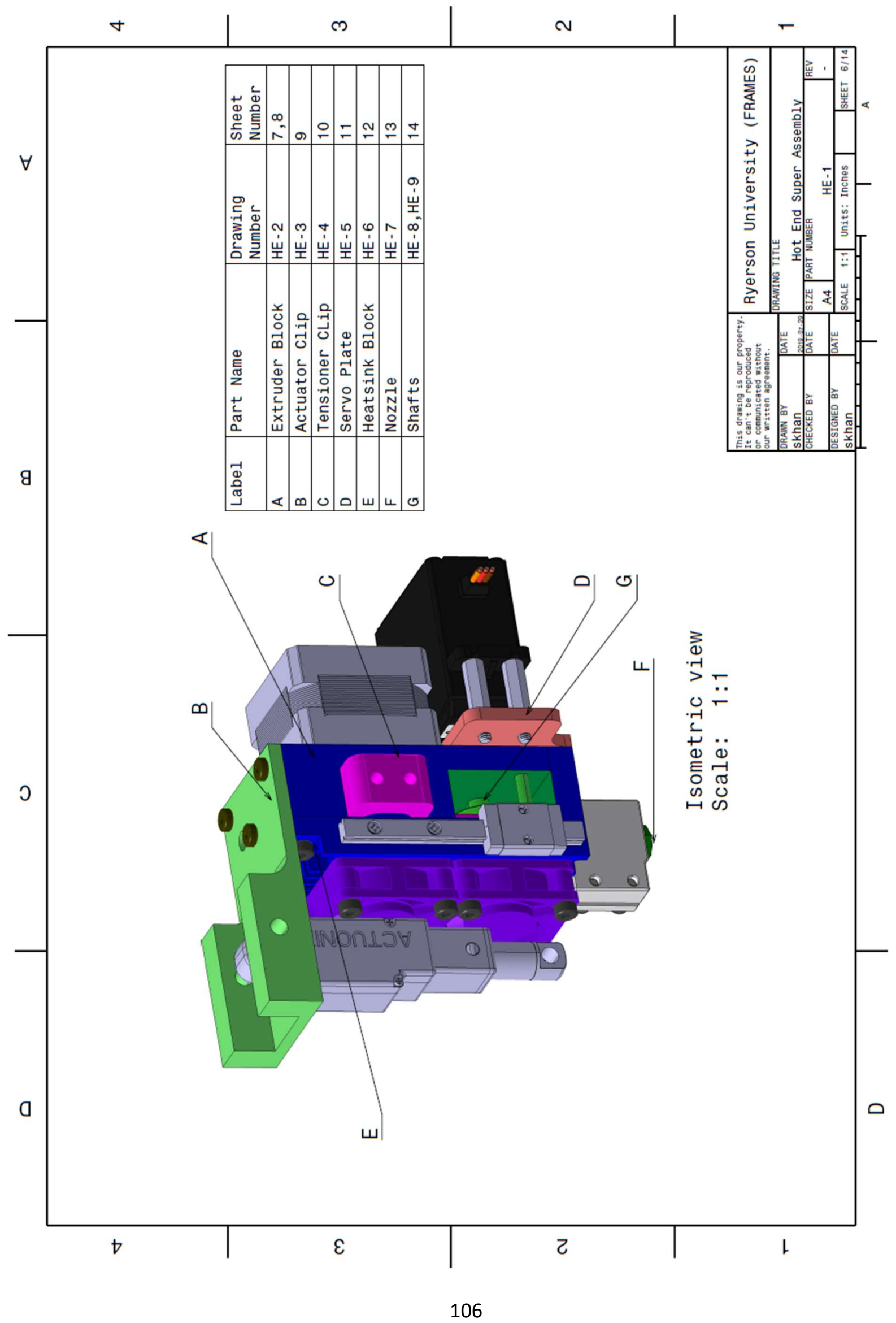



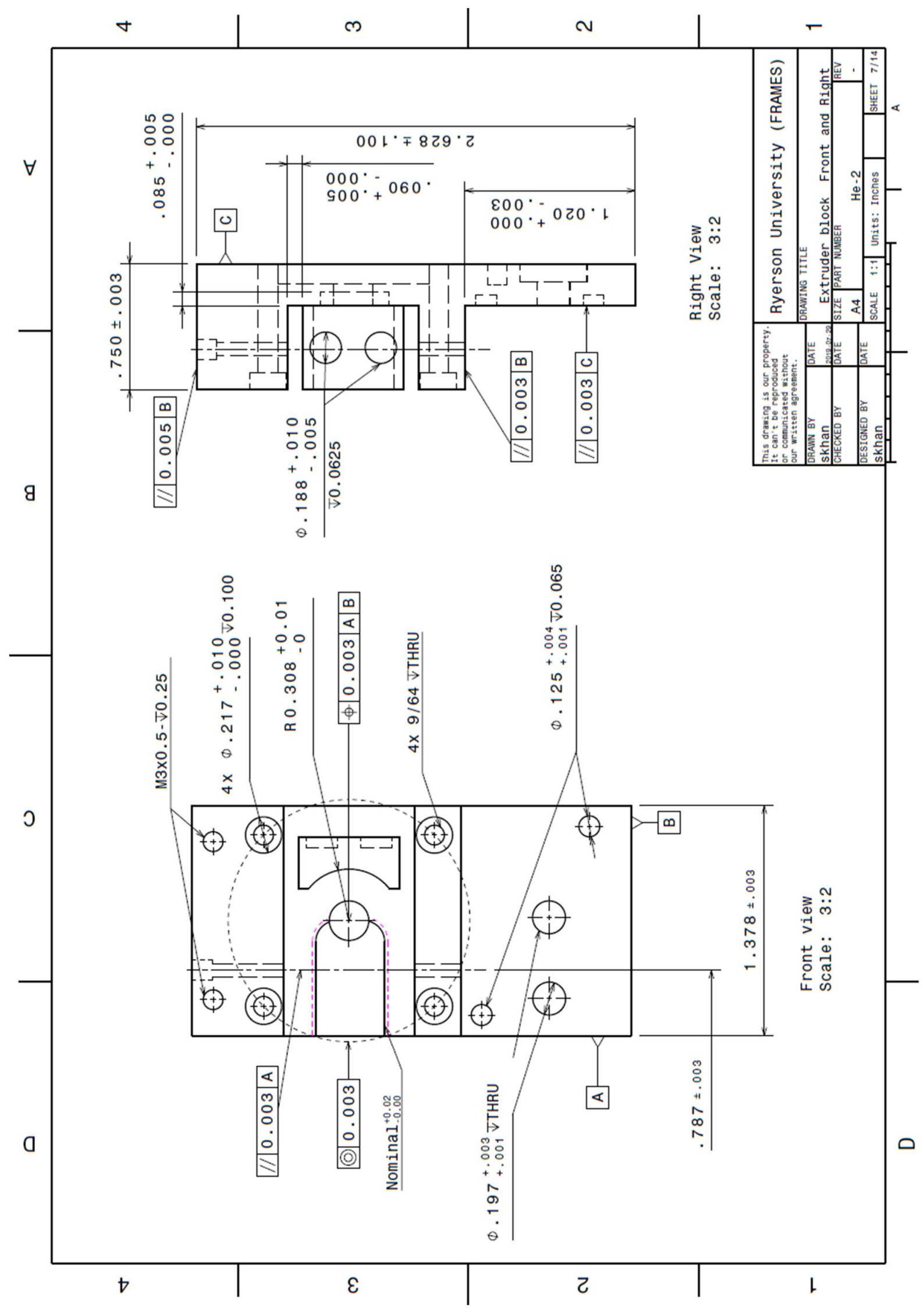


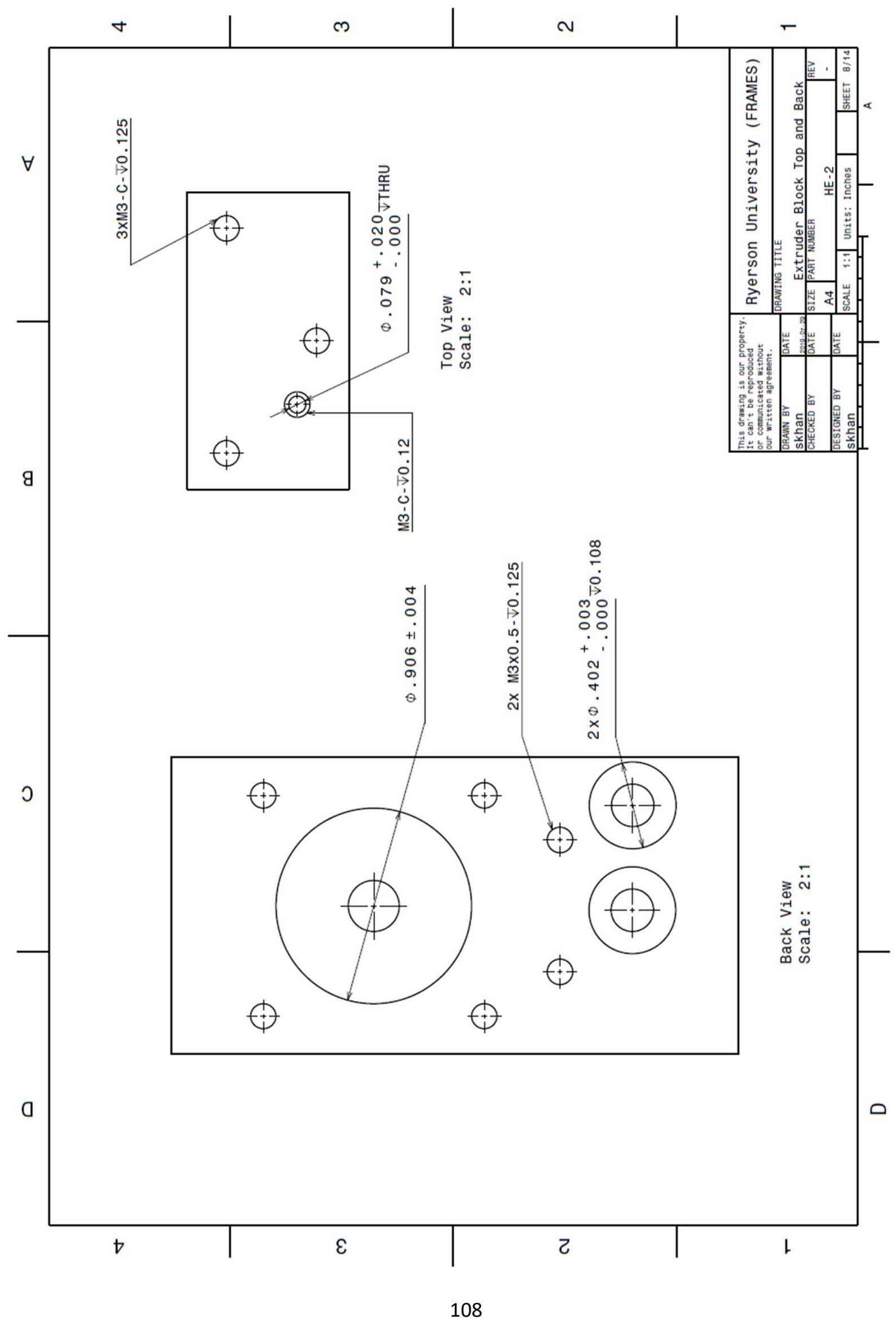




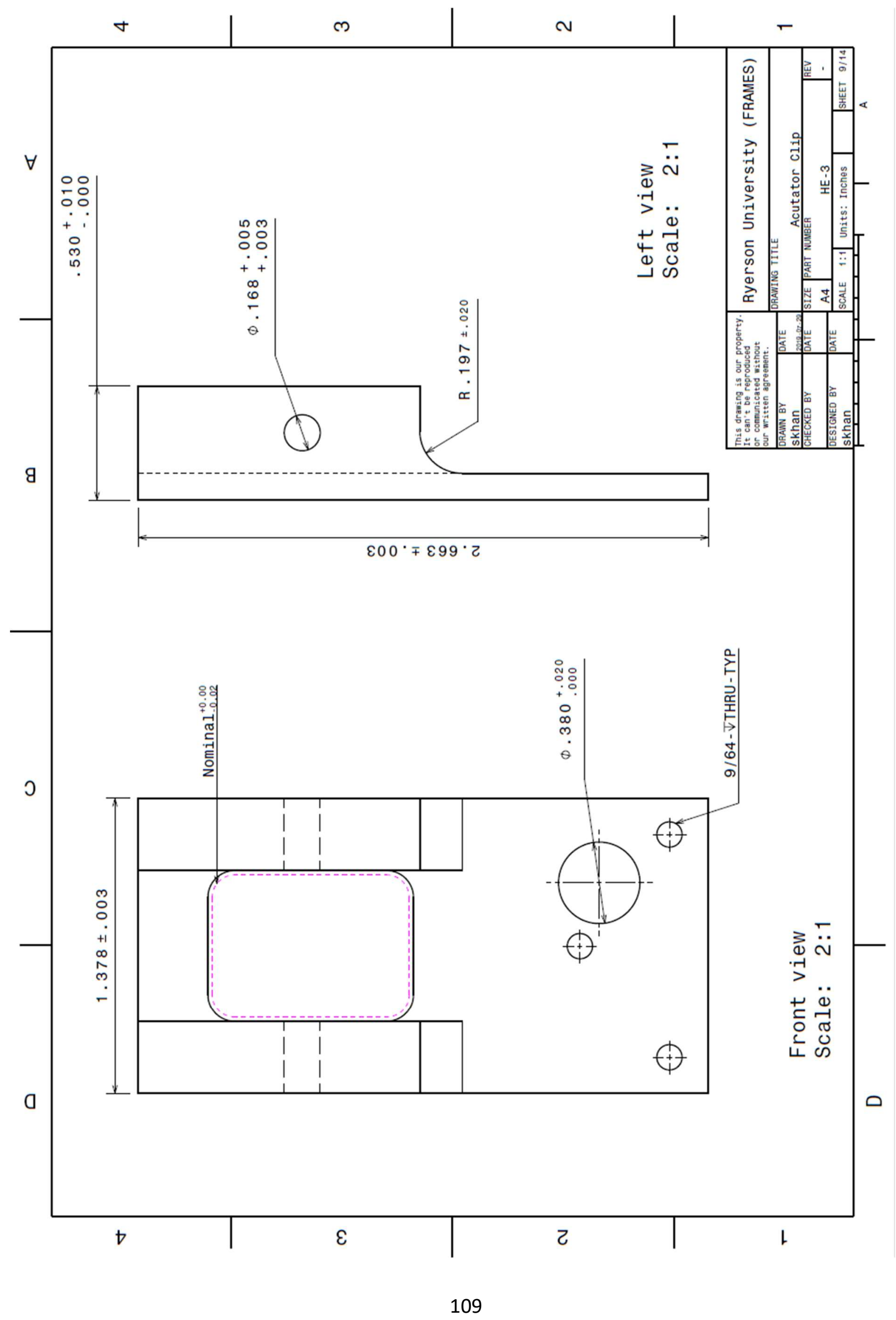




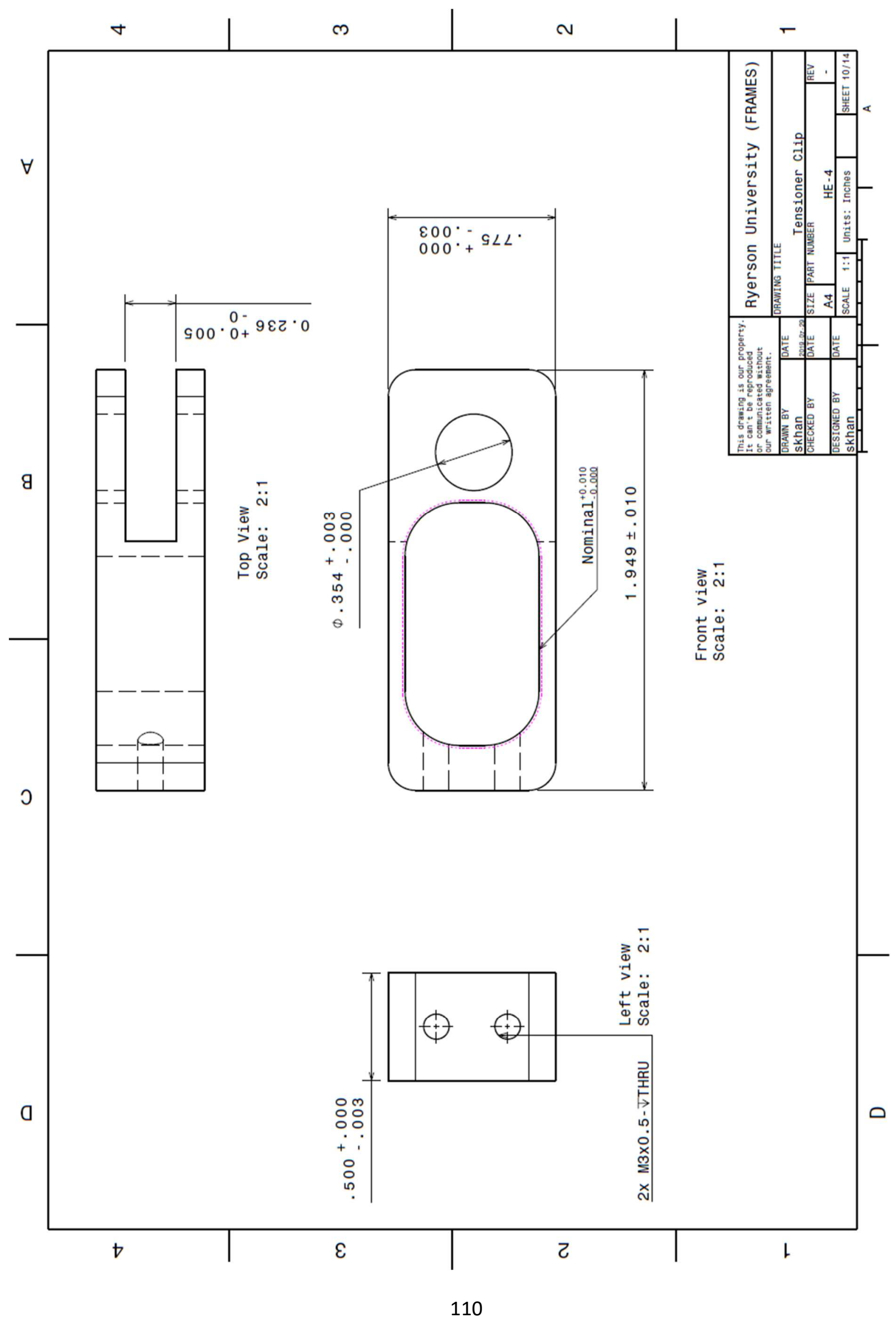




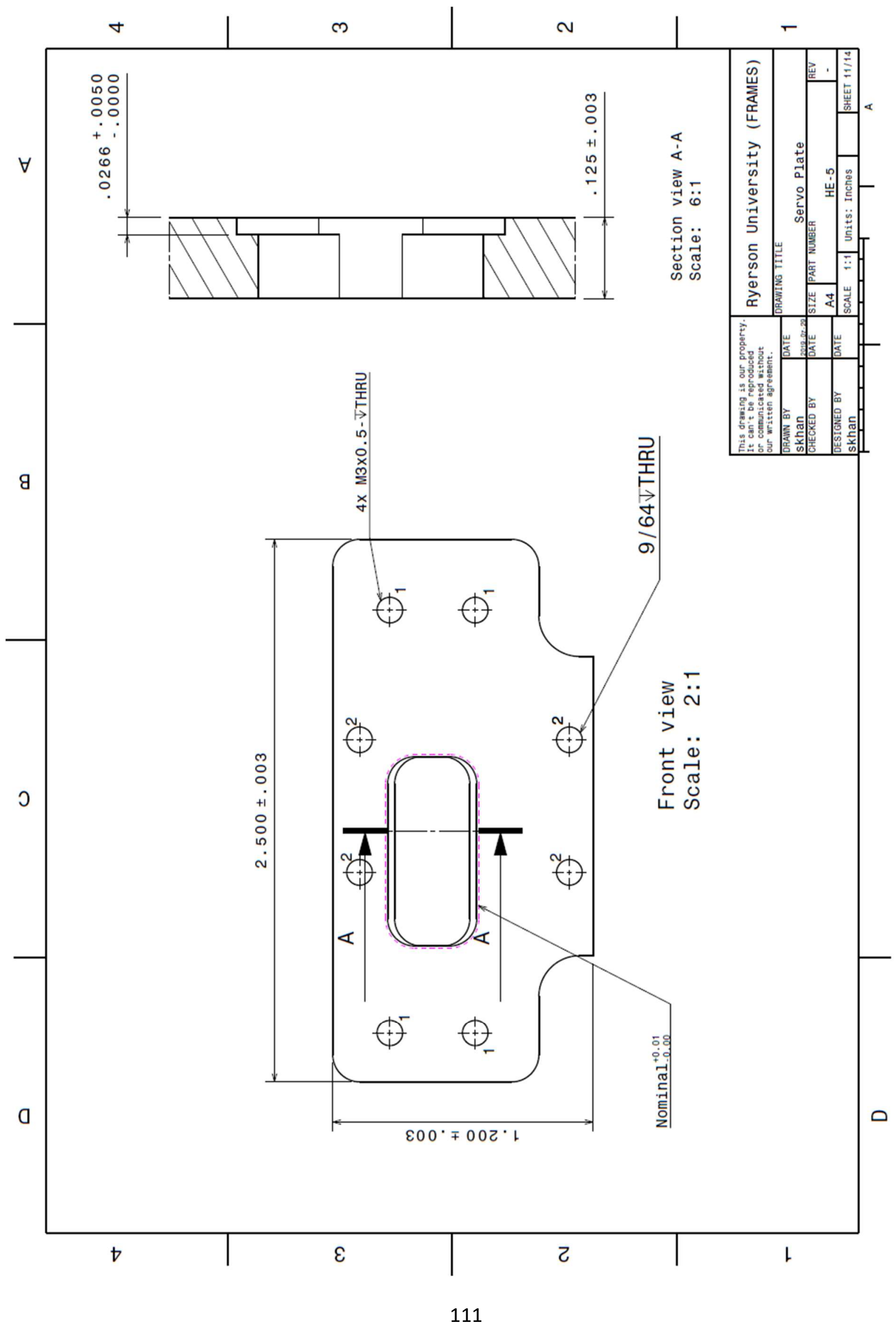




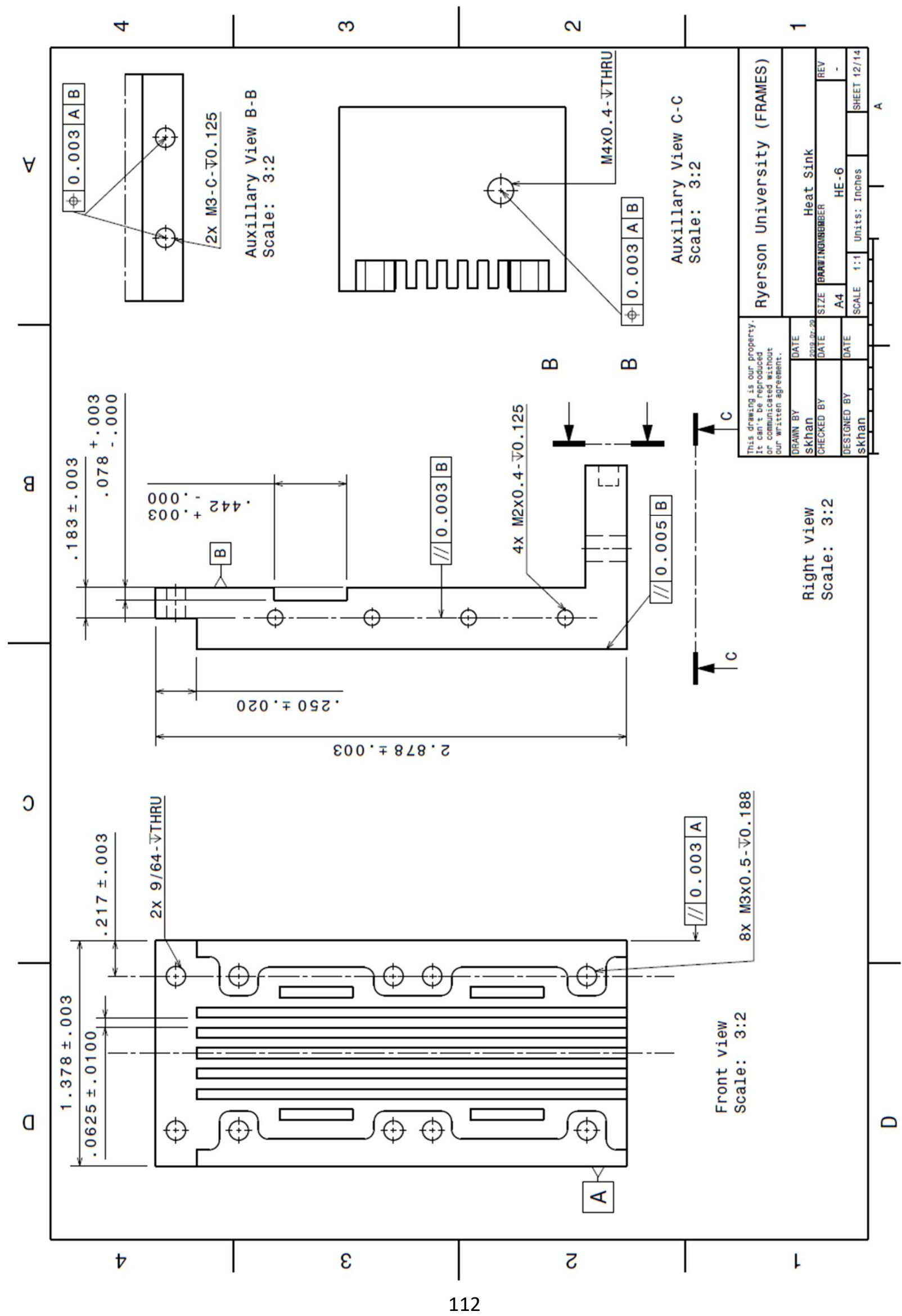




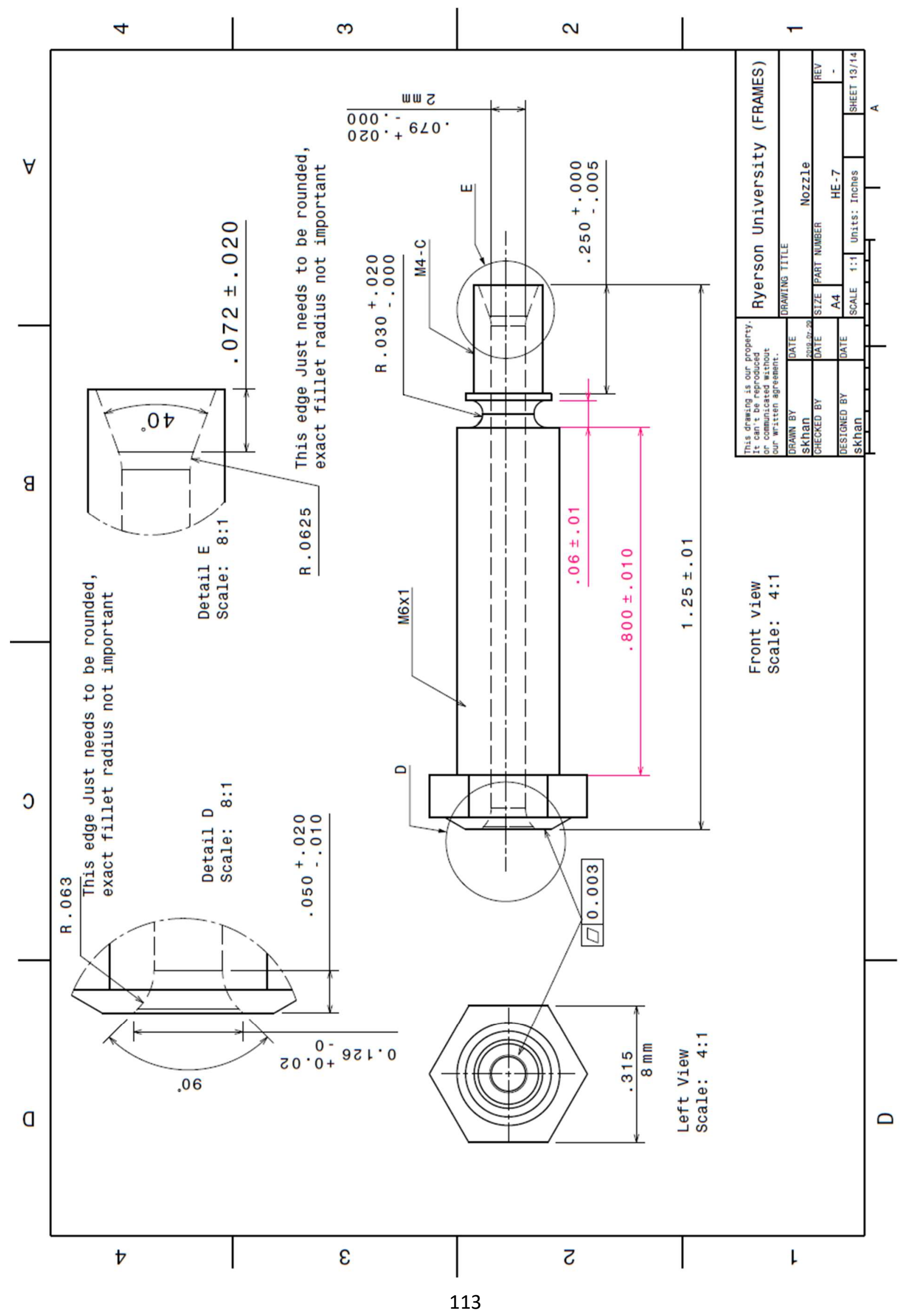




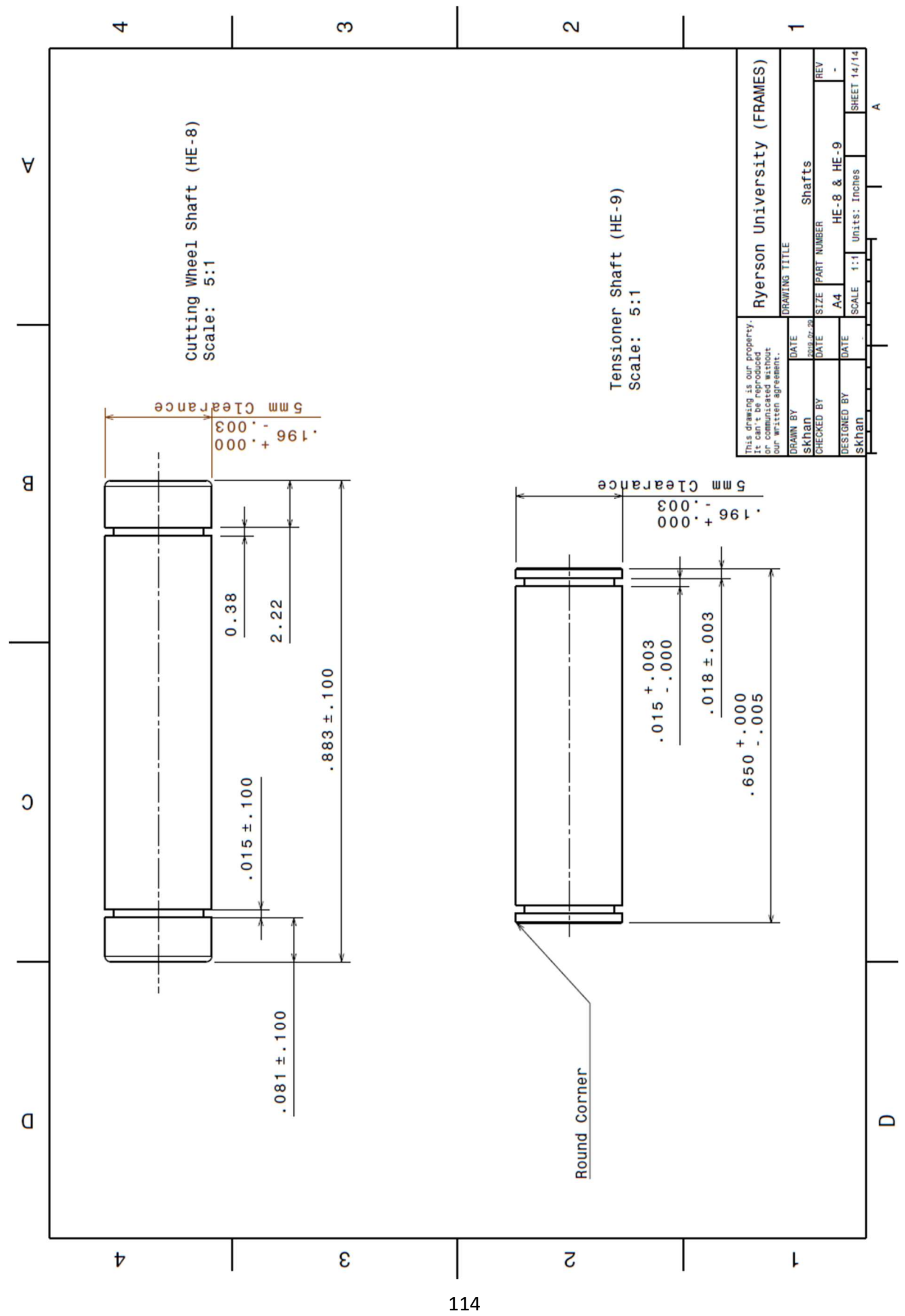




\section{$\underline{\text { References }}$}

[1] A. Baker and M. Scott, Composite Materials for Aircraft Structures, Reston: AIAA, 2016.

[2] H.-J. A. L. Dirk, C. Ward and K. D. Potter, "The engineering aspects of automated prepreg layup: History, present and future," Composites Part B: Engineering, vol. 43, pp. 997-1009, 2012.

[3] X. Olsthoorn, "Carbon dioxide emissions from international aviation: 1950--2050," Journal of Air Transport Management, vol. 7, pp. 87-93, 2001.

[4] B. E. Chitwood and M. S. Howeth, Apparatus for making laminated structural shapes by the controlled detrusive placement and polymerization of tectonic filamentous tapes, Google Patents, 1971.

[5] H. L. Eaton, "Cost effective tape laying," in 29th National SAMPE symposium. Reno, Nevada, USA, 1984.

[6] J. W. Saveriano, "Automated contour tape laying of composite materials," in National SAMPE Technical Conference, 1984.

[7] Stone and KL, "Automation in composite processing," in SAMPE symposium, Reno, Nevada, 1984.

[8] R. Meier, "An advanced control ystem for composite material placement," in SAMPE, Covina,California, 1986.

[9] B. T. Astrom, Manufacturing of polymer composites, Routledge, 2018.

[10] B. F. Tatting and Z. Gurdal, "Automated Finite Element Analysis of Elastically Tailored Plates," NASA, Blacksburg, Virginia, 2003.

[11] A. W. Blom, C. S. Lopes, P. J. Kromwijk, Z. Gurdal and P. P. Camanho, "A theoretical model to study the influence of tow-drop areas on the stiffness and strength of variable-stiffness laminates," Journal of composite materials, vol. 43, pp. 403-425, 2009.

[12] Z. Guerdal and R. Olmedo, "Composite laminates with spatially varying fiber orientations-'Variable stiffness panel concept'," in 33rd Structures, Structural Dynamics and Materials Conference, 1992.

[13] C. Waldhart, Z. Gurdal and C. Ribbens, "Analysis of tow placed, parallel fiber, variable stiffness laminates," in 37th Structure, Structural Dynamics and Materials Conference, 1996.

[14] D. C. Jegley, B. F. Tatting and Z. Gurdal, "Optimization of elastically tailored tow-placed plates with holes," AIAA, 2004.

[15] D. C. Jegley, B. F. Tatting and Z. Gurdal, "Tow-Steered Panels with Holes subjected to compression or shear loading," NASA, 2005. 
[16] K. C. Wu, J. D. Turpin, N. W. Gardner, B. K. Standford and R. A. Martin, "Structural Characteriazation of Advanced Composite Tow-Steered Shells with Large cutouts," in Structures, Structural Dynamics, and Materials Confrence, Kissimmee, Florida, 205.

[17] A. Alhajahmad, M. M. Abdalla and Z. Gurdal, "Optimal Design of a Pressurized Fuselage Panel with a Cutout using Tow-Placed Steered Fibers," in International Confrence on Engineering Optimization, Rio De Janeiro, 2008.

[18] S. Yau and T. Chou, "Strength of woven-fabric composites with drilled and molded holes," in Composite Materials: Testing and Design, 1988.

[19] M. Durante and A. Langella, "Bearing behaviour of Drilled and Molded-in Holes," Applied Composites Materials, pp. 297-306, 2009.

[20] M. W. Hyer and H. H. Lee, "The use of curvilinear fiber format to improve buckling resistance of composite plates with central circular holes," Composite structures, vol. 18, pp. 239-261, 1991.

[21] J. Huang and R. Haftka, "Optimization Design of Composite plates with holes for increased strength," Structural Multidisiplinary Optimization, vol. 30, pp. 334-341, 2005.

[22] C. S. Lopes, Z. Gurdal and P. P. Camanho, "Tailoring for strength of composite steered-fiber panels with cutouts," Composites, vol. 41, no. A, pp. 1760-1767, 2010.

[23] M. Montemurro and A. Catapano, "A general B-spline surfaces theoretical framework for optimisationf of variable angle-tow laminates," Composite Structures, vol. 209, pp. 561-578, 2019.

[24] P. A. Gustafson, "Fiber Tailoring for durability improvement of bolted composite plates," in Structures, Structural Dynamics and Materials Confrence, Denver, 2011.

[25] A. N. Dickson and D. P. Dowling, "Enhancing the bearing strength of woven carbon fibre thermoplastic composites through additive manufactuing," Composite Structures, vol. 212, pp. 381-388, 2019.

[26] L. G. Blok, M. L. Longana, H. Yu and B. K. S. Woods, "An investigation into 3D printing of fibre reinforced thermoplastic composites," Additive Manufacturing, vol. 22, pp. 176-186, 2018.

[27] H. Pruss and T. Vietor, "Design for Fiber-Reinforced Additive manufacturing," Journal of mechanical Design, vol. 137, 2015.

[28] X. Tian, T. Liu, C. Yang, Q. Wang and D. Li, "Interface and performance of 3D printed continuous carbon fiber reinforced PLA composites," Composites: Part A, vol. 88, pp. 198-205, 2016.

[29] Q. Hu, Y. Duan, H. Zhang, D. Liu, B. Yan and F. Peng, "Manufacturing and 3D printing of continuous carbon fiber prepreg filament," Journal of Material Science, vol. 53, pp. 1887-1898, 2018.

[30] M. Invernizzi, G. Natale, M. Levi, S. Turri and G. Griffini, "Uv Assisted 3D printing of glass and carbon fiberpreinforced Dual-cure Polymer Composites," Materials, vol. 9, p. 583, 2016. 
[31] W. De Backer, M. J. van Tooren and A. P. Berg, "Multi-Axis Multi-Material Fused Filament Fabrication with Continuous Fiber Reinforcement," in AIAA/ASCE/AHS/ASC STRUCTURES, STRUCTURAL DYNAMICS, AND MATERIALS CONFERENCE, Kissimmee, 2018.

[32] S. Liu, Y. Li and N. Li, "A novel free-hanging 3D printing method for continuous carbon fiber reinforced thermoplastic lattice truss core structures," Materials \& Design, vol. 137, pp. 235-244, 2018.

[33] M. Rüßmann, M. Lorenz, P. Gerbert, M. Waldner, J. Justus, P. Engel and M. Harnisch, "Industry 4.0: The future of productivity and growth in manufacturing industries," Boston Consulting Group, vol. 9, pp. 54-89, 2015.

[34] I. Campbell, D. Bourell and I. Gibson, "Additive manufacturing: rapid prototyping comes of age," Rapid prototyping journal, vol. 18, pp. 255-258, 2012.

[35] U. M. Dilberoglu, B. Gharehpapagh, U. Yaman and M. Dolen, "The role of additive manufacturing in the era of industry 4.0," Procedia Manufacturing, vol. 11, pp. 545-554, 2017.

[36] T. J. Hoskins, K. D. Dearn and S. N. Kukureka, "Mechanical performance of PEEK produced by additive manufacturing," Polymer Testing, vol. 70, pp. 511-519, 2018.

[37] T. D. Ngo, A. Kashani, G. Imbalzano, K. T. Q. Nguyen and D. Hui, "Additive manufacturing (3D printing): A review of materials, methods, applications and challenges," Composites Part B: Engineering, vol. 143, pp. 172-196, 2018.

[38] J. Steuben, D. L. Van Bossuyt and C. Turner, "Design for fused filament fabrication additive manufacturing," in ASME 2015 International Design Engineering Technical Conferences and Computers and Information in Engineering Conference, 2016.

[39] A. R. Torrado and D. A. Roberson, "Failure analysis and anisotropy evaluation of 3D-printed tensile test specimens of different geometries and print raster patterns," Journal of Failure Analysis and Prevention, vol. 16, pp. 154-164, 2016.

[40] M. Fernandez-Vicente, M. Canyada and A. Conejero, "Identifying limitations for design for manufacturing with desktop FFF 3D printers," International Journal of Rapid Manufacturing, vol. 5, pp. 116-128, 2015.

[41] B. Rankouhi, S. Javadpour, F. Delfanian and T. Letcher, "Failure analysis and mechanical characterization of 3D printed ABS with respect to layer thickness and orientation," Journal of Failure Analysis and Prevention, vol. 16, pp. 467-481, 2016.

[42] K. Fayazbakhsh, M. Movahedi and J. Kalman, "The impact of defects on tensile properties of 3D printed parts manufactured by fused filament fabrication," Materials Today Communications, vol. 18, pp. 140-148, 2019. 
[43] C. Koch, L. Van Hulle and N. Rudolph, "Investigation of mechanical anisotropy of the fused filament fabrication process via customized tool path generation," Additive Manufacturing, vol. 16, pp. 138145, 2017.

[44] Q. Y. Lu and C. H. Wong, "Applications of non-destructive testing techniques for post-process control of additively manufactured parts," Virtual and Physical Prototyping, vol. 12, pp. 301-321, 2017.

[45] V. K. Pathak, A. K. Singh, M. Sivadasan and N. K. Singh, "Framework for automated GD\&T inspection using 3D scanner," Journal of The Institution of Engineers (India): Series C, vol. 99, pp. 197-205, 2018.

[46] J. K. Na and E. K. Oneida, "Nondestructive evaluation method for standardization of fused filament fabrication based additive manufacturing," Additive Manufacturing, vol. 24, pp. 154-165, 2018.

[47] G. D. Goh, Y. L. Yap, H. K. J. Tan, S. L. Sing, G. L. Goh and W. Y. Yeong, "Process--Structure-Properties in Polymer Additive Manufacturing via Material Extrusion: A Review," Critical Reviews in Solid State and Materials Sciences, pp. 1-21, 2019.

[48] Q. Y. Lu and C. H. Wong, "Additive manufacturing process monitoring and control by nondestructive testing techniques: challenges and in-process monitoring," Virtual and physical prototyping, vol. 13, pp. 39-48, 2018.

[49] J. Straub, "Initial work on the characterization of additive manufacturing (3D printing) using software image analysis," Machines, vol. 3, pp. 55-71, 2015.

[50] J. Straub, "An approach to detecting deliberately introduced defects and micro-defects in 3D printed objects," in Pattern Recognition and Tracking XXVIII, 2017.

[51] J. Straub, "Characterization of 3D printing output using an optical sensing system," in Dimensional Optical Metrology and Inspection for Practical Applications IV, 2015.

[52] A. Ceruti, A. Liverani and T. Bombardi, "Augmented vision and interactive monitoring in 3D printing process," International Journal on Interactive Design and Manufacturing (IJIDeM), vol. 11, pp. 385395, 2017.

[53] M. Wu, V. V. Phoha, Y. B. Moon and A. K. Belman, "Detecting malicious defects in 3d printing process using machine learning and image classification," in ASME 2016 International Mechanical Engineering Congress and Exposition, 2016.

[54] I. Cummings, E. Hillstrom, R. Newton, E. Flynn and A. Wachtor, "In-process ultrasonic inspection of additive manufactured parts," in Topics in Modal Analysis \& Testing, Volume 10, Springer, 2016, pp. 235-247. 
[55] J. L. Horijon, W. D. Amstel, F. C. M. Couweleers and W. C. A. Ligthart, "Optical system of an industrial 3D laser scanner for solder paste inspection," in Three-Dimensional and Unconventional Imaging for Industrial Inspection and Metrology, 1996.

[56] K. Fayazbakhsh, M. A. Nik, D. Pasini and L. Lessard, "Defect layer method to capture effect of gaps and overlaps in variable stiffness laminates made by automated fiber placement," Composite Structures, vol. 97, pp. 245-251, 2013.

[57] Y. W.C, Roark's formulas for stress and straing, New York: McGraw-Hill, 2002.

[58] H. Ghiasi and e. al, "Optimum stacking squence design of composite materials part II: Variable stiffness Design," Composite Structures, vol. 93, no. 1, pp. 217-231, 2010.

[59] H. Ghiasi, D. Pasini and L. Lessar, "Optimum Stacking Sequence Design of Composite Materials Part I: Constant Stiffness Design," Composite Structures, vol. 90, no. 1, pp. 1-1, 2009.

[60] J. Park and e. al, "Stacking swquence design of ocmposite laminates for maximum strength using genetic algorithms," Composite Structures, vol. 52, pp. 217-231, 2001.

[61] S. Nagendra, R. Haftka and Z. Gurdal, "Genetic Algorithms for the desing of composite panels," Adganced Technology for Design and Fabrication of Composite Materials, pp. 193-143, 1995.

[62] M. Arian Nik and e. al, "A comparative study of the metamodeling methods for the design optimization of variable stiffness composites," Composite Structures, vol. 107, pp. 494-501, 2014.

[63] Keyence, "Keyence Highspeed 2D/3D Laser Profiler," 4th September 2019. [Online]. [Accessed 2019].

[64] ASTM, "Standard Test Method for Tensile Properties of Plastics," ASTM, West Conshohocken, 2019.

[65] S. Khan, K. Fayazbaksh, Z. Fawaz and M. A. Nik, "Curvilinear variable stiffness 3D printing technology for improved open-hole tensile strength," Additive Manufacturing, vol. 24, pp. 378385, 2018.

[66] E. Hardesty, W. Goldsworthy and H. Karlson, Geodesic path length compensator for compositetape placement head, Google Patents, 1974. 